
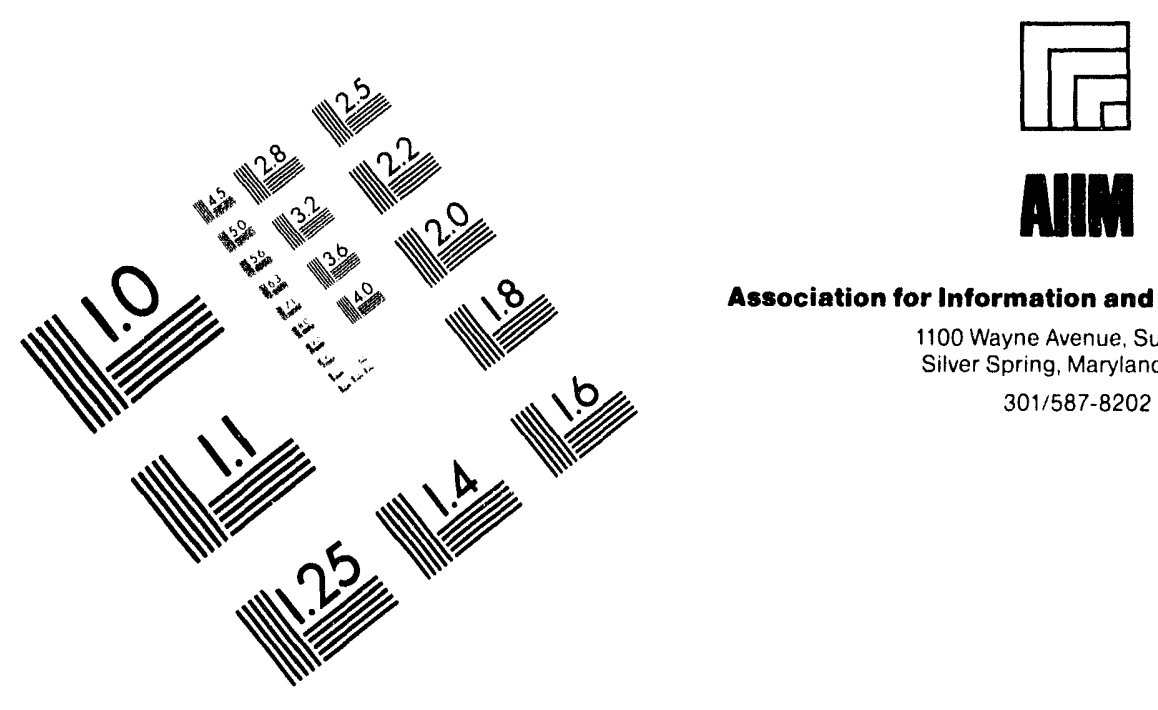

Association for Information and Image Management 1100 Wayne Avenue. Suite 1100 Silver Spring, Maryland 20910 301/587-8202

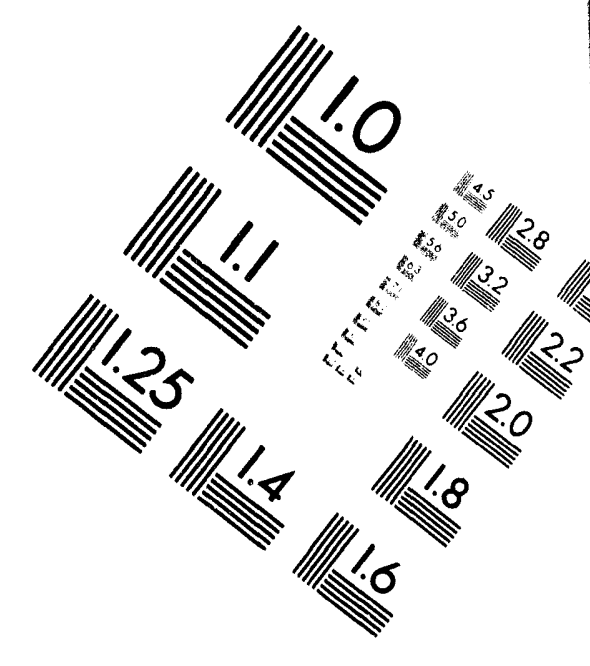

\title{
Centimeter
}

1
2 Inches
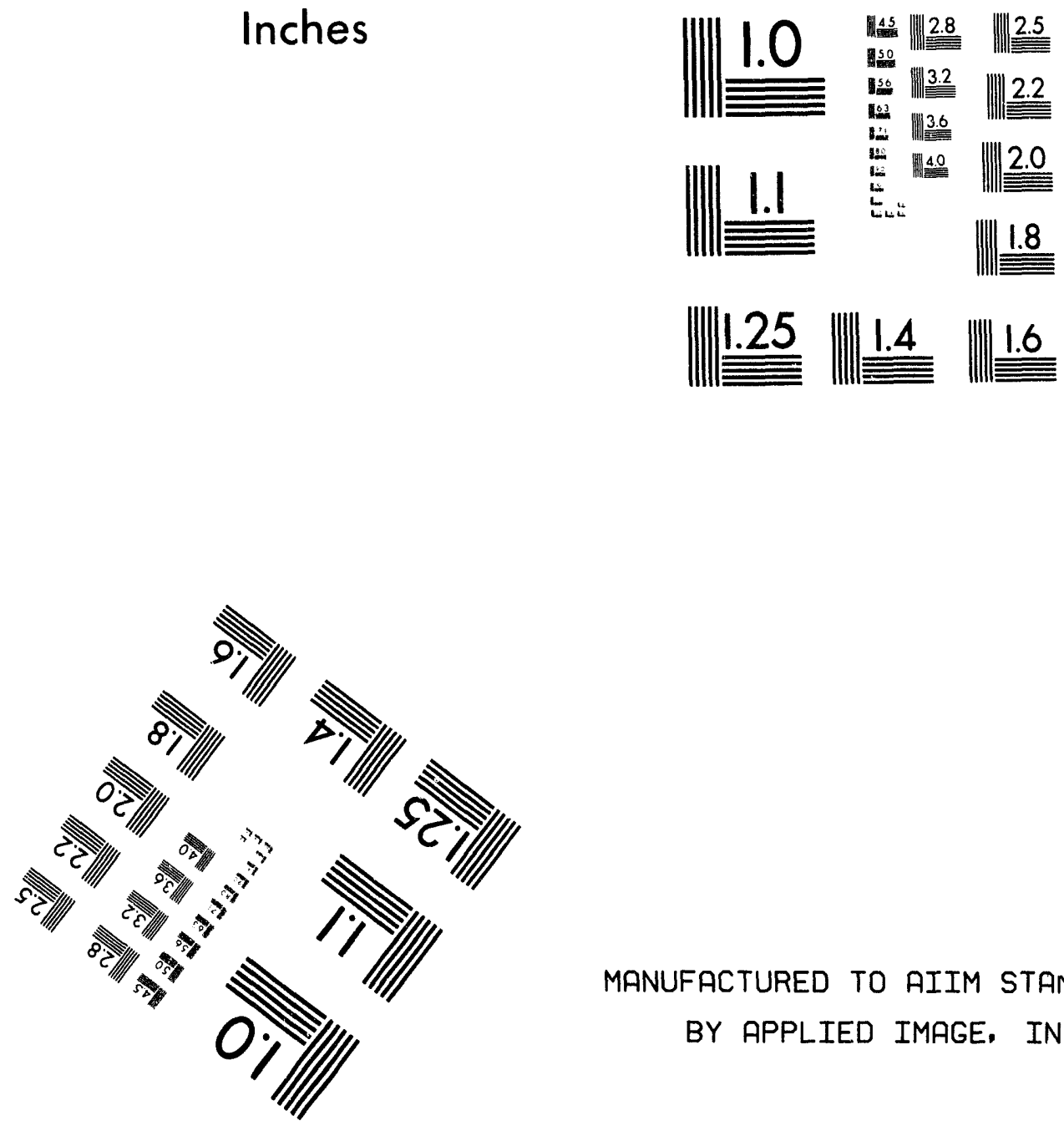

MANUFACTURED TO AIIM STANDARDS BY APPLIED IMAGE, INC.

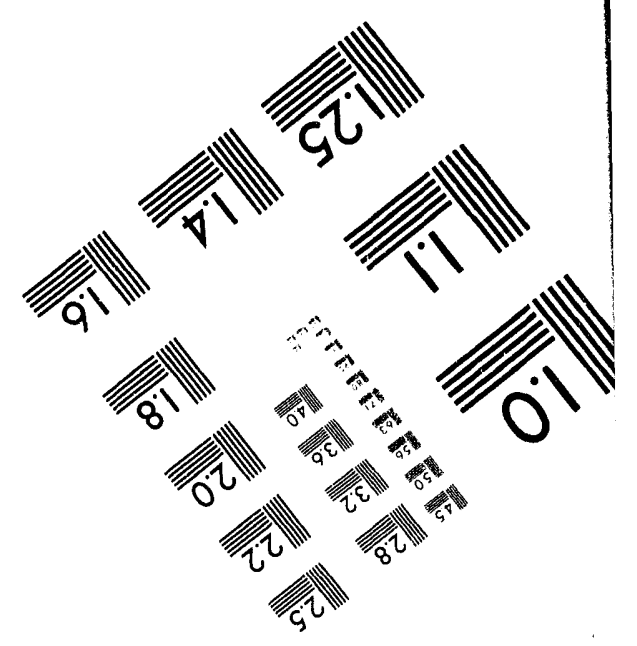



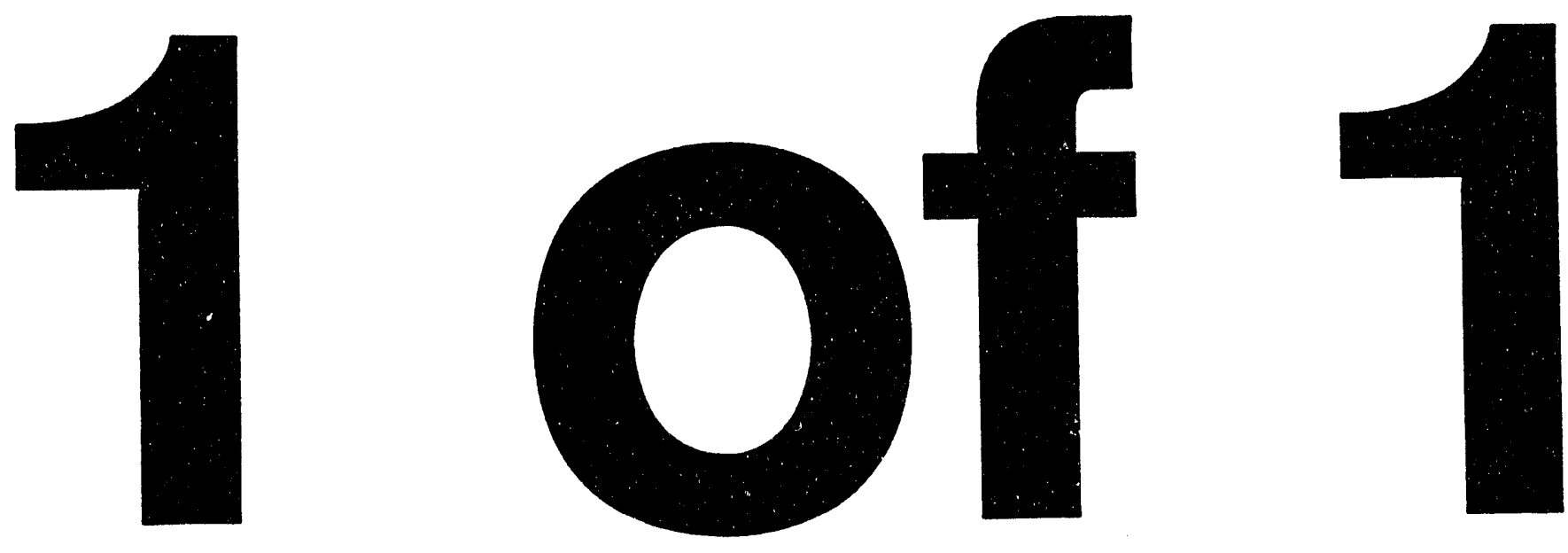


\section{Groundwater Maps of the Hanford Site, December 1993}

G. L. Kasza

M. J. Hartman

W. A. Jordan

J. V. Borghese

Date Published

July 1994

Prepared for the U.S. Department of Energy Office of Environmental Restoration and Waste Management

$\begin{array}{ll}\text { (2) Westinghouse } & \text { P.O. Box } 1970 \\ \text { Hanford Company } & \text { Richland, Washington } 99352\end{array}$

Hanford Operations and Engineering Contractor for the

U.S. Department of Energy under Contract DE-AC06-87RL10930 
CONTENTS

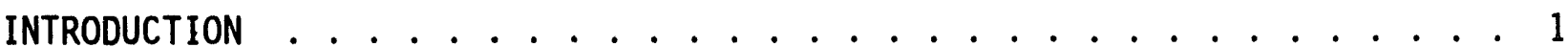

DATA COLLECTION, MANAGEMENT, AND PRESENTATION ........... 2

HANFORD SITE MAPS .................... 2

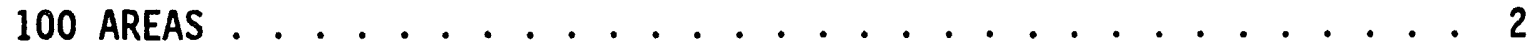

200 AREAS . . . . . . . . . . . . . 3

$300 / 1100$ AREAS .......................... 4

REFERENCES ..................... 5

\section{APPENDICES:}

A Water level Measurement Data . . . . . . . . . . . . . . . A-1

B Water Level Differences, 200 Areas . . . . . . . . . . . . B-1

\section{FIGURES:}

1 Hanford Site Location Map . . . . . . . . . . . . . . . . 6

2100 Areas Index Map . . . . . . . . . . . . . . . . . . . 7

3100 Areas Depth-to-Water Map . . . . . . . . . . . . . . . . 8

4100 Areas Water Table Map . . . . . . . . . . . . . . . . . . . . 9

5200 Areas Index Map ......................... 10

6200 Areas Depth-to-Water Map . . . . . . . . . . . . . . . . . . . . 11

7200 Areas Water Table Elevation . . . . . . . . . . . . . . . . . 12

8200 Areas Change in Water Table Elevation December 1988 to December 1993. . . . . . . . . . . . . . . . . . . 13

$9300 / 1100$ Area Index Map . . . . . . . . . . . . . . . . . . . . . 14

$10300 / 1100$ Area Depth-to-Water Map . . . . . . . . . . . . . . . . . . 15

$11300 / 1100$ Area Water Table Map .. . . . . . . . . . . . . . . . 16 


\section{INTRODUCTION}

The Groundwater Maps of the Hanford Site, December 1993 is an update to of the series of reports that document the configuration of the uppermost unconfined aquifer beneath the Hanford Site (Figure 1). This series presents the latest results of the semiannual water level measurement program and the water table maps generated from these measurements. The reports document the changes in the groundwater level at the Hanford Site during the transition from nuclear material production to environmental restoration and remediation. In addition, these reports provide water level data to support the various site characterization and groundwater monitoring programs currently in progress on the Hanford Site. Groundwater Maps of the Hanford Site is prepared for the U.S. Department of Energy, Office of Environmental Restoration and Waste Management, by the Hanford Site Operations and Engineering Contractor, Westinghouse Hanford Company (WHC). This document fulfills reporting requirements specified in WHC (1993), Section 8.0 "Water Quality" and also described in the environmental monitoring plan for the Hanford Site (DOE-RL 1991).

The three major operations areas (the 100, 200 and 300/1100 Areas) where wastes were discharged to the soil are covered in this update. The water level measurements from the wells in these areas are portrayed on a set of maps to illustrate the hydrologic conditions and are also tabulated in an appendix. A summary discussion of the data is included with the well index map, the depth to water map, and the contoured map of the water table surface for each of the three areas. A map depicting the change in water table elevation since 1988 is included for the 200 Area. Appendix A 1 ists the well identification number, depth to water, casing elevation and the water level elevation for each well measured during December 1993. Appendix B 1ists the 1988 and 1993 water level measurements and the difference between those measurements for the 200 Area. For clarity, the locating prefixes have been omitted from all well numbers shown on the maps. Wells in the 100 Areas have the prefix 199; those in the 200 Areas have the prefix 299; wells in the 300 Area have a 399 prefix; those in the 1100 Area have a 1199 prefix and the wells outside these areas have the prefix 699. An explanation of the Hanford well numbering system and a listing of the survey coordinates for each well drilled prior to 1993 is listed in Chamness and Merz (1993).

The National Geodetic Vertical Datum of 1929 is used as the vertical datum and Hanford Plant coordinates used fur horizontal location of the wells. Both datums are in feet.

Water level data collected in June 1993 during the previous Hanford Site semiannual water table measurement field effort are reported in Kasza et al. (1994). 


\section{DATA COLLECTION, MANAGEMENT, AND PRESENTATION}

During December 1993, the depth to the groundwater was measured in 738 Hanford Site wells by personnel from Subsurface Investigative Support Function, under the direction of the Geohydrologic Engineering Function project ieaders. The procedure for measuring the depth to water and recording the data is contained in environmental investigations instruction 10.2 , Environmental Investigation and Site Characterization Manual (WHC 1988). Field data were collected using the Groundwater Monitoring System, which employs a barcode interface to enter readings that have been taken manually with a steel tape from the reference point at the top of the well casing. The barcode hand-held computer downloads the field data to a supervisory workstation that stores all groundwater level measurements. These data are transferred to the Hanford Environmental Information System (HEIS) data base and were later downloaded to a Paradox (a trademark of Borland International) application program for use by the Geohydrologic Engineering function hydrologists.

The Paradox application was used to classify, locate, and format data points used in creating contours of the water table across the Hanford Site. Data were averaged for wells with multiple measurements in December 1993. The Paradox application was also used to generate the data tables presented in Appendix A.

Contours generated from groundwater data are displayed on the water table elevation maps and were created within Earth Vision (a trademark of Dynamic Graphics, Inc.). Earth Vision is software that calculates a data grid and generates contour lines when given a set of data point locations and values. A 2-D minimum tension gridding algorithm was used to interpolate grid values. Generated contour lines were then imported into an AutoCad (a trademark of Autodesk, Inc.) environment to be overlaid onto existing base maps.

iHdrologists familiar with regional and local groundwater properties reviewed the maps to evaluate data interpolations made by the Earth Visior software. A representative set of wells was illustrated in areas where there are heavy concentrations of wells (e.g., 100-N and 100-H Areas). Hydrographs were plotted for wells with anomalous head values. If the measurement was an outlier, the data point was not included in constructing the maps and its questionable status was noted in Appendix A. Map modifications and corrections were made in AutoCad and the final maps were generated.

\section{HANFORD SITE MAPS}

\section{AREAS}

For the purposes of this report, the 100 Areas comprise the various 100 Area reactor facilities and the surrounding land south of the Columbia River and north of Gable Mountain and Gable Butte. Reactor operations have 
ceased in all of the facilities, and environmental restoration activities are in progress. Maps for this area include:

- Figure 2, 100 Areas Index Map

- Figure 3, 100 Areas Depth-to-Water Map

- Figure 4, 100 Areas Water Table Map.

The maps are based on December 1993 field measurements from those wells that are located north of Hanford Site coordinate $N 56,000$ and completed in the unconsolidated sediments. Wells used are completed within $100 \mathrm{ft}$ of the average water table since vertical gradients are not significant on the scale of the map.

Data were averaged for wells with multiple measurements in December 1994. A representative set of wells from the reactor areas was chosen where there are heavy concentrations of wells (e.g., $100-\mathrm{N}$ and 100-H Areas). Hydrographs were plotted for wells with anomalous head values. If the measurement was an outlier, the data point was not included in constructing the maps.

River stage recorders are located in the $100-\mathrm{B} / \mathrm{C}, 100-\mathrm{N}, 100-\mathrm{H}$, and 100-F Areas. River stage is measured hourly with pressure transducers. The averages of the December values are plotted on Figure 4 and were used in contouring the water table map.

Throughout most of the map area, groundwater flows from the unconfined aquifer into the Columbia River. West of the 100-B/C Area, water is believed to flow from the river into the aquifer.

The high water levels north of Gable Mountain at wells 699-66-38, 69966-39, and 699-69-38 are consistent with past measurements. Driller's logs indicate the presence of fine-grained sediments in this area. The high water levels may be remnants of artificial recharge from pre-Hanford irrigation, or may represent perched groundwater (Kasza et al. 1990). The steep groundwater gradient in the southeastern portion of the map area is believed to result from the influence of the fine-grained sediments.

Water level data used to construct the maps are listed in Appendix $A$. The $N 65,000$ match line is included on the maps of the 100 and 200 Areas for convenience.

\section{AREAS}

These maps encompass the 200 East and 200 West Areas and the surrounding vicinity on the Hanford Site that was once referred to as the Separations Area. The semiannual measurement of water levels in the 200 Areas was performed on the more than 180 selected wells that comprise the Operational Groundwater Monitoring Network (Serkowski and Jordan, 1989). The Operational Groundwater Monitoring Network provides water level measurements for the determination of the water table configuration and for the water quality sampling of the groundwater beneath and surrounding the chemical processing and waste management facilities in the 200 Areas. Water level data from RCRA program, CERCLA program, and outlying wells are included on the maps to 
provide supplemental information for a more complete interpretation. Wells selected for use were reviewed to assure that they were completed in the unconsolidated sediments and screened or perforated in the upper part of the unconfined aquifer.

The 200 Areas set of maps consists of:

- Figure 5, 200 Areas Index Map

- Figure 6, 200 Areas Depth-to-Water Map

- Figure 7, 200 Areas Water Table Elevation

- Figure 8, 200 Areas Change in Water Table Elevation December 1988 to December 1993.

The "areas where the basalt surface is above the water table" reflects the top of the basalt according to Connelly (1992a, 1992b). Representative wells were selected to portray the water level data on the maps where the concentration of monitoring wells would produce a crowded illustration.

Figure 8 depicts the change in the water table beneath the 200 Areas that occurred between December 1988 and December 1993. December 1988 was selected because at that time, Hanford had not ceased its defense production role and the water table was at the highest elevation in recent years. This map shows the general lowering of the water table in the 200 Areas in response to the decrease in disposal of process water. The location of the greatest decrease in the 200 West Area is the site of the abandoned U-Pond while the greatest decrease in the 200 East Area corresponds to effluent disposa? locations for the PUREX PIant. The water table in the area east of 200 East Area has not declined as rapidly due to the continued discharges at B-Pond. Only data from monitoring wells measured during the period late November to Early January 1988 and December 1993 were used to construct the contours. The water level data used for this map are found in Appendix B.

\section{$300 / 1100$ AREAS}

This section contains a discussion on the December 1993 water table measurements for the 300 Area and the adjacent 1100 Area Horn Rapids Landfill. Wells used for obtaining water level measurements were screened or perforated in the upper part of the unconfined aquifer except in a few cases where the top of the screen was slightly below. The data for this area are presented in Figures 9 and 10, the index and depth-to-water maps, respectively. The $300 / 1100$ Area water table map is shown in Figure 11.

The unconfined aquifer within this area is contained in the Ringold Formation and the Hanford formation (Swanson et al. 1992). The top of the unconfined aquifer is close to the Hanford/Ringold contact. West of an imaginary north-south line near the west boundary of the 300 Area the unconfined aquifer is generally within the Ringold Formation. East of the line it is generally within the Hanford formation.

The major influences on water table elevations in the map area are river fluctuations, irrigation, and river water recharged into the city of Richland well field near the 1100 Area. Water table elevation may also be dependent on recharge from the Yakima River to the west. 
WHC-EP-0394-8

\section{REFERENCES}

Chamness, M. A. and J. K. Merz, 1993, Hanford Wells, PNL-8800, Pacific Northwest Laboratory, Richland, Washington.

Connelly, M. P., B. H. Ford, and J. V. Borghese, 1992a, Hydrogeologic Model for the 200 West Groundwater Aggregate Area, WHC-SD-EN-TI-014, Westinghouse Hanford Company, Richland, Washington.

Connelly, M. P., B. H. Ford, J. W. Lindberg, S. J. Trent, C. D. Delaney, and J. V. Borghese, 1992b, Hydrogeologic Model for the 200 East Groundwater Aggregate Area, WHC-SD-EN-TI-019, Westinghouse Hanford Company, Richland, Washington

DOE-RL, 1991, Environmental Monitoring P7an, DOE/RL 91-50, U.S. Department of Energy, Richland Operations Office, Richland, Washington.

Kasza, G. L., S. F. Harris, and M. J. Hartman, 1990, Groundwater Maps of the Hanford Site--June 1990, WHC-EP-0394-1, Westinghouse Hanford Company, Richland, Washington.

Kasza, G. L., M. J. Hartman, W. A. Jordan, and D. C. Weekes, 1994, Ground Water Maps of the Hanford Site--June 1993, WHC-EP-0394-7, Westinghouse Hanford Companj, Richland, Washington.

Serkowski, J. A. and W. A. Jordan, 1989, Operational Groundwater Monitoring at the Hanford Site--1988, WHC-EP-0260, Westinghouse Hanford Company, Richland, Washington.

Swanson, L. C., G. G. Kelty, K. A. Lindsey, K. R. Simpson, R. K. Price, and S. $D$. Consort, 1992, Phase I Hydrogeologic Summary of the 300-FF-5 Operable Unit, 300 Area, WHC-SD-EN-TI-052, Westinghouse Hanford Company, Richland, Washington.

WHC, 1988, Environmental Investigations and Site Characterization Manual, WHCCM-7-7, Westinghouse Hanford Company, Richland, Washington.

WHC, 1993, Environmental Compliance, WHC-CM-7-5, Westinghouse Hanford Company, Richland, Washington. 


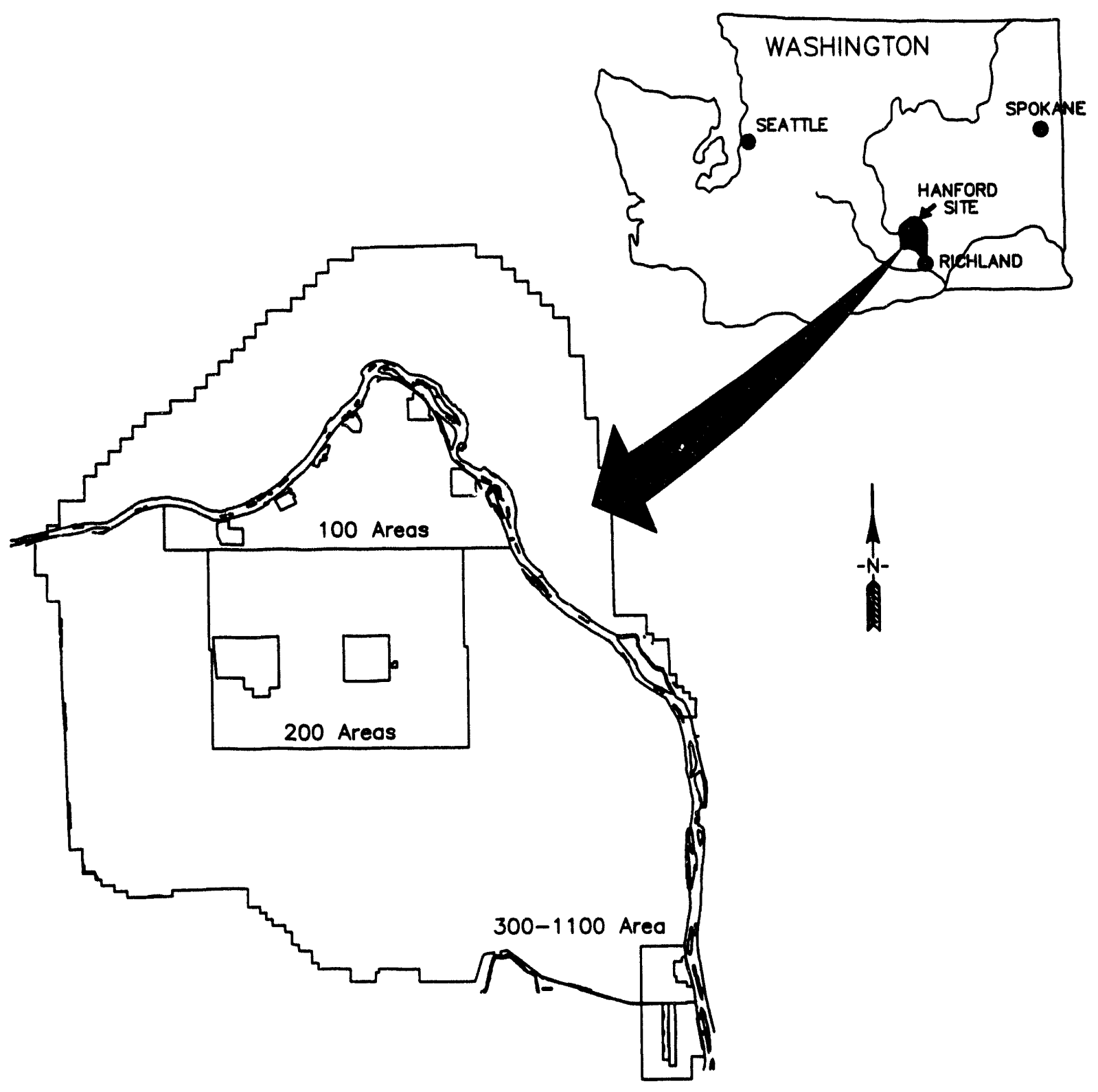

Figure 1. Hanford Site Location Map. 


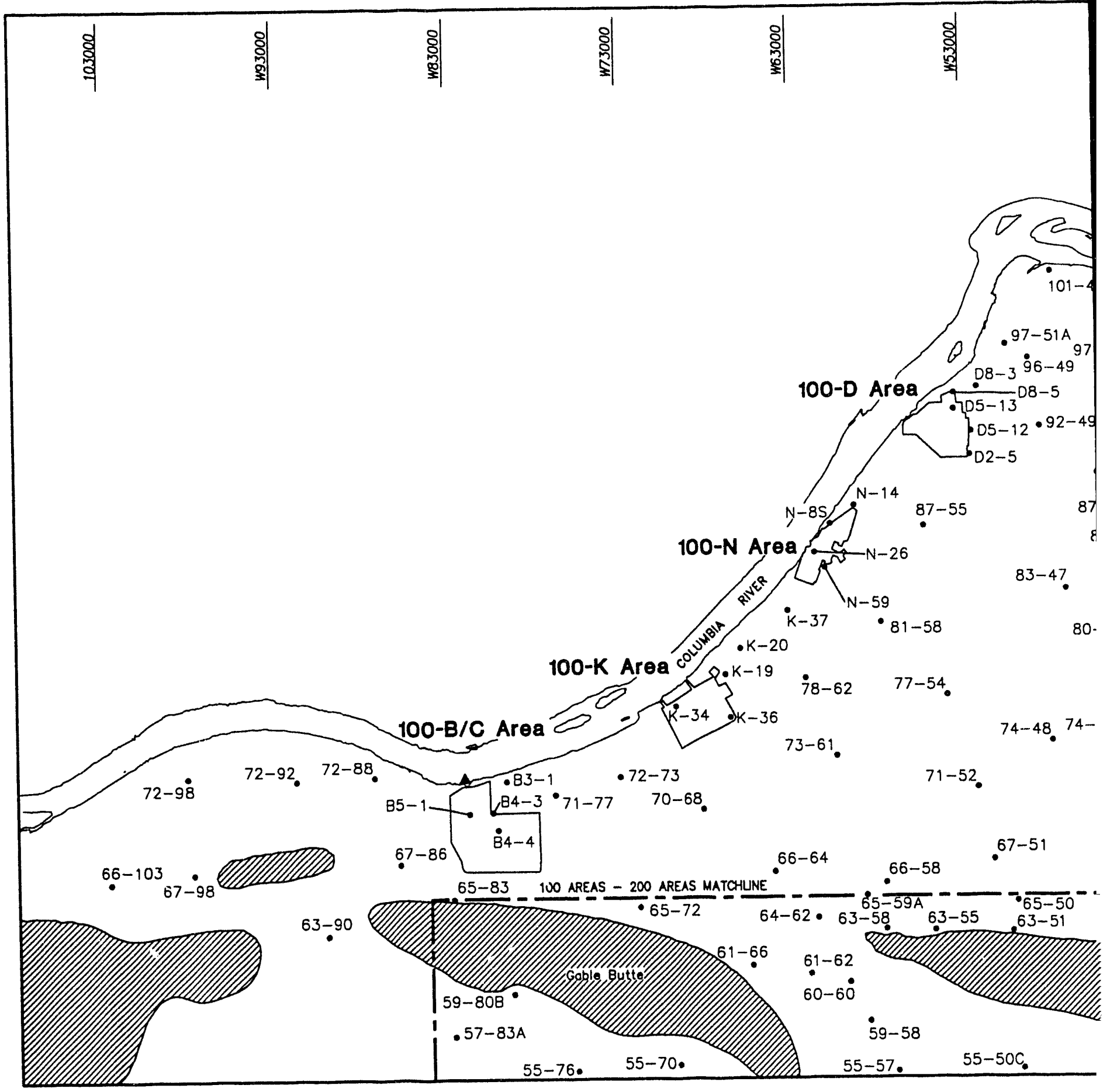




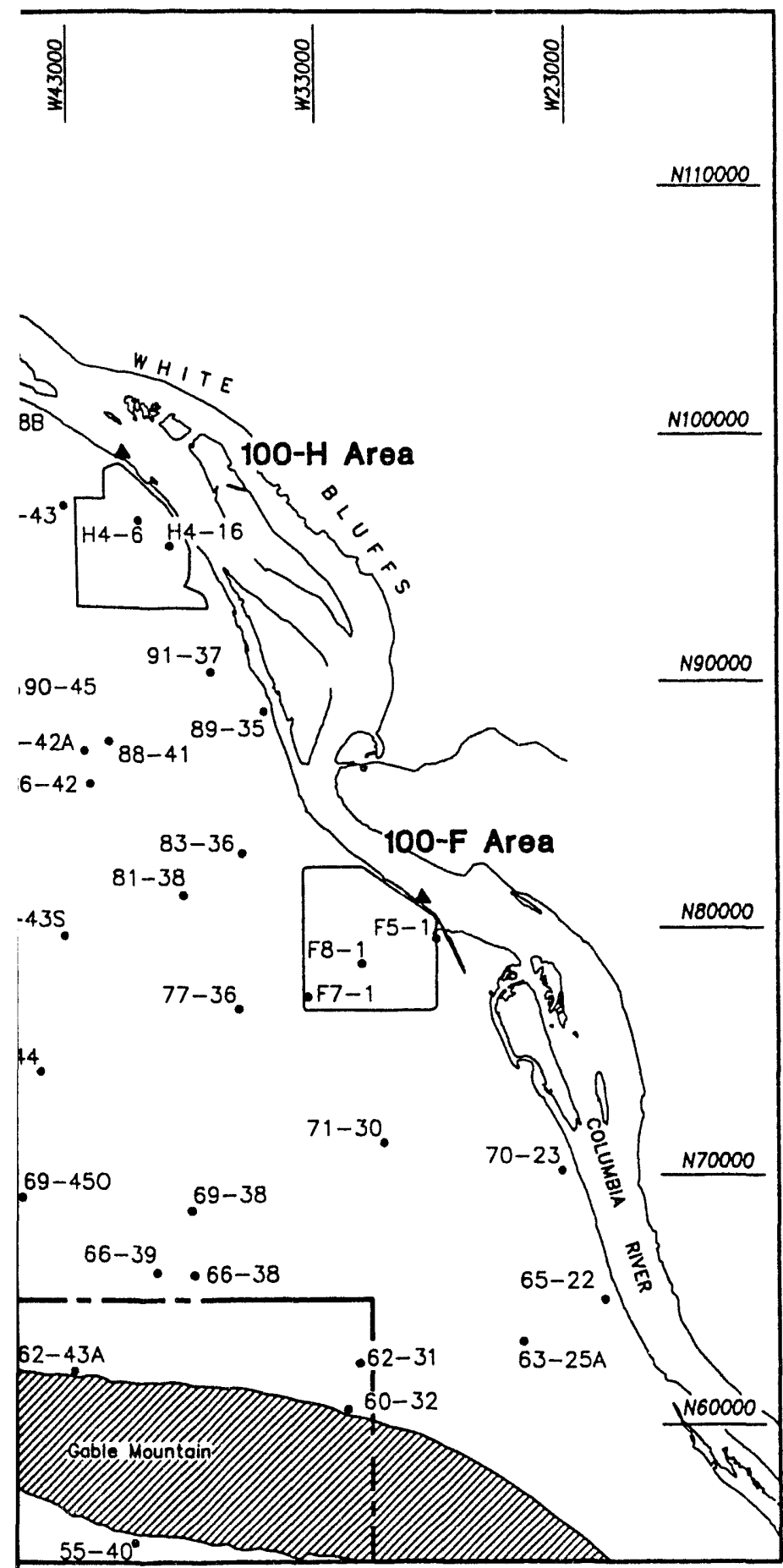

WAJ/1IDXDC93
Figure 2

\section{Areas Index Map December 1993}

\begin{abstract}
Identification of Groundwater monitoring Wells Used to Prepore this map. Well numbers beginning with a letter are prefixed with a with a 199- and beginning with 0 number are prefixed with 699-.
\end{abstract}

- River stage recorder

Areas where the basalt surface is generally above the water table

The 100 Areas Index Map has been prepared by the Geohydrologic Engineering Function, Westinghouse Hanford Company.

Note: To convert to metric, multiply elevation ( $\mathrm{ft}$ ) by 0.3048 to obtain elevation $(\mathrm{m})$.

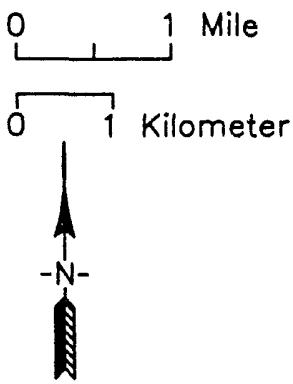




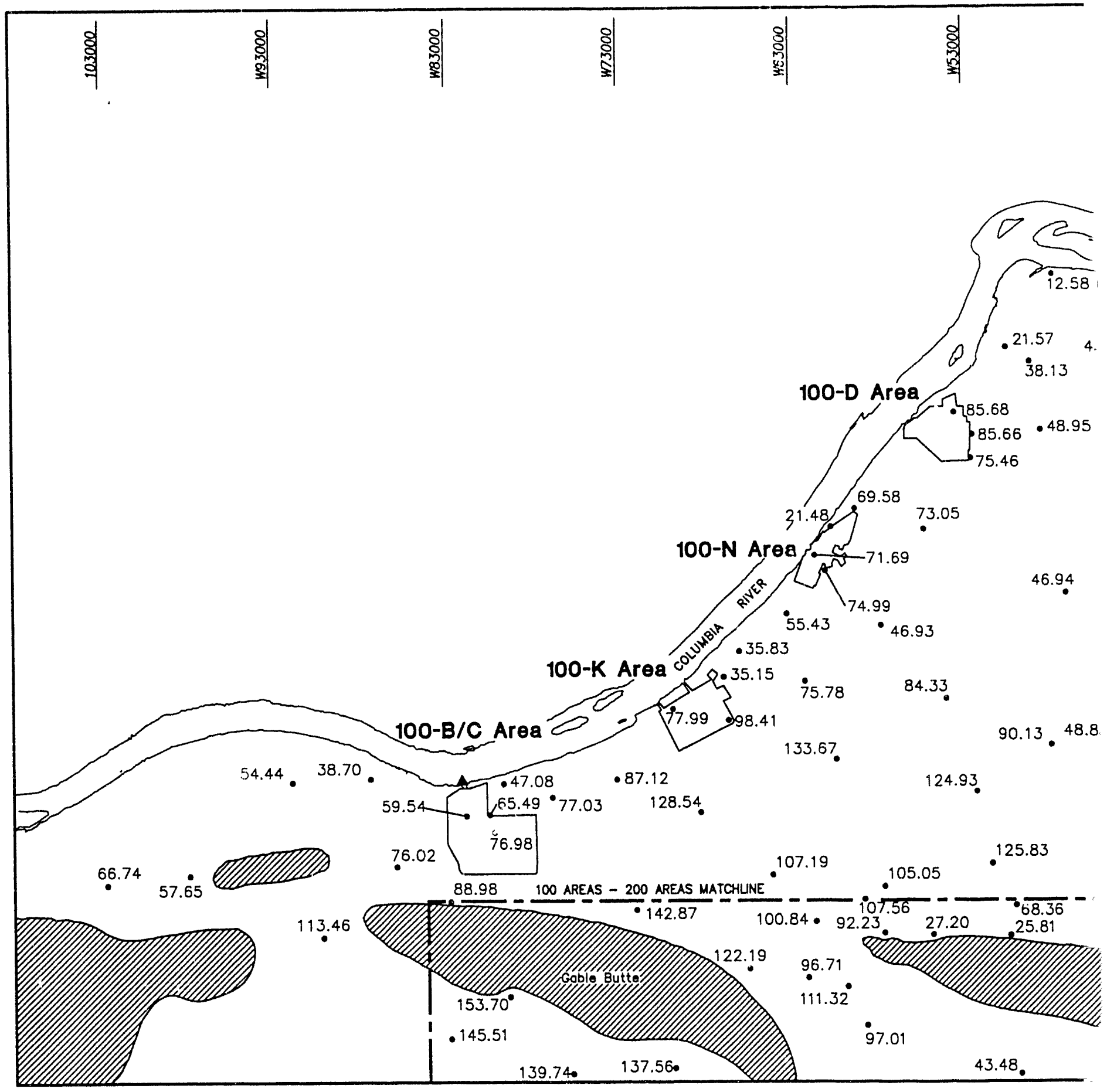


WHC-EP-0394-8

Figure 3

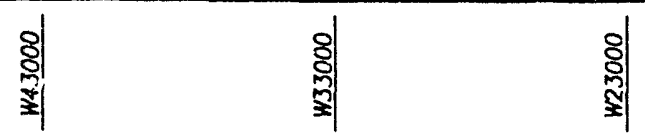

100 Areas Depth-to-Water Map December 1993

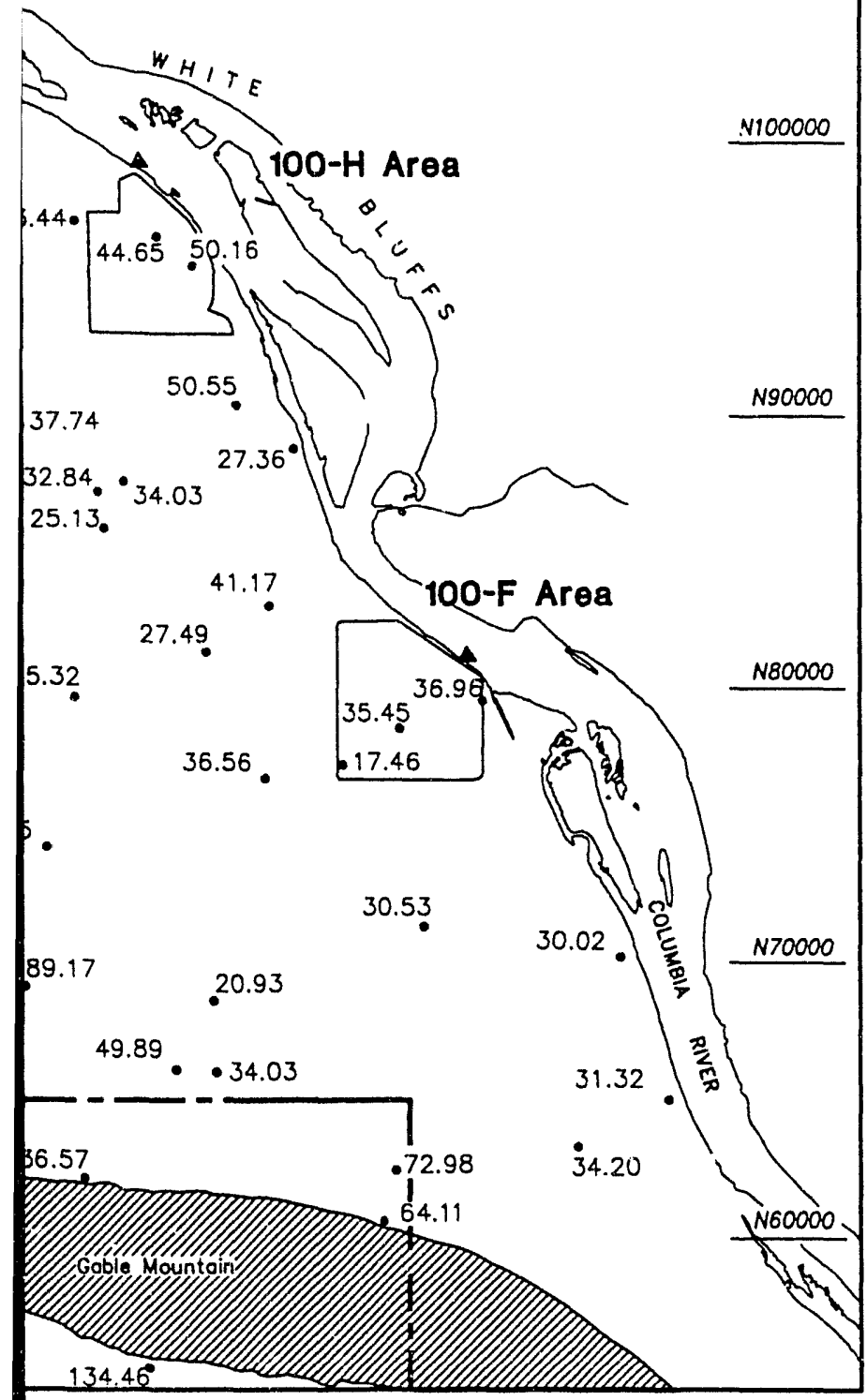

Depth to water, as measured from the well reference mark (generally top of cosing) to groundwater surface.

Fiver stage recorder

Areas where the basalt surface is generally above the woter table.

The 100 Areos depth-to-water map has been prepared by the Geohydrologic Support Function, Westinghouse Hanford Company.

Note: To convert to metric, multiply elevation (ft) by 0.3048 to obtain elevation $(\mathrm{m})$.

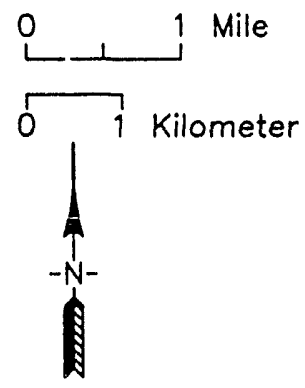

WAJ/1OTWDC93 


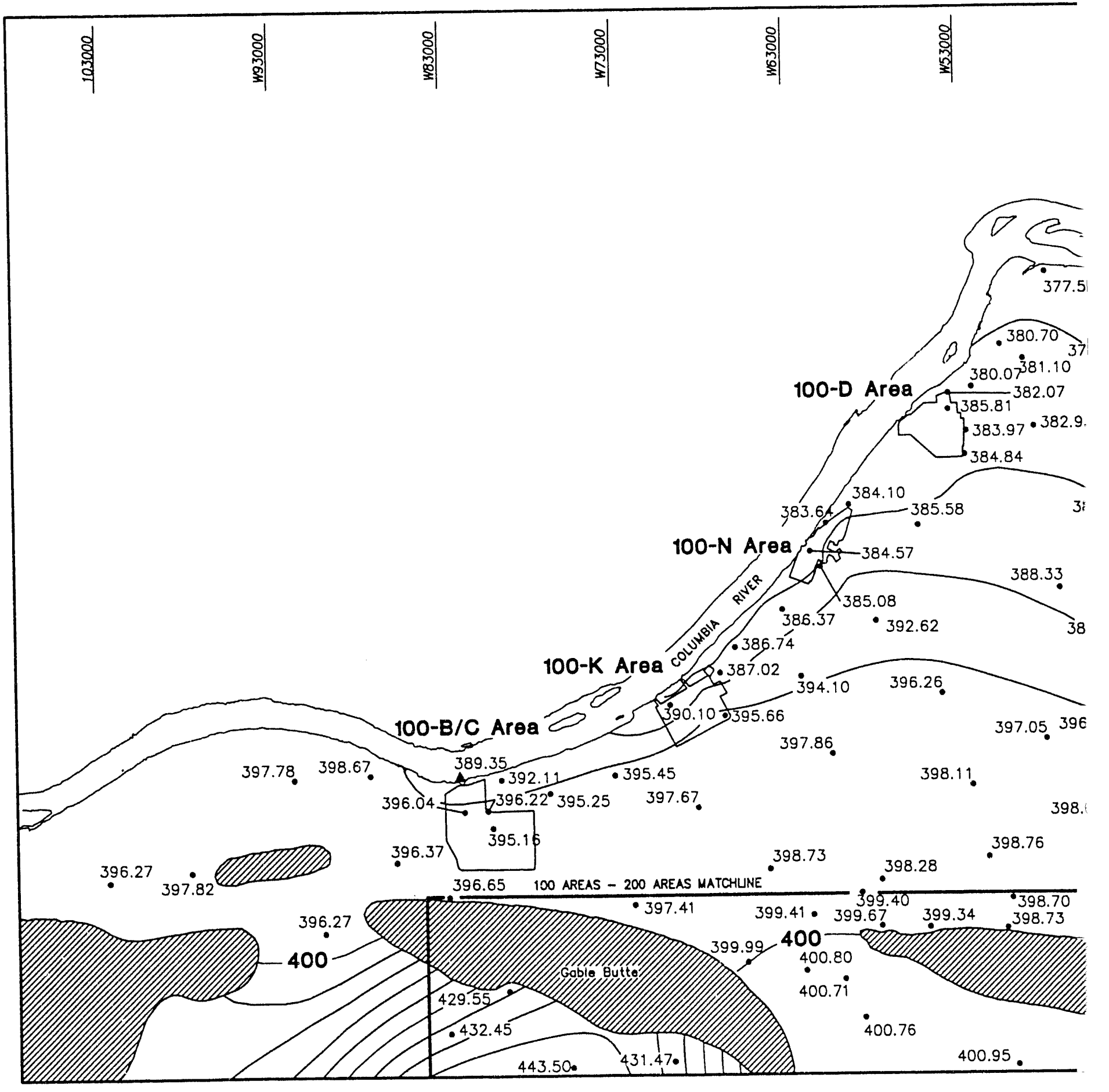


WHC-EP-0394-8

Figure 4

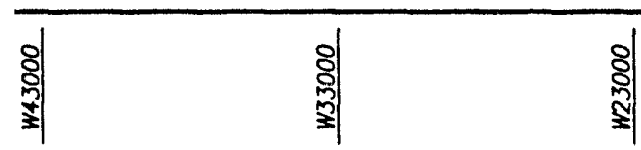

\section{Areas Water Table Map December 1993}
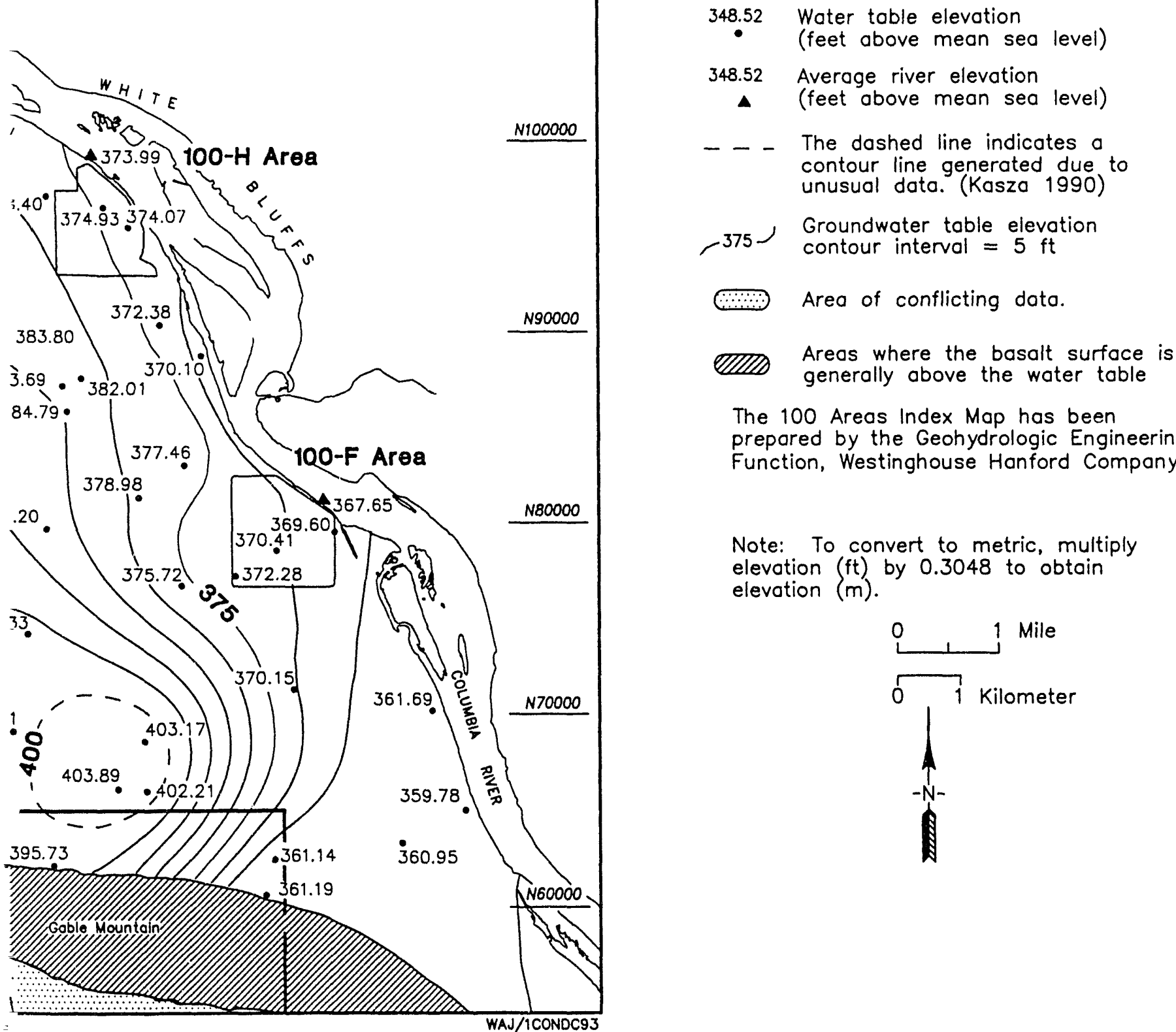

The 100 Areas Index Map has been prepared by the Geohydrologic Engineering Function, Westinghouse Hanford Company.

Note: To convert to metric, multiply elevation (ft) by 0.3048 to obtain elevation (m).

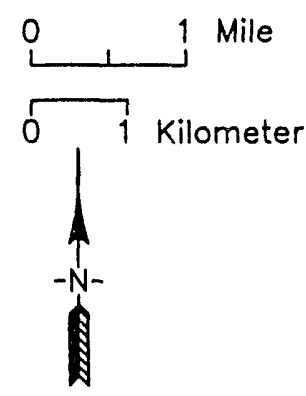




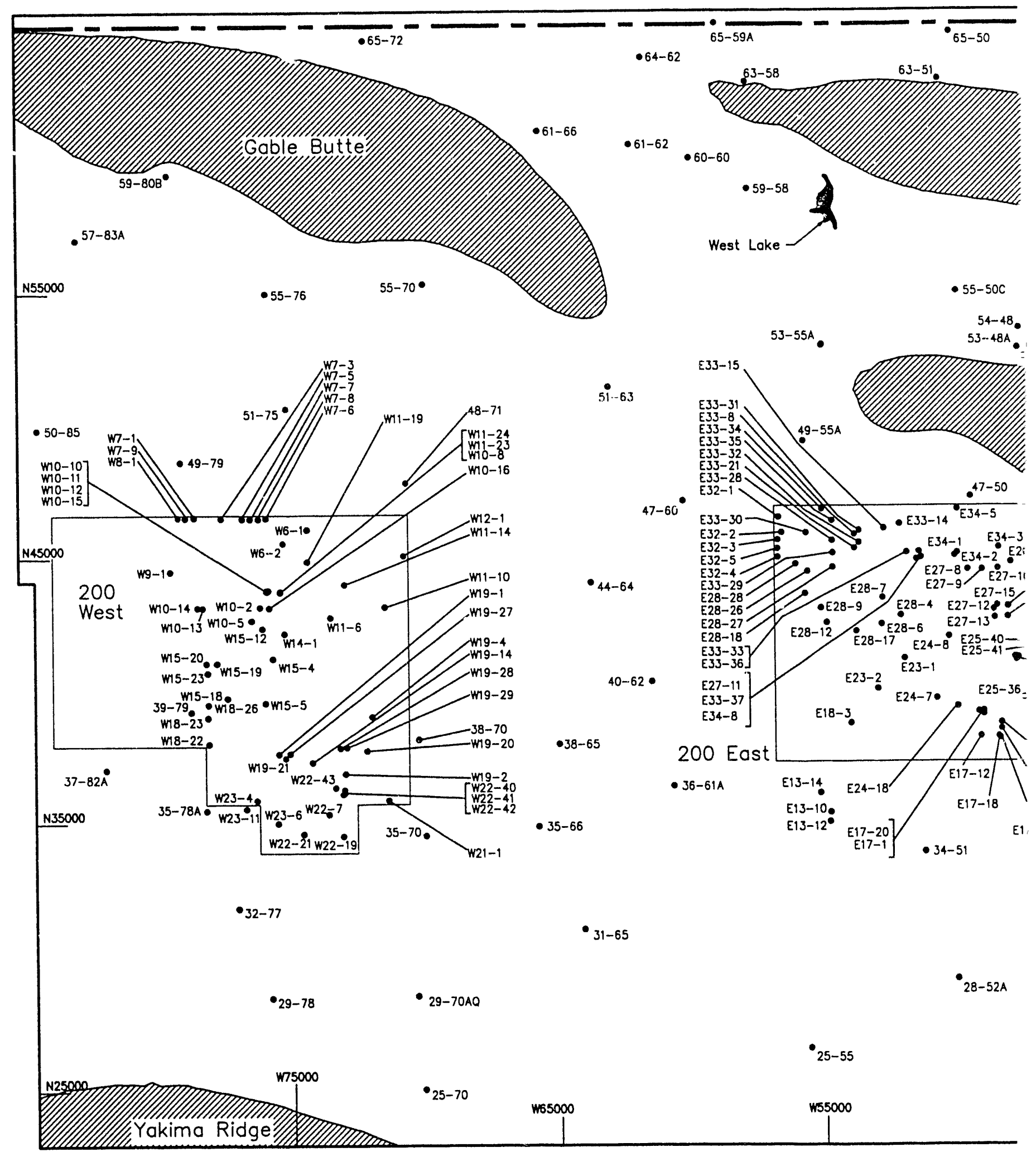



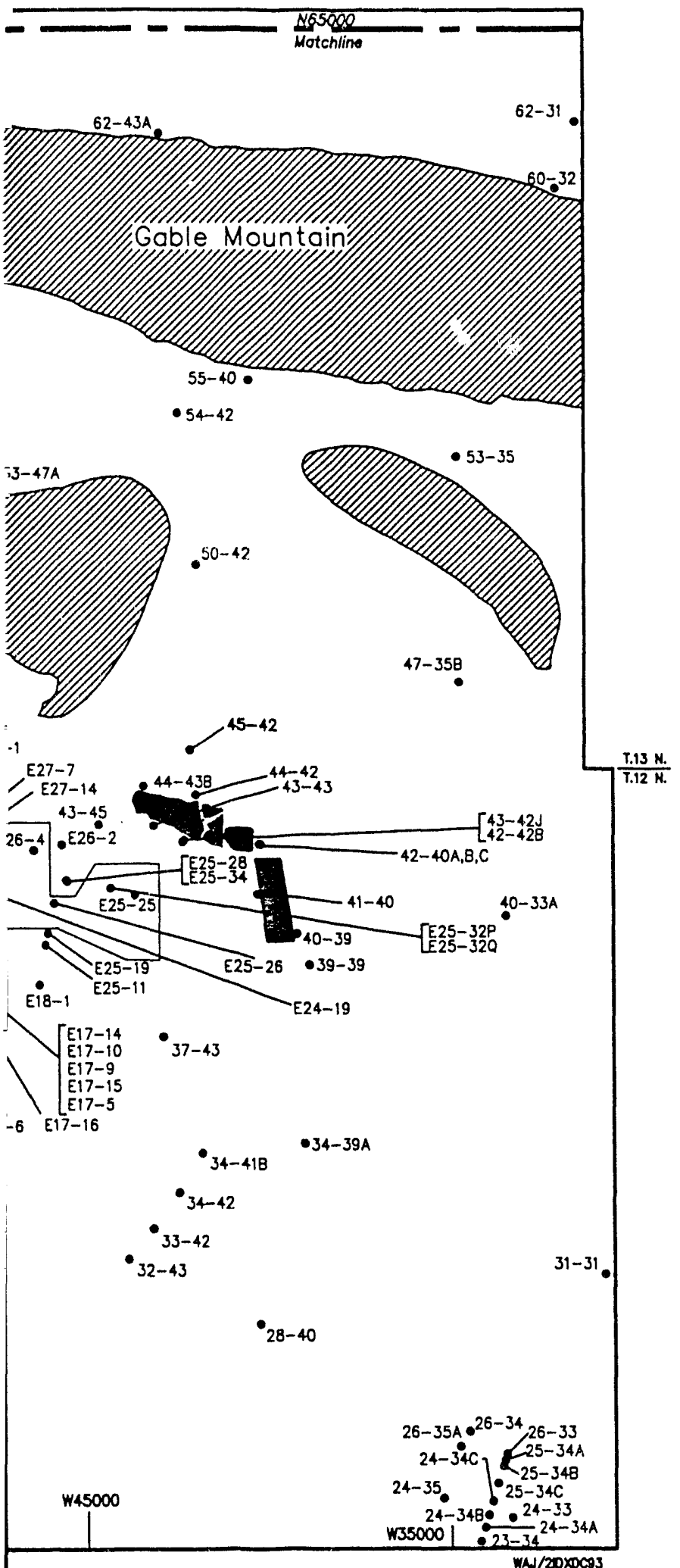

Figure 5

\section{Areas Index Map December 1993}
W22-26 Identification of monitoring well used to prepare map. Well Numbers starting with $E$ or $W$ are prefixed by $299-$ and all others are prefixed by $699-$.

QDPDD

\section{Ponds}

Areos where the basalt surface is generally above the water table

The 200 Areas index map has been prepored by the Geohydrologic Support Function, Westinghouse Hanford Compony.

Note: To convert to metric, multiply elevotion ( $\mathrm{ft}$ ) by 0.3048 to obtoin elevation $(\mathrm{m})$.

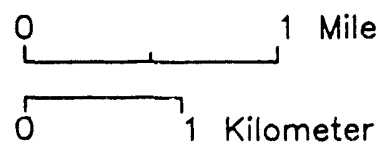




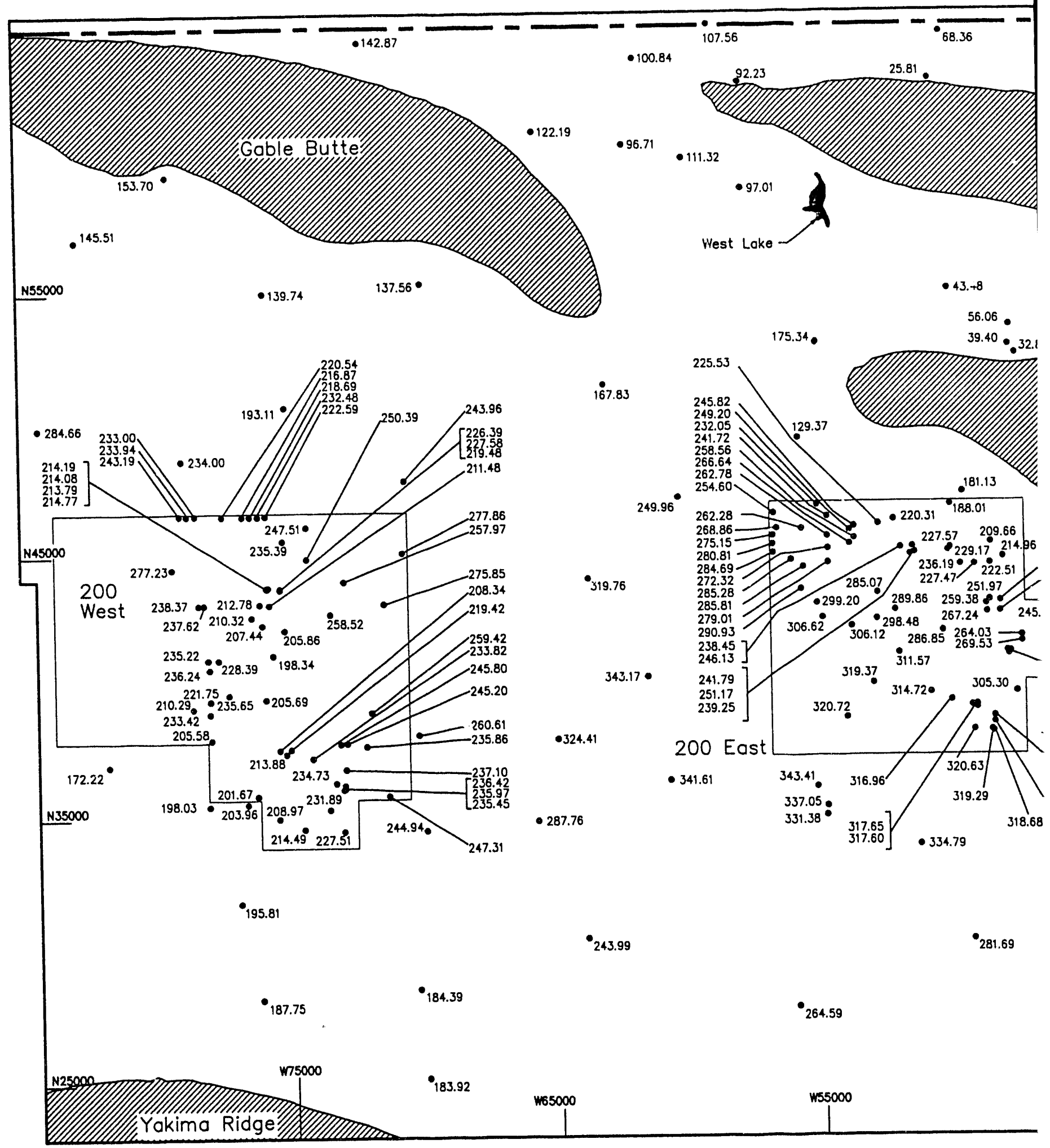




\title{
Figure 6
}

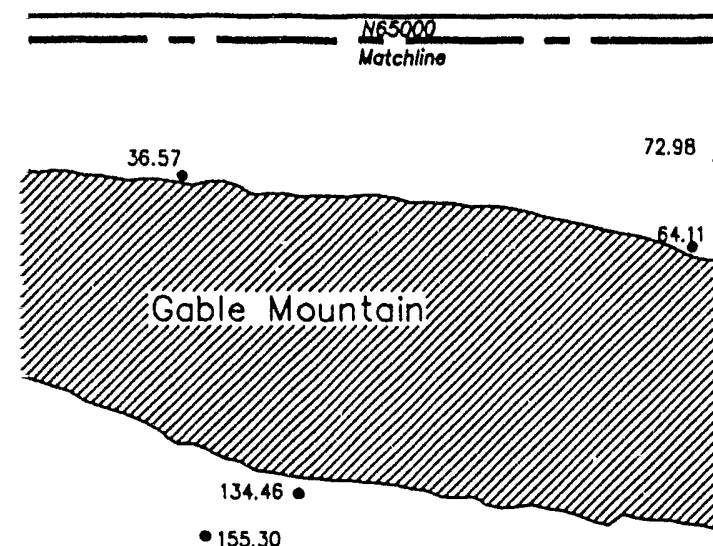

\section{Areas Depth-to-Water Map December 1993}

\author{
206.34 Deoth to water in feet, as \\ - measured from well reference \\ mark (generally top of cosing) \\ to groundwoter surface.
}

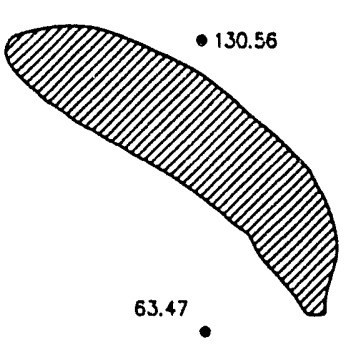

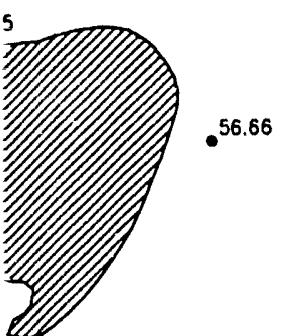

$\bullet 56.66$

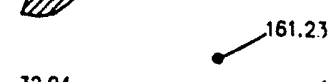

32.94

256.74

194.78

4. 233.00

$164.77 \quad-158.55$
-164.40

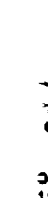

?

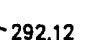

$-\left[\begin{array}{l}260.30 \\ 260.83\end{array}\right.$

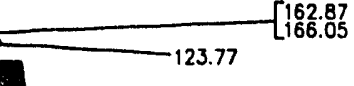

162.87
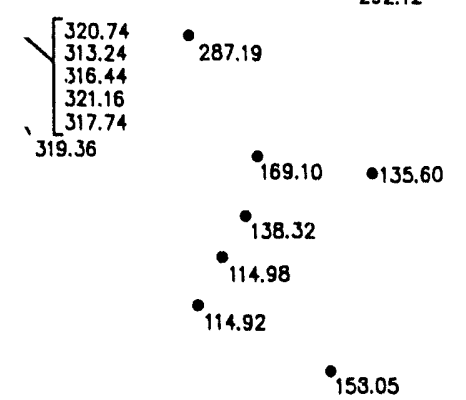

-

287.19

3.05

The 200 Areas depth-to-woter map has been prepared by the Geohydrologic Support Function, Westinghouse Honford Company.

Note: To convert to metric, multiply elevation (ft) by 0.3048 to obtain elevation $(\mathrm{m})$.
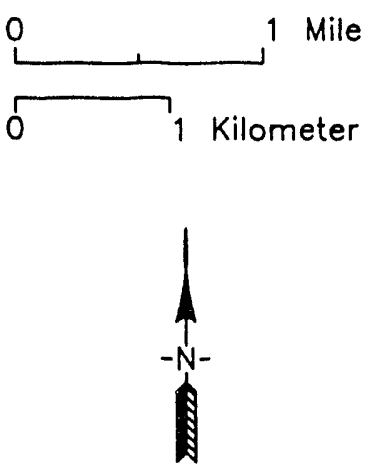


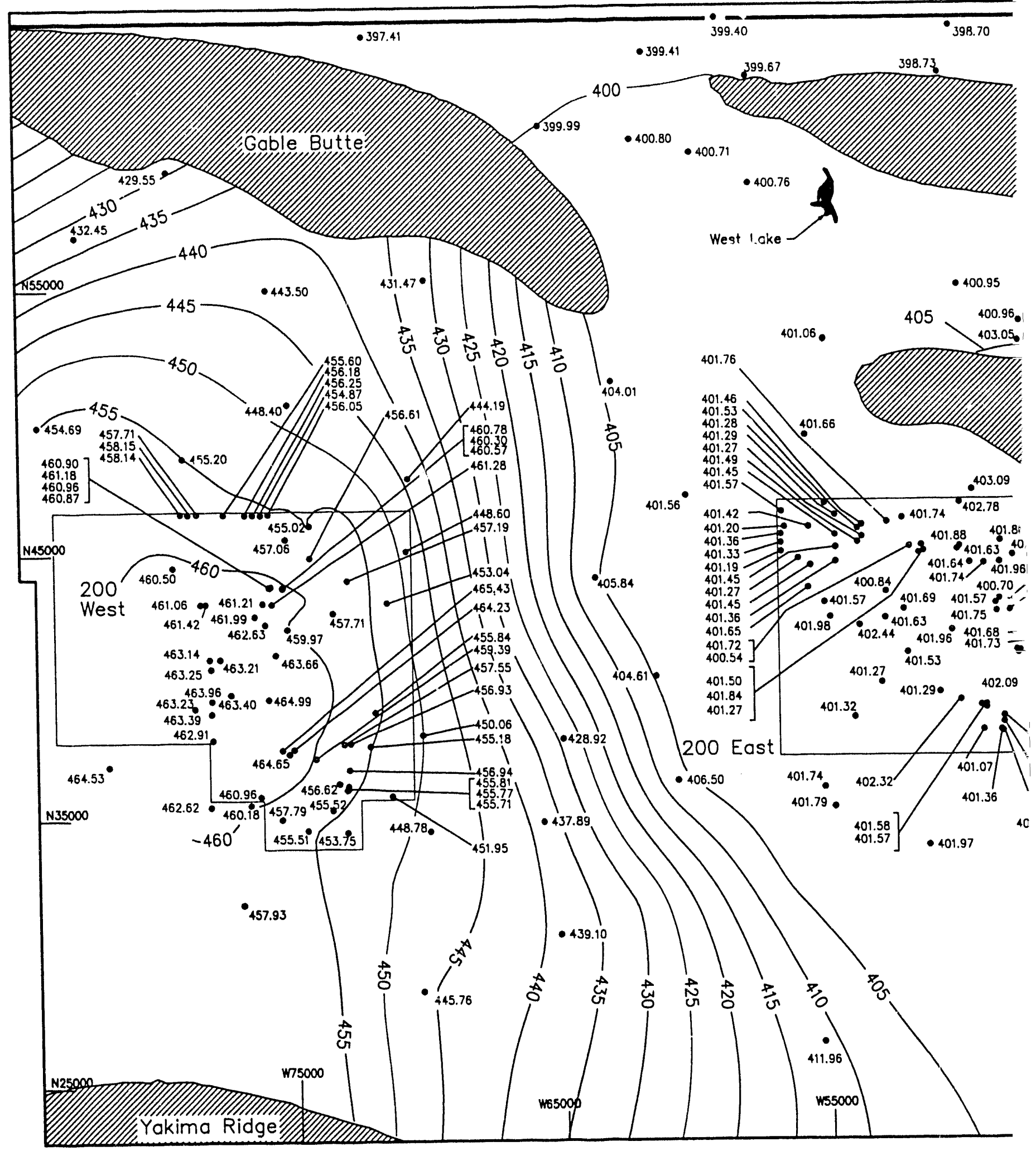




\title{
Figure 7
}

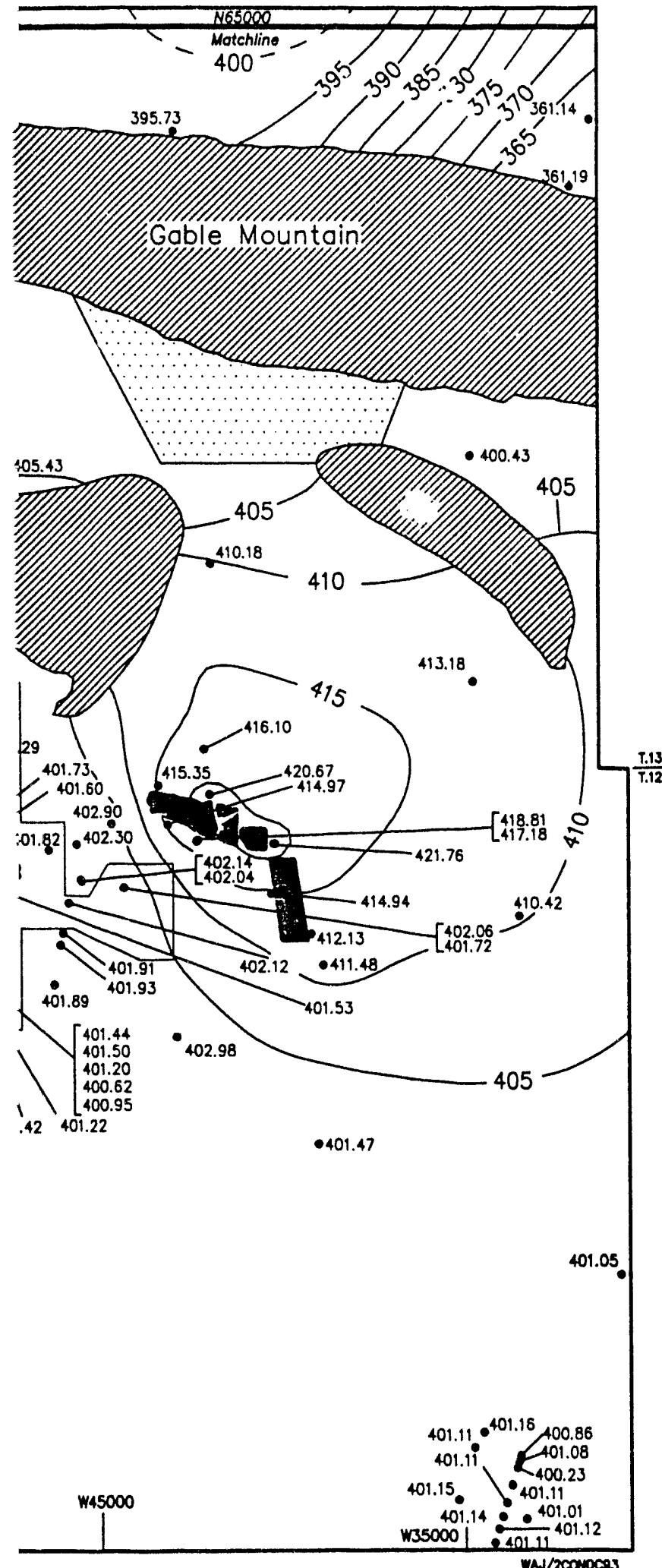

\section{Areas Water Table Elevation December 1993}

\author{
206.34 Water table elevation (feet \\ - above mean sea (evel) \\ - - - The dashed line indicates a \\ contour line generated due to \\ unusual dota. (Kaszo 1990) \\ $400-$ Groundwater table elevation \\ contour interval $=5 \mathrm{ft}$

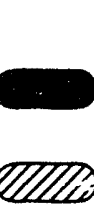 \\ Ponds \\ Areas where the bosalt surfoce is \\ generally above the water table \\ $\therefore$ Areas of conflicting dota.
}

The 200 Areas woter level elevation map has been prepared by the Geohydrologic Support Function, Westinghouse Hanford Company.

Note: To convert to metric, multiply elevation (ft) by 0.3048 to obtain elevation $(m)$.
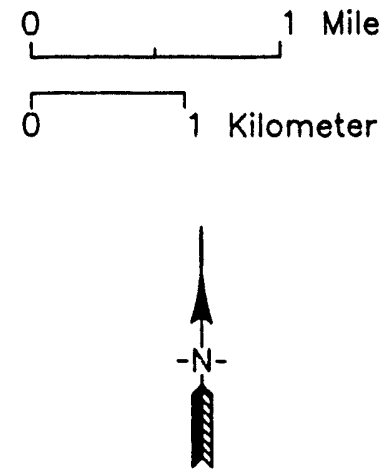


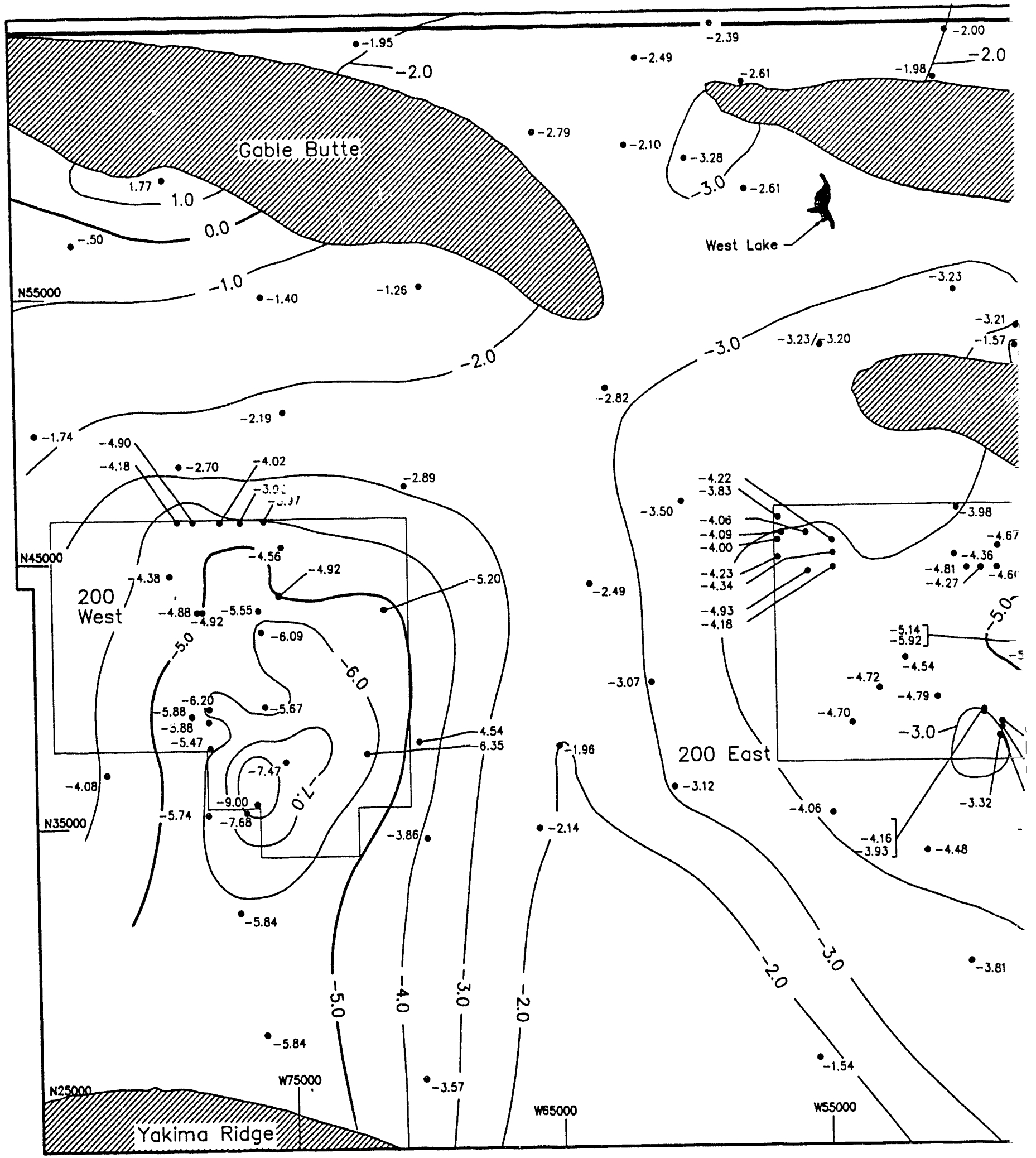




\title{
Figure 8
}

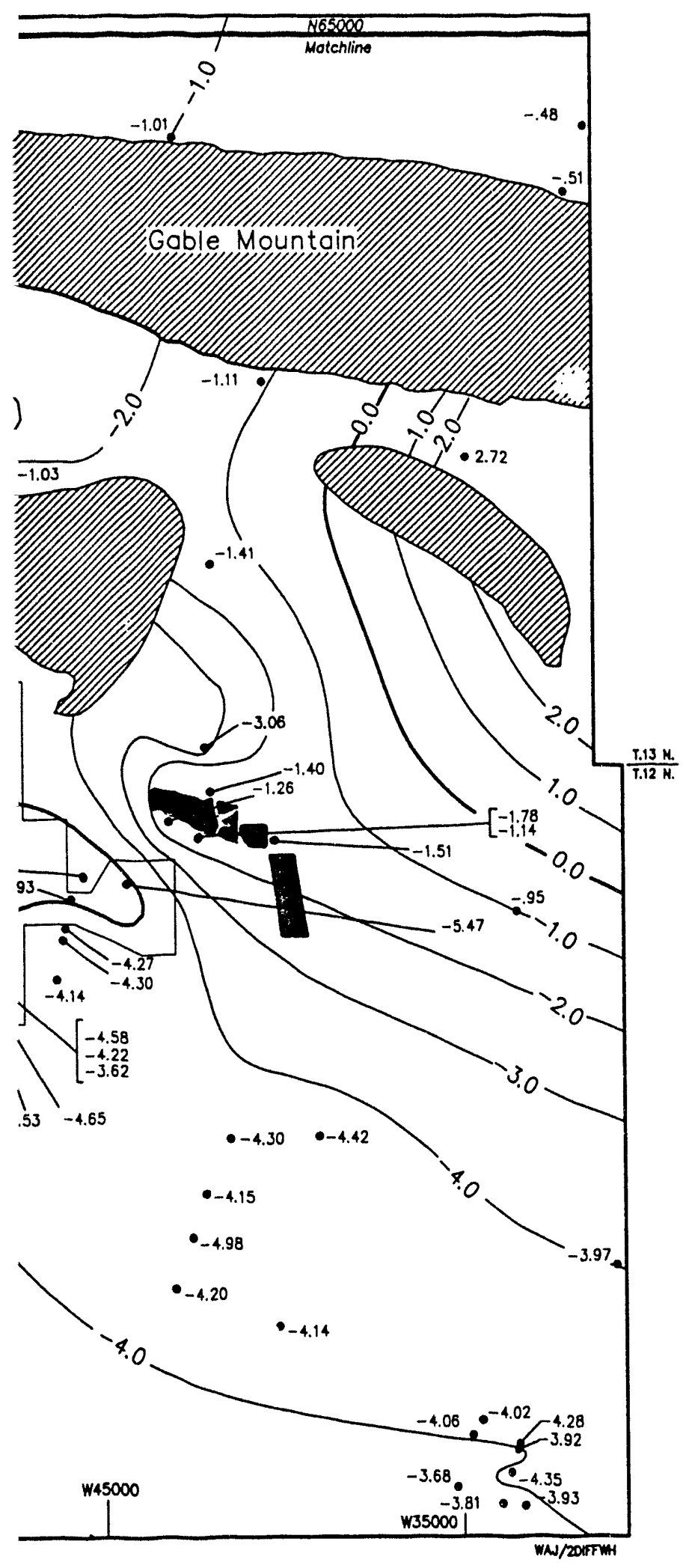

\section{Areas Change in Water Table Elevation 1988-1993}

\author{
-2.92 Change in water toble elevotion in feet \\ measured between December 1988 and \\ December 1993. \\ Groundwoter table elevation change \\ contour interval $=1.0 \mathrm{ft}$. \\ Ponds \\ UIID \\ Areas where the basalt surface is \\ generally above the water table
}

The 200 Areas woter level difference map has been prepared by the Geohydrologic Support Function, Westinghouse Hanford Company.

Note: To convert to metric, multiply elevation ( $\mathrm{ft}$ ) by 0.3048 to obtain elevation $(\mathrm{m})$.
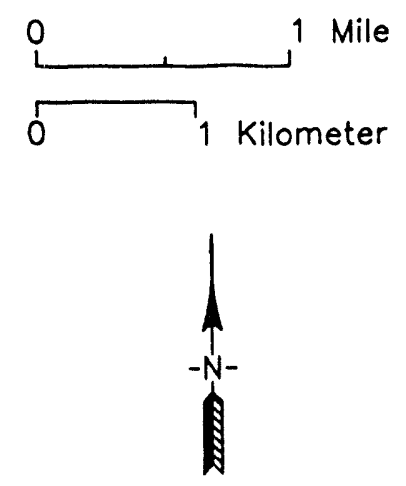


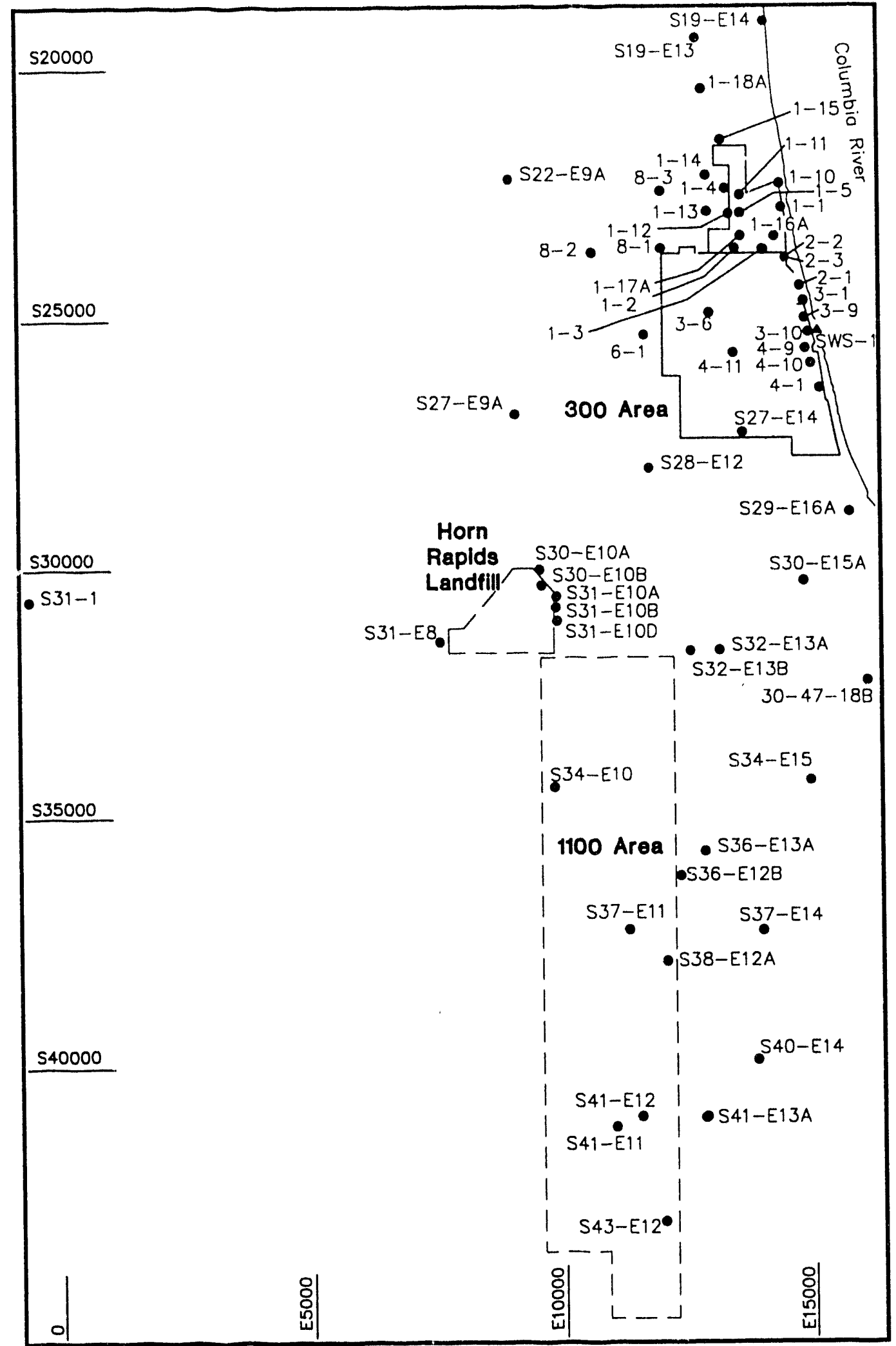

WAJ/310XDC93 
Figure 9

\section{0/1100 Area Index Map December 1993}

3-1 Identification of monitoring well

- used to prepare map. Well Numbers starting with $S$ are prefixed by 699- and well numbers starting with a single digit ore prefixed by 399-.

- River stage recorder

The 300 Area index map has been prepared by the Geohydrologic Support Function, Westinghouse Hanford Company.

Note: To convert to metric, multiply elevation ( $\mathrm{ft}$ ) by 0.3048 to obtain elevation $(\mathrm{m})$.

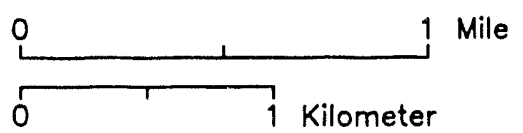




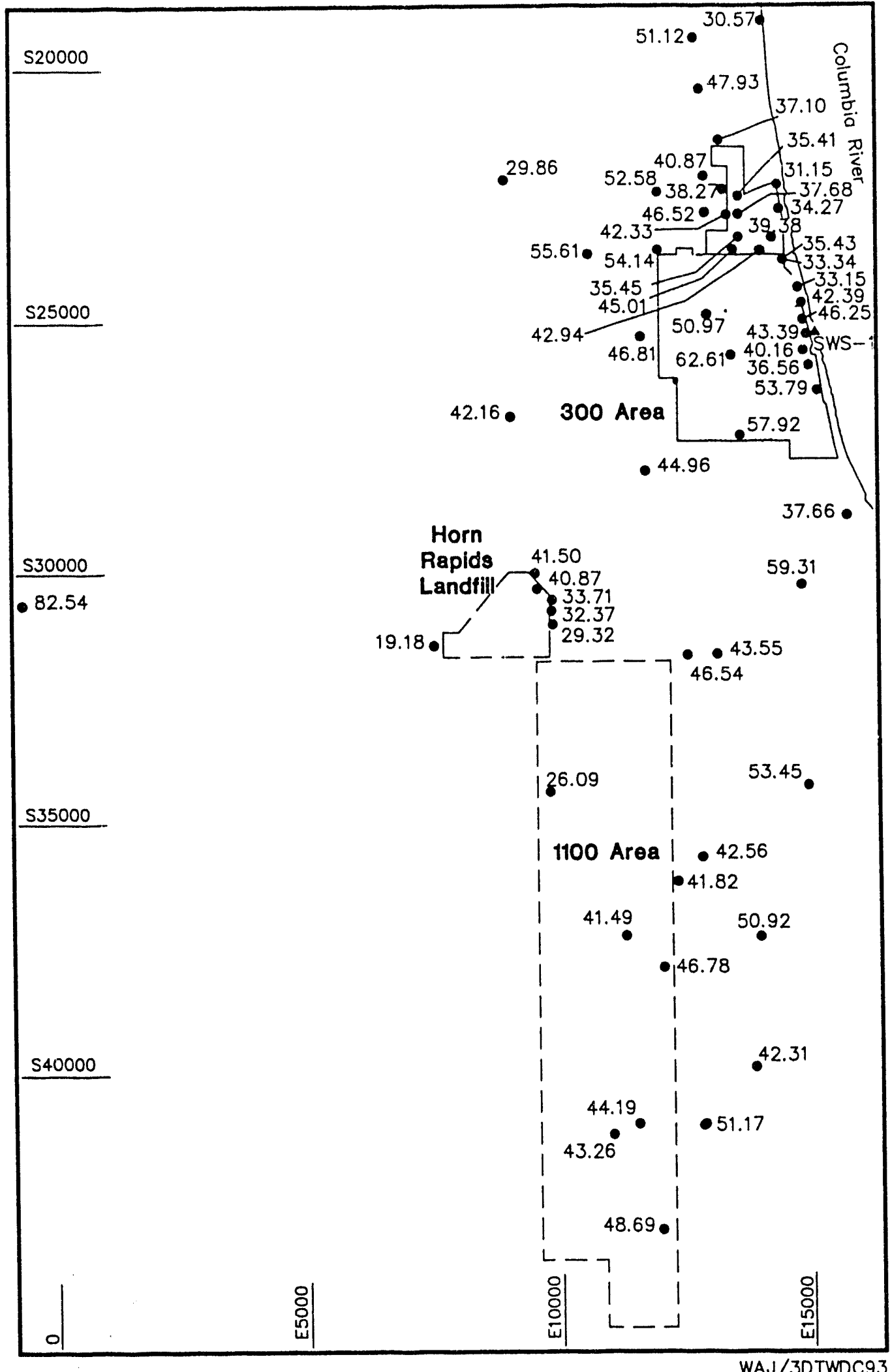

WAJ/3DTWDC93 
WHC-EP-0394-8

Figure 10

\title{
300/1100 Area Depth-to-Water Map December 1993
}

\author{
35.56 Depth to water in feet, as \\ - measured from well reference \\ mark (generally top of casing) \\ to groundwater surface.
}

- River stoge recorder

The 300 Area depth-to-water map has been prepared by the Geohyrologic Support Function, Westinghouse Hanford Company.

Note: To convert to metric, multiply elevation ( $\mathrm{ft}$ ) by 0.3048 to obtain elevation (m).

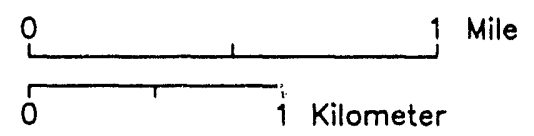




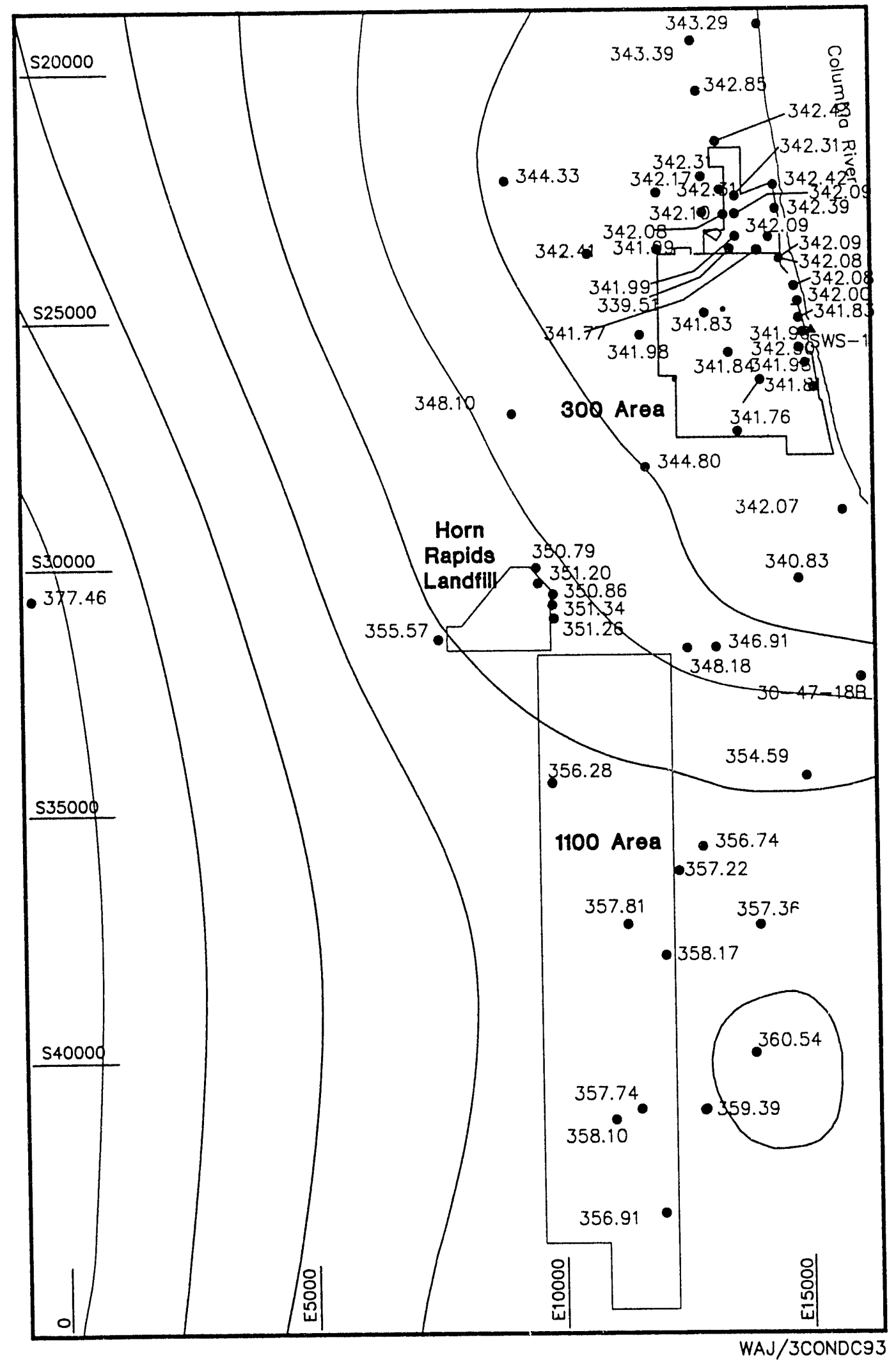


WHC-EP-0394-8

Figure 11

\section{0/1100 Area Water Table Map December 1993}

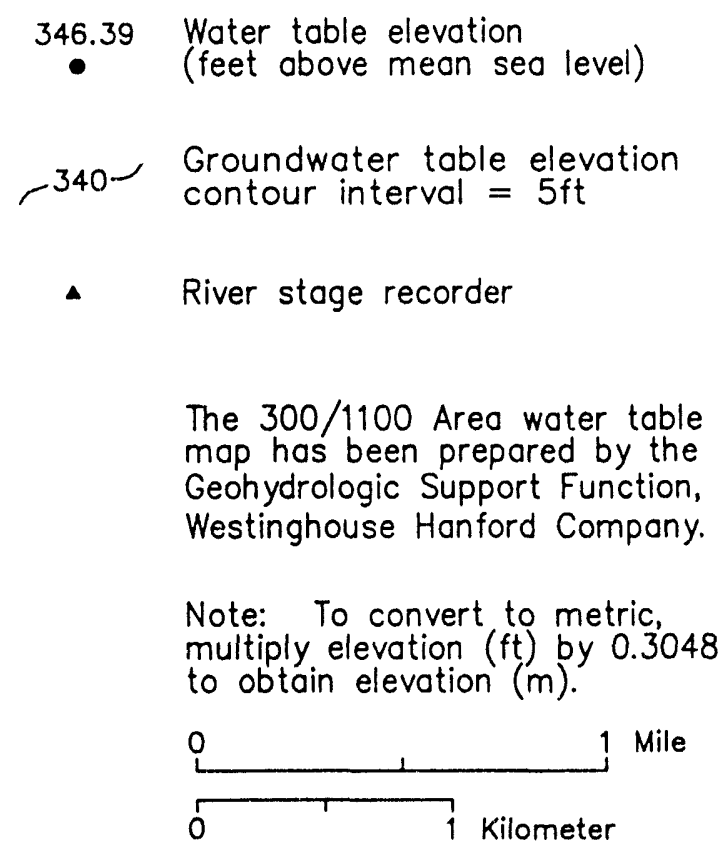

$\overbrace{-N-}^{1}$ 
WHC-EP-0394-8

\section{APPENDIX A}

WATER LEVEL MEASUREMENT DATA

$A-i$ 
WHC-EP-0394-8

Appendix A: December 1993 Water Level Measurement Data (Sheet 1 of 22)

\begin{tabular}{|c|c|c|c|c|}
\hline \multirow{2}{*}{ Well } & \multirow{2}{*}{ Notes } & \multirow{2}{*}{$\begin{array}{l}\text { Depth to } \\
\text { Water, ft }\end{array}$} & \multicolumn{2}{|c|}{ Elevation, ft above msl } \\
\hline & & & Adjusted Casing & Water Level \\
\hline $\begin{array}{l}199-B 2-12 \\
199-B 2-13 \\
199-B 3-1 \\
199-B 3-46 \\
199-B 3-47\end{array}$ & $\begin{array}{l}P \\
P \\
N \\
N\end{array}$ & $\begin{array}{l}45.60 \\
24.63 \\
47.08 \\
49.55 \\
46.22\end{array}$ & $\begin{array}{l}439.06 \\
418.53 \\
439.19 \\
441.62 \\
438.78\end{array}$ & $\begin{array}{l}393.46 \\
393.90 \\
392.11 \\
392.07 \\
392.56\end{array}$ \\
\hline $\begin{array}{l}199-B 4-1 \\
199-B 4-3 \\
199-B 4-4 \\
199-B 4-6 \\
199-B 4-8\end{array}$ & $\begin{array}{l}N \\
N \\
N\end{array}$ & $\begin{array}{l}65.00 \\
65.49 \\
76.98 \\
85.41 \\
76.01\end{array}$ & $\begin{array}{l}461.19 \\
461.71 \\
472.14 \\
481.92 \\
473.58\end{array}$ & $\begin{array}{l}396.19 \\
396.22 \\
395.16 \\
396.51 \\
397.57\end{array}$ \\
\hline $\begin{array}{l}199-B 4-9 \\
199-B 5-1 \\
199-B 5-2 \\
199-B 8-6 \\
199-B 9-1\end{array}$ & $\begin{array}{l}N \\
N \\
Q\end{array}$ & $\begin{array}{l}74.95 \\
59.54 \\
62.94 \\
78.88 \\
97.86\end{array}$ & $\begin{array}{l}471.45 \\
455.58 \\
458.91 \\
475.38 \\
478.87\end{array}$ & $\begin{array}{l}396.50 \\
396.04 \\
395.97 \\
396.50 \\
381.01\end{array}$ \\
\hline $\begin{array}{l}199-B 9-2 \\
199-B 9-3 \\
199-D 2-5 \\
199-D 2-6 \\
199-D 5-12\end{array}$ & $\begin{array}{l}N \\
N\end{array}$ & $\begin{array}{r}100.85 \\
96.48 \\
75.46 \\
85.51 \\
85.66\end{array}$ & $\begin{array}{l}497.42 \\
493.06 \\
460.30 \\
469.28 \\
469.63\end{array}$ & $\begin{array}{l}396.57 \\
396.58 \\
384.84 \\
383.77 \\
383.97\end{array}$ \\
\hline $\begin{array}{l}199-D 5-13 \\
199-D 5-14 \\
199-D 5-15 \\
199-D 5-16 \\
199-D 5-17\end{array}$ & $\begin{array}{l}N \\
N \\
N \\
N \\
N\end{array}$ & $\begin{array}{l}85.68 \\
88.03 \\
87.58 \\
89.25 \\
85.00\end{array}$ & $\begin{array}{l}471.49 \\
471.69 \\
471.53 \\
472.89 \\
469.49\end{array}$ & $\begin{array}{l}385.81 \\
383.66 \\
383.95 \\
383.64 \\
384.49\end{array}$ \\
\hline $\begin{array}{l}199-D 5-18 \\
199-D 5-19 \\
199-D 5-20 \\
199-D 8-3 \\
199-D 8-4\end{array}$ & $\begin{array}{l}N \\
N \\
N \\
N\end{array}$ & $\begin{array}{l}82.49 \\
80.25 \\
85.25 \\
68.92 \\
85.26\end{array}$ & $\begin{array}{l}466.68 \\
464.80 \\
468.10 \\
448.99 \\
468.73\end{array}$ & $\begin{array}{l}384.19 \\
384.55 \\
382.85 \\
380.07 \\
383.47\end{array}$ \\
\hline $\begin{array}{l}199-D 8-5 \\
199-D 8-53 \\
199-D 8-54 A \\
199-D 8-54 B \\
199-D 8-55\end{array}$ & $\begin{array}{l}N \\
N \\
P \\
N\end{array}$ & $\begin{array}{l}70.42 \\
54.97 \\
61.69 \\
60.98 \\
58.09\end{array}$ & $\begin{array}{l}452.49 \\
436.03 \\
442.78 \\
442.51 \\
439.35\end{array}$ & $\begin{array}{l}382.07 \\
381.06 \\
381.09 \\
381.53 \\
381.26\end{array}$ \\
\hline
\end{tabular}


Appendix A: December 1993 Water Level Measurement Data (Sheet ? of 22)

\begin{tabular}{|c|c|c|c|c|}
\hline \multirow{2}{*}{ Well } & \multirow{2}{*}{ Notes } & \multirow{2}{*}{$\begin{array}{l}\text { Depth to } \\
\text { Water, ft }\end{array}$} & \multicolumn{2}{|c|}{ Elevation, $\mathrm{ft}$ above $\mathrm{msl}$} \\
\hline & & & Adjusted Casing & Water Level \\
\hline $\begin{array}{l}199-38-6 \\
199-F 1-2 \\
199-F 5-1 \\
199-F 5-3 \\
199-F 5-4\end{array}$ & $\begin{array}{l}Q \\
N\end{array}$ & $\begin{array}{l}93.02 \\
28.24 \\
36.96 \\
55.76 \\
42.66\end{array}$ & $\begin{array}{l}476.50 \\
397.95 \\
406.56 \\
408.62 \\
412.12\end{array}$ & $\begin{array}{l}383.48 \\
369.71 \\
369.60 \\
352.86 \\
369.46\end{array}$ \\
\hline $\begin{array}{l}199-F 5-42 \\
199-F 5-43 A \\
199-F 5-43 B \\
199-F 5-44 \\
199-F 5-45\end{array}$ & $\begin{array}{l}N \\
N \\
P \\
N \\
N\end{array}$ & $\begin{array}{l}22.53 \\
26.18 \\
26.13 \\
33.48 \\
45.56\end{array}$ & $\begin{array}{l}391.26 \\
395.35 \\
394.87 \\
402.65 \\
414.31\end{array}$ & $\begin{array}{l}368.73 \\
369.17 \\
368.74 \\
369.17 \\
368.75\end{array}$ \\
\hline $\begin{array}{l}199-F 5-46 \\
199-F 5-47 \\
199-F 5-48 \\
199-F 5-6 \\
199-F 6-1\end{array}$ & $\begin{array}{l}N \\
N \\
N \\
N \\
N\end{array}$ & $\begin{array}{l}48.49 \\
49.23 \\
48.15 \\
44.69 \\
37.70\end{array}$ & $\begin{array}{l}416.95 \\
419.03 \\
417.36 \\
412.95 \\
405.15\end{array}$ & $\begin{array}{l}368.46 \\
369.80 \\
369.21 \\
368.26 \\
367.45\end{array}$ \\
\hline $\begin{array}{l}199-F 7-1 \\
199-F 7-2 \\
199-F 7-3 \\
199-F 8-1 \\
199-F 8-2\end{array}$ & $\begin{array}{l}Q \\
N \\
N\end{array}$ & $\begin{array}{l}17.46 \\
23.65 \\
22.09 \\
35.45 \\
40.52\end{array}$ & $\begin{array}{l}389.74 \\
410.72 \\
394.84 \\
405.86 \\
410.74\end{array}$ & $\begin{array}{l}372.28 \\
387.07 \\
372.75 \\
370.41 \\
370.22\end{array}$ \\
\hline $\begin{array}{l}199-F 8-3 \\
199-F 8-4 \\
199-H 3-1 \\
199-H 3-2 A \\
199-H 3-2 B\end{array}$ & $\begin{array}{l}N \\
N \\
N \\
N\end{array}$ & $\begin{array}{l}28.27 \\
44.01 \\
46.12 \\
42.89 \\
43.48\end{array}$ & $\begin{array}{l}399.71 \\
410.93 \\
421.48 \\
417.83 \\
418.42\end{array}$ & $\begin{array}{l}371.44 \\
366.92 \\
375.36 \\
374.94 \\
374.94\end{array}$ \\
\hline $\begin{array}{l}199-H 3-2 C \\
199-H 4-10 \\
199-H 4-11 \\
199-H 4-12 A \\
199-H 4-12 B\end{array}$ & $\begin{array}{l}P \\
N \\
N \\
N \\
N\end{array}$ & $\begin{array}{l}43.41 \\
30.29 \\
44.16 \\
40.36 \\
40.38\end{array}$ & $\begin{array}{l}418.22 \\
404.44 \\
416.84 \\
413.50 \\
413.52\end{array}$ & $\begin{array}{l}374.81 \\
374.15 \\
372.68 \\
373.14 \\
373.14\end{array}$ \\
\hline $\begin{array}{l}199-H 4-12 C \\
199-H 4-13 \\
199-H 4-14 \\
199-H 4-15 A \\
199-H 4-15 B\end{array}$ & $\begin{array}{l}P \\
N \\
N \\
N \\
N\end{array}$ & $\begin{array}{l}40.69 \\
45.99 \\
45.95 \\
33.91 \\
33.62\end{array}$ & $\begin{array}{l}413.52 \\
418.20 \\
420.59 \\
407.21 \\
406.92\end{array}$ & $\begin{array}{l}372.83 \\
372.21 \\
374.64 \\
373.30 \\
373.30\end{array}$ \\
\hline
\end{tabular}


Appendix A: December 1993 Water Level Measurement Data (Sheet 3 of 22)

\begin{tabular}{|c|c|c|c|c|}
\hline \multirow{2}{*}{ Well } & \multirow{2}{*}{ Notes } & \multirow{2}{*}{$\begin{array}{l}\text { Depth to } \\
\text { Water, ft }\end{array}$} & \multicolumn{2}{|c|}{ Elevation, ft above msl } \\
\hline & & & Adjusted Casing & Water Level \\
\hline $\begin{array}{l}199-H 4-15 C R \\
199-H 4-15 C S \\
199-H 4-16 \\
199-H 4-17 \\
199-H 4-18\end{array}$ & $\begin{array}{l}P \\
P \\
N \\
N\end{array}$ & $\begin{array}{l}33.88 \\
34.27 \\
50.16 \\
45.37 \\
48.13\end{array}$ & $\begin{array}{l}407.37 \\
407.44 \\
424.23 \\
419.09 \\
421.82\end{array}$ & $\begin{array}{l}373.49 \\
373.17 \\
374.07 \\
373.72 \\
373.69\end{array}$ \\
\hline $\begin{array}{l}199-\mathrm{H} 4-3 \\
199-\mathrm{H} 4-4 \\
199-\mathrm{H} 4-45 \\
199-\mathrm{H} 4-46 \\
199-\mathrm{H} 4-47\end{array}$ & $\begin{array}{l}N \\
N \\
N \\
N \\
N\end{array}$ & $\begin{array}{l}46.72 \\
40.75 \\
42.90 \\
49.31 \\
49.82\end{array}$ & $\begin{array}{l}420.29 \\
413.70 \\
416.64 \\
424.19 \\
424.91\end{array}$ & $\begin{array}{l}373.57 \\
372.95 \\
373.74 \\
374.88 \\
375.09\end{array}$ \\
\hline $\begin{array}{l}199-H 4-48 \\
199-H 4-49 \\
199-H 4-5 \\
199-H 4-6 \\
199-H 4-7\end{array}$ & $\begin{array}{l}N \\
N \\
N \\
N \\
N\end{array}$ & $\begin{array}{l}50.68 \\
48.99 \\
42.98 \\
44.65 \\
46.43\end{array}$ & $\begin{array}{l}426.02 \\
424.85 \\
416.21 \\
419.58 \\
420.59\end{array}$ & $\begin{array}{l}375.34 \\
375.86 \\
373.23 \\
374.93 \\
374.16\end{array}$ \\
\hline $\begin{array}{l}199-H 4-8 \\
199-H 4-9 \\
199-H 5-1 \\
199-H 6-1 \\
199-K-11\end{array}$ & $\begin{array}{l}\mathbf{N} \\
\mathbf{N}\end{array}$ & $\begin{array}{l}46.19 \\
44.54 \\
44.26 \\
44.36 \\
75.51\end{array}$ & $\begin{array}{l}420.00 \\
418.08 \\
420.16 \\
418.10 \\
466.55\end{array}$ & $\begin{array}{l}373.81 \\
373.54 \\
375.90 \\
373.74 \\
391.04\end{array}$ \\
\hline $\begin{array}{l}199-K-13 \\
199-K-18 \\
199-K-19 \\
199-K-20 \\
199-K-21\end{array}$ & $\begin{array}{l}N \\
N\end{array}$ & $\begin{array}{l}74.40 \\
23.42 \\
35.15 \\
35.83 \\
36.38\end{array}$ & $\begin{array}{l}464.00 \\
409.00 \\
422.17 \\
422.57 \\
421.73\end{array}$ & $\begin{array}{l}389.60 \\
385.58 \\
387.02 \\
386.74 \\
385.35\end{array}$ \\
\hline $\begin{array}{l}199-K-22 \\
199-K-23 \\
199-K-27 \\
199-K-28 \\
199-K-30\end{array}$ & $\begin{array}{l}N \\
Q \\
N \\
N\end{array}$ & $\begin{array}{l}38.89 \\
75.49 \\
74.23 \\
73.56 \\
73.59\end{array}$ & $\begin{array}{l}424.51 \\
405.00 \\
466.67 \\
465.97 \\
466.20\end{array}$ & $\begin{array}{l}385.62 \\
329.51 \\
392.44 \\
392.41 \\
392.61\end{array}$ \\
\hline $\begin{array}{l}199-K-31 \\
199-K-32 A \\
199-K-32 B \\
199-K-33 \\
199-K-34\end{array}$ & $\begin{array}{l}N \\
N \\
P \\
N\end{array}$ & $\begin{array}{l}26.03 \\
55.89 \\
48.82 \\
56.82 \\
77.99\end{array}$ & $\begin{array}{l}412.40 \\
444.02 \\
445.27 \\
443.64 \\
468.09\end{array}$ & $\begin{array}{l}386.37 \\
388.13 \\
396.45 \\
386.82 \\
390.10\end{array}$ \\
\hline
\end{tabular}


Appendix A: December 1993 Water Level Measurement Data (Sheet 4 of 22)

\begin{tabular}{|c|c|c|c|c|}
\hline \multirow{2}{*}{ Well } & \multirow{2}{*}{ Notes } & \multirow{2}{*}{$\begin{array}{l}\text { Depth to } \\
\text { Water, ft }\end{array}$} & \multicolumn{2}{|c|}{ Elevation, ft above msl } \\
\hline & & & Adjusted Casing & Water Level \\
\hline $\begin{array}{l}199-K-35 \\
199-K-36 \\
199-K-37 \\
199-N-14 \\
199-N-16\end{array}$ & N & $\begin{array}{l}99.56 \\
98.41 \\
55.43 \\
69.58 \\
71.28\end{array}$ & $\begin{array}{l}494.55 \\
494.07 \\
441.80 \\
453.68 \\
457.24\end{array}$ & $\begin{array}{l}394.99 \\
395.66 \\
386.37 \\
384.10 \\
385.96\end{array}$ \\
\hline $\begin{array}{l}199-N-17 \\
199-N-19 \\
199-N-2 \\
199-N-20 \\
199-N-21\end{array}$ & $\begin{array}{l}N \\
N \\
N \\
N \\
N\end{array}$ & $\begin{array}{l}76.68 \\
69.80 \\
75.13 \\
71.40 \\
72.56\end{array}$ & $\begin{array}{l}461.71 \\
454.44 \\
459.76 \\
456.35 \\
457.47\end{array}$ & $\begin{array}{l}385.03 \\
384.64 \\
384.63 \\
384.95 \\
384.91\end{array}$ \\
\hline $\begin{array}{l}199-N-23 \\
199-N-25 \\
199-N-26 \\
199-N-27 \\
199-N-28\end{array}$ & $\begin{array}{l}N \\
N \\
N \\
N\end{array}$ & $\begin{array}{l}72.05 \\
41.85 \\
71.69 \\
62.41 \\
76.84\end{array}$ & $\begin{array}{l}456.80 \\
426.25 \\
456.26 \\
449.60 \\
464.74\end{array}$ & $\begin{array}{l}384.75 \\
384.40 \\
384.57 \\
387.19 \\
387.90\end{array}$ \\
\hline $\begin{array}{l}199-N-29 \\
199-N-3 \\
199-N-31 \\
199-N-32 \\
199-N-33\end{array}$ & $\begin{array}{l}N \\
N \\
N \\
N \\
N\end{array}$ & $\begin{array}{l}77.80 \\
75.69 \\
76.58 \\
75.87 \\
75.60\end{array}$ & $\begin{array}{l}465.75 \\
459.39 \\
462.63 \\
462.59 \\
460.37\end{array}$ & $\begin{array}{l}387.95 \\
383.70 \\
386.05 \\
386.72 \\
384.77\end{array}$ \\
\hline $\begin{array}{l}199-N-34 \\
199-N-41 \\
199-N-42 \\
199-N-50 \\
199-N-51\end{array}$ & $\begin{array}{l}N \\
N \\
N \\
N \\
N\end{array}$ & $\begin{array}{l}73.29 \\
73.49 \\
70.77 \\
79.93 \\
78.95\end{array}$ & $\begin{array}{l}460.15 \\
458.11 \\
455.64 \\
463.90 \\
462.72\end{array}$ & $\begin{array}{l}386.86 \\
384.62 \\
384.87 \\
383.97 \\
383.77\end{array}$ \\
\hline $\begin{array}{l}199-N-52 \\
199-N-54 \\
199-N-55 \\
199-N-56 \\
199-N-57\end{array}$ & $\begin{array}{l}N \\
N \\
N \\
N \\
N\end{array}$ & $\begin{array}{l}76.16 \\
72.49 \\
72.81 \\
73.48 \\
71.50\end{array}$ & $\begin{array}{l}464.20 \\
458.00 \\
458.35 \\
458.59 \\
458.26\end{array}$ & $\begin{array}{l}388.04 \\
385.51 \\
385.54 \\
385.11 \\
386.76\end{array}$ \\
\hline $\begin{array}{l}199-N-59 \\
199-N-62 \\
199-N-63 \\
199-N-64 \\
199-N-65\end{array}$ & $\begin{array}{l}N \\
N \\
N \\
N\end{array}$ & $\begin{array}{l}74.99 \\
76.21 \\
79.66 \\
68.02 \\
70.34\end{array}$ & $\begin{array}{l}460.07 \\
464.12 \\
467.23 \\
455.15 \\
456.98\end{array}$ & $\begin{array}{l}385.08 \\
387.91 \\
387.57 \\
387.13 \\
386.64\end{array}$ \\
\hline
\end{tabular}


Appendix A: December 1993 Water Level Measurement Data (Sheet 5 of 22)

\begin{tabular}{|c|c|c|c|c|}
\hline \multirow{2}{*}{ Well } & \multirow{2}{*}{ Notes } & \multirow{2}{*}{$\begin{array}{l}\text { Depth to } \\
\text { Water, ft }\end{array}$} & \multicolumn{2}{|c|}{ Elevation, ft above ms 1} \\
\hline & & & Adjusted Casing & Water Leve1 \\
\hline $\begin{array}{l}199-N-67 \\
199-N-69 \\
199-N-70 \\
199-N-71 \\
199-N-72\end{array}$ & $\begin{array}{l}N \\
N \\
N \\
N \\
N\end{array}$ & $\begin{array}{l}73.96 \\
74.45 \\
69.14 \\
73.73 \\
70.60\end{array}$ & $\begin{array}{l}458.97 \\
459.36 \\
454.70 \\
463.02 \\
458.98\end{array}$ & $\begin{array}{l}385.01 \\
384.91 \\
385.56 \\
389.29 \\
388.38\end{array}$ \\
\hline $\begin{array}{l}199-N-73 \\
199-N-74 \\
199-N-75 \\
199-N-76 \\
199-N-77\end{array}$ & $\begin{array}{l}N \\
N \\
N \\
N\end{array}$ & $\begin{array}{l}75.64 \\
68.55 \\
72.17 \\
68.94 \\
71.25\end{array}$ & $\begin{array}{l}463.26 \\
457.64 \\
456.78 \\
452.12 \\
459.44\end{array}$ & $\begin{array}{l}387.62 \\
389.09 \\
384.61 \\
383.18 \\
388.19\end{array}$ \\
\hline $\begin{array}{l}199-N-80 \\
199-N-81 \\
199-N-8 S \\
299-E 13-10 \\
299-E 13-12\end{array}$ & $\begin{array}{l}P \\
N\end{array}$ & $\begin{array}{r}73.94 \\
77.00 \\
21.48 \\
337.05 \\
331.38\end{array}$ & $\begin{array}{l}457.69 \\
462.71 \\
405.12 \\
738.84 \\
731.34\end{array}$ & $\begin{array}{l}383.75 \\
385.71 \\
383.64 \\
401.79 \\
399.96\end{array}$ \\
\hline $\begin{array}{l}299-E 13-14 \\
299-E 16-1 \\
299-E 17-1 \\
299-E 17-10 \\
299-E 17-12\end{array}$ & $Q$ & $\begin{array}{l}343.41 \\
290.19 \\
317.60 \\
313.24 \\
320.63\end{array}$ & $\begin{array}{l}745.15 \\
696.44 \\
719.17 \\
714.74 \\
721.70\end{array}$ & $\begin{array}{l}401.74 \\
406.25 \\
401.57 \\
401.50 \\
401.07\end{array}$ \\
\hline $\begin{array}{l}299-\mathrm{E} 17-13 \\
299-\mathrm{E} 17-14 \\
299-\mathrm{E} 17-15 \\
299-\mathrm{E} 17-16 \\
299-\mathrm{E} 17-17\end{array}$ & $N$ & $\begin{array}{l}317.92 \\
320.74 \\
321.16 \\
319.36 \\
318.58\end{array}$ & $\begin{array}{l}719.25 \\
722.18 \\
721.78 \\
720.58 \\
719.92\end{array}$ & $\begin{array}{l}401.33 \\
401.44 \\
400.62 \\
401.22 \\
401.34\end{array}$ \\
\hline $\begin{array}{l}299-E 17-18 \\
299-E 17-19 \\
299-E 17-20 \\
299-E 17-5 \\
299-E 17-6\end{array}$ & & $\begin{array}{l}319.29 \\
318.68 \\
317.65 \\
317.74 \\
318.68\end{array}$ & $\begin{array}{l}720.65 \\
719.33 \\
719.23 \\
718.69 \\
720.10\end{array}$ & $\begin{array}{l}401.36 \\
400.65 \\
401.58 \\
400.95 \\
401.42\end{array}$ \\
\hline $\begin{array}{l}299-E 17-9 \\
299-E 18-1 \\
299-E 18-2 \\
299-E 18-3 \\
299-E 18-4\end{array}$ & $\begin{array}{l}N \\
N\end{array}$ & $\begin{array}{l}316.44 \\
318.35 \\
319.62 \\
320.72 \\
319.72\end{array}$ & $\begin{array}{l}717.64 \\
720.24 \\
721.21 \\
722.04 \\
721.57\end{array}$ & $\begin{array}{l}401.20 \\
401.89 \\
401.59 \\
401.32 \\
401.85\end{array}$ \\
\hline
\end{tabular}


Appendix A: December 1993 Water Level Measurement Data (Sheet 6 of 22)

\begin{tabular}{|c|c|c|c|c|}
\hline \multirow{2}{*}{ Well } & \multirow{2}{*}{ Notes } & \multirow{2}{*}{$\begin{array}{l}\text { Depth to } \\
\text { Water, ft }\end{array}$} & \multicolumn{2}{|c|}{ Elevation, ft above msl } \\
\hline & & & Adjusted Casing & Water Level \\
\hline $\begin{array}{l}299-E 23-1 \\
299-E 23-2 \\
299-E 24-18 \\
299-E 24-19 \\
299-E 24-2\end{array}$ & $N$ & $\begin{array}{l}311.57 \\
319.37 \\
316.96 \\
292.12 \\
315.51\end{array}$ & $\begin{array}{l}713.10 \\
720.64 \\
719.28 \\
693.65 \\
717.47\end{array}$ & $\begin{array}{l}401.53 \\
401.27 \\
402.32 \\
401.53 \\
401.96\end{array}$ \\
\hline $\begin{array}{l}299-E 24-20 \\
299-E 24-7 \\
299-E 24-8 \\
299-E 25-10 \\
299-E 25-11\end{array}$ & $N$ & $\begin{array}{l}287.46 \\
314.72 \\
286.85 \\
253.87 \\
279.35\end{array}$ & $\begin{array}{l}689.28 \\
716.01 \\
688.81 \\
655.84 \\
681.28\end{array}$ & $\begin{array}{l}401.82 \\
401.29 \\
401.96 \\
401.97 \\
401.93\end{array}$ \\
\hline $\begin{array}{l}299-E 25-18 \\
299-E 25-19 \\
299-E 25-2 \\
299-E 25-20 \\
299-E 25-21\end{array}$ & N & $\begin{array}{l}277.10 \\
275.29 \\
273.71 \\
274.43 \\
275.56\end{array}$ & $\begin{array}{l}679.05 \\
677.20 \\
675.45 \\
676.30 \\
677.27\end{array}$ & $\begin{array}{l}401.95 \\
401.91 \\
401.74 \\
401.87 \\
401.71\end{array}$ \\
\hline $\begin{array}{l}299-E 25-26 \\
299-E 25-28 \\
299-E 25-32 P \\
299-E 25-32 Q \\
299-E 25-34\end{array}$ & & $\begin{array}{l}266.40 \\
260.30 \\
267.98 \\
268.32 \\
260.83\end{array}$ & $\begin{array}{l}668.52 \\
662.44 \\
670.04 \\
670.04 \\
662.87\end{array}$ & $\begin{array}{l}402.12 \\
402.14 \\
402.06 \\
401.72 \\
402.04\end{array}$ \\
\hline $\begin{array}{l}299-E 25-35 \\
299-E 25-36 \\
299-E 25-40 \\
299-E 25-41 \\
299-E 25-42\end{array}$ & $N$ & $\begin{array}{l}273.02 \\
305.30 \\
264.03 \\
269.53 \\
281.47\end{array}$ & $\begin{array}{l}674.39 \\
707.39 \\
665.71 \\
671.26 \\
683.29\end{array}$ & $\begin{array}{l}401.37 \\
402.09 \\
401.68 \\
401.73 \\
401.82\end{array}$ \\
\hline $\begin{array}{l}299-E 25-43 \\
299-E 25-46 \\
299-E 25-47 \\
299-E 25-48 \\
299-E 25-6\end{array}$ & $\begin{array}{l}N \\
N \\
N \\
N \\
Q\end{array}$ & $\begin{array}{l}247.85 \\
292.92 \\
271.66 \\
280.25 \\
258.99\end{array}$ & $\begin{array}{l}649.89 \\
694.81 \\
673.77 \\
682.31 \\
658.31\end{array}$ & $\begin{array}{l}402.04 \\
401.89 \\
402.11 \\
402.06 \\
399.32\end{array}$ \\
\hline $\begin{array}{l}299-E 25-9 \\
299-E 26-1 \\
299-E 26-10 \\
299-E 26-12 \\
299-E 26-13\end{array}$ & $\begin{array}{l}Q \\
N \\
N \\
N\end{array}$ & $\begin{array}{l}255.02 \\
214.96 \\
199.28 \\
228.28 \\
202.59\end{array}$ & $\begin{array}{l}654.86 \\
617.25 \\
601.47 \\
630.74 \\
605.02\end{array}$ & $\begin{array}{l}399.84 \\
402.29 \\
402.19 \\
402.46 \\
402.43\end{array}$ \\
\hline
\end{tabular}


Appendix A: December 1993 Water Level Measurement Data (Sheet 7 of 22)

\begin{tabular}{|c|c|c|c|c|}
\hline \multirow{2}{*}{ Well } & \multirow{2}{*}{ Notes } & \multirow{2}{*}{$\begin{array}{l}\text { Depth to } \\
\text { Water, ft }\end{array}$} & \multicolumn{2}{|c|}{ Elevation, $\mathrm{ft}$ above $\mathrm{ms} 1$} \\
\hline & & & Adjusted Casing & Water Level \\
\hline $\begin{array}{l}299-E 26-2 \\
299-E 26-4 \\
299-E 26-8 \\
299-E 26-9 \\
299-E 27-1\end{array}$ & $\begin{array}{l}Q \\
N \\
N\end{array}$ & $\begin{array}{l}233.00 \\
245.94 \\
217.81 \\
200.71 \\
280.97\end{array}$ & $\begin{array}{l}635.30 \\
647.76 \\
602.76 \\
602.89 \\
682.55\end{array}$ & $\begin{array}{l}402.30 \\
401.82 \\
384.95 \\
402.18 \\
401.58\end{array}$ \\
\hline $\begin{array}{l}299-E 27-10 \\
299-E 2 T-11 \\
299-E 27-12 \\
299-E 27-13 \\
299-E 27-14\end{array}$ & & $\begin{array}{l}222.51 \\
241.79 \\
259.38 \\
267.24 \\
256.74\end{array}$ & $\begin{array}{l}624.47 \\
643.29 \\
660.95 \\
668.99 \\
658.34\end{array}$ & $\begin{array}{l}401.96 \\
401.50 \\
401.57 \\
401.75 \\
401.60\end{array}$ \\
\hline $\begin{array}{l}299-E 27-15 \\
299-E 27-17 \\
299-E 27-7 \\
299-E 27-8 \\
299-E 27-9\end{array}$ & $N$ & $\begin{array}{l}251.97 \\
232.86 \\
232.94 \\
236.19 \\
227.47\end{array}$ & $\begin{array}{l}652.67 \\
634.72 \\
634.67 \\
637.83 \\
629.21\end{array}$ & $\begin{array}{l}400.70 \\
401.86 \\
401.73 \\
401.64 \\
401.74\end{array}$ \\
\hline $\begin{array}{l}299-E 28-12 \\
299-E 28-17 \\
299-E 28-18 \\
299-E 28-26 \\
299-E 28-27\end{array}$ & & $\begin{array}{l}306.62 \\
306.12 \\
290.93 \\
285.81 \\
279.01\end{array}$ & $\begin{array}{l}708.60 \\
708.56 \\
692.58 \\
687.26 \\
680.37\end{array}$ & $\begin{array}{l}401.98 \\
402.44 \\
401.65 \\
401.45 \\
401.36\end{array}$ \\
\hline $\begin{array}{l}299-E 28-28 \\
299-E 28-4 \\
299-E 28-6 \\
299-E 28-7 \\
299-E 28-9\end{array}$ & & $\begin{array}{l}285.28 \\
289.86 \\
298.48 \\
285.07 \\
299.20\end{array}$ & $\begin{array}{l}686.55 \\
691.55 \\
700.11 \\
685.91 \\
700.77\end{array}$ & $\begin{array}{l}401.27 \\
401.69 \\
401.63 \\
400.84 \\
401.57\end{array}$ \\
\hline $\begin{array}{l}299-E 32-1 \\
299-E 32-10 \\
299-E 32-2 \\
299-E 32-3 \\
299-E 32-4\end{array}$ & & $\begin{array}{l}254.60 \\
236.37 \\
268.86 \\
275.15 \\
284.69\end{array}$ & $\begin{array}{l}656.17 \\
637.88 \\
670.06 \\
676.51 \\
685.88\end{array}$ & $\begin{array}{l}401.57 \\
401.51 \\
401.20 \\
401.36 \\
401.19\end{array}$ \\
\hline $\begin{array}{l}299-E 32-5 \\
299-E 32-6 \\
299-E 32-7 \\
299-E 32-8 \\
299-E 32-9\end{array}$ & $\begin{array}{l}\mathbf{N} \\
N \\
N \\
N\end{array}$ & $\begin{array}{l}280.81 \\
265.88 \\
256.87 \\
244.13 \\
241.78\end{array}$ & $\begin{array}{l}682.14 \\
667.45 \\
658.42 \\
645.59 \\
643.33\end{array}$ & $\begin{array}{l}401.33 \\
401.57 \\
401.55 \\
401.46 \\
401.55\end{array}$ \\
\hline
\end{tabular}


Appendix A: December 1993 Water Level Measurement Data (Sheet 8 of 22)

\begin{tabular}{|c|c|c|c|c|}
\hline \multirow{2}{*}{ We11 } & \multirow{2}{*}{ Notes } & \multirow{2}{*}{$\begin{array}{l}\text { Depth to } \\
\text { Water, ft }\end{array}$} & \multicolumn{2}{|c|}{ Elevation, $\mathrm{ft}$ above $\mathrm{ms} 1$} \\
\hline & & & Adjusted Casing & Water Leve1 \\
\hline $\begin{array}{l}299-E 33-1 \\
299-E 33-12 \\
299-E 33-13 \\
299-E 33-14 \\
299-E 33-15\end{array}$ & $\begin{array}{l}\mathbf{N} \\
\mathbf{P} \\
\mathbf{Q}\end{array}$ & $\begin{array}{l}230.42 \\
221.27 \\
226.63 \\
220.31 \\
225.53\end{array}$ & $\begin{array}{l}632.11 \\
623.45 \\
625.53 \\
022.05 \\
627.29\end{array}$ & $\begin{array}{l}401.69 \\
402.18 \\
398.90 \\
401.74 \\
401.76\end{array}$ \\
\hline $\begin{array}{l}299-E 33-17 \\
299-E 33-18 \\
299-E 33-2 \\
299-E 33-21 \\
299-E 33-28\end{array}$ & $\begin{array}{l}N \\
N \\
Q\end{array}$ & $\begin{array}{l}230.05 \\
250.13 \\
230.02 \\
266.64 \\
262.78\end{array}$ & $\begin{array}{l}631.65 \\
651.86 \\
629.75 \\
668.13 \\
664.23\end{array}$ & $\begin{array}{l}401.60 \\
401.73 \\
399.73 \\
401.49 \\
401.45\end{array}$ \\
\hline $\begin{array}{l}299-E 33-29 \\
299-E 33-3 \\
299-E 33-30 \\
299-E 33-31 \\
299-E 33-32\end{array}$ & $Q$ & $\begin{array}{l}272.32 \\
228.87 \\
262.28 \\
245.82 \\
258.56\end{array}$ & $\begin{array}{l}673.77 \\
625.70 \\
663.70 \\
647.28 \\
659.83\end{array}$ & $\begin{array}{l}401.45 \\
396.83 \\
401.42 \\
401.46 \\
401.27\end{array}$ \\
\hline $\begin{array}{l}299-E 33-33 \\
299-E 33-34 \\
299-E 33-35 \\
299-E 33-36 \\
299-E 33-37\end{array}$ & & $\begin{array}{l}238.45 \\
232.05 \\
241.72 \\
246.13 \\
251.17\end{array}$ & $\begin{array}{l}640.17 \\
633.33 \\
643.01 \\
646.67 \\
653.01\end{array}$ & $\begin{array}{l}401.72 \\
401.28 \\
401.29 \\
400.54 \\
401.84\end{array}$ \\
\hline $\begin{array}{l}299-E 33-38 \\
299-E 33-39 \\
299-E 33-4 \\
299-E 33-40 \\
299-E 33-41\end{array}$ & $\begin{array}{l}N \\
N \\
Q \\
N \\
N\end{array}$ & $\begin{array}{l}230.42 \\
221.93 \\
228.11 \\
222.57 \\
253.28\end{array}$ & $\begin{array}{l}631.95 \\
623.32 \\
627.88 \\
624.58 \\
654.95\end{array}$ & $\begin{array}{l}401.53 \\
401.39 \\
399.77 \\
402.01 \\
401.67\end{array}$ \\
\hline $\begin{array}{l}299-E 33-42 \\
299-E 33-43 \\
299-E 33-5 \\
299-E 33-7 \\
299-E 33-8\end{array}$ & $\begin{array}{l}N \\
N \\
N \\
N\end{array}$ & $\begin{array}{l}252.58 \\
261.08 \\
232.96 \\
226.12 \\
249.20\end{array}$ & $\begin{array}{l}654.30 \\
662.68 \\
634.72 \\
626.58 \\
650.73\end{array}$ & $\begin{array}{l}401.72 \\
401.60 \\
401.76 \\
400.46 \\
401.53\end{array}$ \\
\hline $\begin{array}{l}299-E 34-1 \\
299-E 34-10 \\
299-E 34-11 \\
299-E 34-12 \\
299-E 34-2\end{array}$ & $\begin{array}{l}N \\
N\end{array}$ & $\begin{array}{l}227.57 \\
237.96 \\
216.14 \\
237.23 \\
229.17\end{array}$ & $\begin{array}{l}629.45 \\
639.77 \\
617.93 \\
638.83 \\
630.80\end{array}$ & $\begin{array}{l}401.88 \\
401.81 \\
401.79 \\
401.60 \\
401.63\end{array}$ \\
\hline
\end{tabular}


Appendix A: December 1993 Water Level Measurement Data (Sheet 9 of 22)

\begin{tabular}{|c|c|c|c|c|}
\hline \multirow{2}{*}{ Well } & \multirow{2}{*}{ Notes } & \multirow{2}{*}{$\begin{array}{l}\text { Depth to } \\
\text { Water, ft }\end{array}$} & \multicolumn{2}{|c|}{ Elevation, $\mathrm{ft}$ above $\mathrm{ms} 1$} \\
\hline & & & Adjusted Casing & Water Level \\
\hline $\begin{array}{l}299-E 34-3 \\
299-E 34-5 \\
299-E 34-6 \\
299-E 34-7 \\
299-E 34-8\end{array}$ & & $\begin{array}{l}209.66 \\
188.01 \\
196.16 \\
202.56 \\
239.25\end{array}$ & $\begin{array}{l}611.52 \\
590.79 \\
597.83 \\
604.25 \\
640.52\end{array}$ & $\begin{array}{l}401.86 \\
402.78 \\
401.67 \\
401.69 \\
401.27\end{array}$ \\
\hline $\begin{array}{l}299-E 34-9 \\
299-E 35-1 \\
299-E 35-2 \\
299-W 10-10 \\
299-W 10-11\end{array}$ & $\mathbf{N}$ & $\begin{array}{l}226.91 \\
195.31 \\
199.95 \\
214.19 \\
214.08\end{array}$ & $\begin{array}{l}628.69 \\
598.30 \\
602.10 \\
675.09 \\
675.26\end{array}$ & $\begin{array}{l}401.78 \\
402.99 \\
402.15 \\
460.90 \\
461.18\end{array}$ \\
\hline $\begin{array}{l}299-W 10-12 \\
299-W 10-13 \\
299-W 10-14 \\
299-W 10-15 \\
299-W 10-16\end{array}$ & & $\begin{array}{l}213.79 \\
237.62 \\
238.37 \\
214.77 \\
211.48\end{array}$ & $\begin{array}{l}674.75 \\
699.04 \\
699.43 \\
675.64 \\
672.76\end{array}$ & $\begin{array}{l}460.96 \\
461.42 \\
461.06 \\
460.87 \\
461.28\end{array}$ \\
\hline $\begin{array}{l}299-W 10-17 \\
299-W 10-18 \\
299-W 10-19 \\
299-W 10-2 \\
299-W 10-5\end{array}$ & $\begin{array}{l}\mathbf{N} \\
\mathbf{N}\end{array}$ & $\begin{array}{l}209.22 \\
208.41 \\
222.91 \\
212.78 \\
210.32\end{array}$ & $\begin{array}{l}670.84 \\
670.93 \\
682.99 \\
673.99 \\
672.31\end{array}$ & $\begin{array}{l}461.62 \\
462.52 \\
460.08 \\
461.21 \\
461.99\end{array}$ \\
\hline $\begin{array}{l}299-W 10-8 \\
299-W 10-9 \\
299-W 11-10 \\
299-W 11-12 \\
299-W 11-14\end{array}$ & $\begin{array}{l}N \\
N\end{array}$ & $\begin{array}{l}219.48 \\
213.58 \\
275.85 \\
218.17 \\
257.97\end{array}$ & $\begin{array}{l}680.05 \\
674.67 \\
728.89 \\
679.26 \\
715.16\end{array}$ & $\begin{array}{l}460.57 \\
461.09 \\
453.04 \\
461.09 \\
457.19\end{array}$ \\
\hline $\begin{array}{l}299-W 11-19 \\
299-W 11-23 \\
299-W 11-24 \\
299-W 11-27 \\
299-W 11-6\end{array}$ & $N$ & $\begin{array}{l}250.39 \\
227.58 \\
226.39 \\
224.49 \\
258.52\end{array}$ & $\begin{array}{l}707.00 \\
687.88 \\
687.17 \\
685.27 \\
716.23\end{array}$ & $\begin{array}{l}456.61 \\
460.30 \\
460.78 \\
460.78 \\
457.71\end{array}$ \\
\hline $\begin{array}{l}299-W 11-7 \\
299-W 12-1 \\
299-W 14-1 \\
299-W 14-12 \\
299-W 14-9\end{array}$ & $\mathrm{~N}$ & $\begin{array}{l}249.11 \\
277.86 \\
205.86 \\
207.16 \\
226.20\end{array}$ & $\begin{array}{l}709.11 \\
726.46 \\
665.83 \\
670.52\end{array}$ & $\begin{array}{l}460.00 \\
448.60 \\
459.97 \\
463.36\end{array}$ \\
\hline
\end{tabular}


Appendix A: December 1993 Water Level Measurement Data (Sheet 10 of 22)

\begin{tabular}{|c|c|c|c|c|}
\hline \multirow{2}{*}{ Well } & \multirow{2}{*}{ Notes } & \multirow{2}{*}{$\begin{array}{l}\text { Depth to } \\
\text { Water, ft }\end{array}$} & \multicolumn{2}{|c|}{ Elevation, ft above msl } \\
\hline & & & Adjusted Casing & Water Leve1 \\
\hline $\begin{array}{l}299-W 15-10 \\
299-W 15-12 \\
299-W 15-13 \\
299-W 15-15 \\
299-W 15-16\end{array}$ & $\begin{array}{l}N \\
N \\
N\end{array}$ & $\begin{array}{l}213.63 \\
207.44 \\
207.45 \\
234.62 \\
221.11\end{array}$ & $\begin{array}{l}676.00 \\
670.07 \\
670.12 \\
697.96 \\
684.89\end{array}$ & $\begin{array}{l}462.37 \\
462.63 \\
462.67 \\
463.34 \\
463.78\end{array}$ \\
\hline $\begin{array}{l}299-W 15-17 \\
299-W 15-18 \\
299-W 15-19 \\
299-W 15-20 \\
299-W 15-22\end{array}$ & $N$ & $\begin{array}{l}220.97 \\
221.75 \\
228.39 \\
235.22 \\
206.26\end{array}$ & $\begin{array}{l}684.64 \\
685.71 \\
691.60 \\
698.36 \\
670.77\end{array}$ & $\begin{array}{l}463.67 \\
463.96 \\
463.21 \\
463.14 \\
464.51\end{array}$ \\
\hline $\begin{array}{l}299-W 15-23 \\
299-W 15-24 \\
299-W 15-4 \\
299-W 15-5 \\
299-W 18-15\end{array}$ & $N$ & $\begin{array}{l}236.24 \\
235.94 \\
198.34 \\
205.69 \\
197.59\end{array}$ & $\begin{array}{l}699.49 \\
699.37 \\
662.00 \\
670.68 \\
660.76\end{array}$ & $\begin{array}{l}463.25 \\
463.43 \\
463.66 \\
464.99 \\
463.17\end{array}$ \\
\hline $\begin{array}{l}299-W 18-21 \\
299-W 18-22 \\
299-W 18-23 \\
299-W 18-24 \\
299-W 18-25\end{array}$ & $\begin{array}{l}N \\
N\end{array}$ & $\begin{array}{l}205.29 \\
205.58 \\
233.42 \\
220.24 \\
201.87\end{array}$ & $\begin{array}{l}668.62 \\
668.49 \\
696.81 \\
684.35 \\
666.04\end{array}$ & $\begin{array}{l}463.33 \\
462.91 \\
463.39 \\
464.11 \\
464.17\end{array}$ \\
\hline $\begin{array}{l}299-W 18-26 \\
299-W 18-27 \\
299-W 18-28 \\
299-W 18-29 \\
299-W 18-30\end{array}$ & $\begin{array}{l}N \\
N \\
Q \\
N\end{array}$ & $\begin{array}{l}235.65 \\
226.79 \\
216.37 \\
126.62 \\
208.43\end{array}$ & $\begin{array}{l}699.05 \\
690.25 \\
679.99 \\
674.14 \\
672.84\end{array}$ & $\begin{array}{l}463.40 \\
463.46 \\
463.62 \\
547.52 \\
464.41\end{array}$ \\
\hline $\begin{array}{l}299-W 18-31 \\
299-W 18-32 \\
299-W 18-33 \\
299-W 19-1 \\
299-W 19-12\end{array}$ & $\begin{array}{l}N \\
N \\
N \\
P\end{array}$ & $\begin{array}{l}199.88 \\
211.93 \\
205.23 \\
208.34 \\
208.81\end{array}$ & $\begin{array}{l}664.16 \\
676.65 \\
673.77 \\
673.25\end{array}$ & $\begin{array}{l}464.28 \\
464.72 \\
465.43 \\
464.44\end{array}$ \\
\hline $\begin{array}{l}299-W 19-14 \\
299-W 19-15 \\
299-W 19-2 \\
299-W 19-20 \\
299-W 19-21\end{array}$ & $N$ & $\begin{array}{l}233.82 \\
233.12 \\
237.10 \\
235.86 \\
213.88\end{array}$ & $\begin{array}{l}693.21 \\
693.28 \\
694.04 \\
691.04 \\
678.53\end{array}$ & $\begin{array}{l}459.39 \\
460.16 \\
456.94 \\
455.18 \\
464.65\end{array}$ \\
\hline
\end{tabular}


Appendix A: December 1993 liater Level Measurement Data (Sheet 11 of 22)

\begin{tabular}{|c|c|c|c|c|}
\hline \multirow{2}{*}{ We 71} & \multirow{2}{*}{ Notes } & \multirow{2}{*}{$\begin{array}{l}\text { Depth to } \\
\text { Water, ft }\end{array}$} & \multicolumn{2}{|c|}{ Elevation, ft above msl } \\
\hline & & & Adjusted Casing & Water Leve1 \\
\hline $\begin{array}{l}299-W 19-27 \\
299-W 19-28 \\
299-W 19-29 \\
299-W 19-31 \\
299-W 19-32\end{array}$ & $\begin{array}{l}\mathrm{N} \\
\mathrm{N}\end{array}$ & $\begin{array}{l}219.42 \\
245.80 \\
245.20 \\
209.77 \\
210.33\end{array}$ & $\begin{array}{l}683.65 \\
703.35 \\
702.13 \\
674.19 \\
674.90\end{array}$ & $\begin{array}{l}464.23 \\
457.55 \\
456.93 \\
464.42 \\
464.57\end{array}$ \\
\hline $\begin{array}{l}299-W 19-4 \\
299-W 19-6 \\
299-W 19-91 \\
299-W 19-92 \\
299-W 21-1\end{array}$ & $\begin{array}{l}N \\
N \\
N\end{array}$ & $\begin{array}{r}259.42 \\
226.81 \\
99.06 \\
99.17 \\
247.31\end{array}$ & $\begin{array}{l}715.26 \\
686.60 \\
677.90 \\
677.90 \\
699.26\end{array}$ & $\begin{array}{l}455.84 \\
459.79 \\
578.84 \\
578.73 \\
451.95\end{array}$ \\
\hline $\begin{array}{l}299-W 22-19 \\
299-W 22-21 \\
299-W 22-26 \\
299-W 22-39 \\
299-W 22-40\end{array}$ & N & $\begin{array}{l}227.51 \\
214.49 \\
222.40 \\
210.35 \\
236.42\end{array}$ & $\begin{array}{l}681.26 \\
670.00 \\
680.30 \\
668.26 \\
692.23\end{array}$ & $\begin{array}{l}453.75 \\
455.51 \\
457.90 \\
457.91 \\
455.81\end{array}$ \\
\hline $\begin{array}{l}299-W 22-41 \\
299-W 22-42 \\
299-W 22-43 \\
299-W 22-45 \\
299-W 22-46\end{array}$ & $\begin{array}{l}N \\
N\end{array}$ & $\begin{array}{l}235.97 \\
235.45 \\
234.73 \\
207.58 \\
213.51\end{array}$ & $\begin{array}{l}691.74 \\
691.16 \\
691.35 \\
666.21 \\
671.18\end{array}$ & $\begin{array}{l}455.77 \\
455.71 \\
456.62 \\
458.63 \\
457.67\end{array}$ \\
\hline $\begin{array}{l}299-W 22-7 \\
299-W 23-11 \\
299-W 23-13 \\
299-W 23-14 \\
299-W 23-15\end{array}$ & $\begin{array}{l}N \\
N \\
N\end{array}$ & $\begin{array}{l}231.89 \\
203.96 \\
205.72 \\
204.16 \\
196.82\end{array}$ & $\begin{array}{l}687.41 \\
664.14 \\
666.33 \\
664.00 \\
655.44\end{array}$ & $\begin{array}{l}455.52 \\
460.18 \\
460.61 \\
459.84 \\
458.62\end{array}$ \\
\hline $\begin{array}{l}299-W 23-4 \\
299-W 23-6 \\
299-W 23-8 \\
299-W 26-10 \\
299-W 26-11\end{array}$ & $\begin{array}{l}N \\
N \\
Q\end{array}$ & $\begin{array}{l}201.67 \\
208.97 \\
203.93 \\
214.08 \\
138.95\end{array}$ & $\begin{array}{l}662.63 \\
666.76 \\
663.95 \\
670.87 \\
674.40\end{array}$ & $\begin{array}{l}460.96 \\
457.79 \\
460.02 \\
456.79 \\
535.45\end{array}$ \\
\hline $\begin{array}{l}299-W 26-12 \\
299-W 26-6 \\
299-W 26-7 \\
299-W 26-8 \\
299-W 26-9\end{array}$ & $\begin{array}{l}N \\
N\end{array}$ & $\begin{array}{l}218.62 \\
195.74 \\
193.47 \\
208.87 \\
197.01\end{array}$ & $\begin{array}{l}675.69 \\
651.99 \\
666.31 \\
654.16\end{array}$ & $\begin{array}{l}457.07 \\
458.52 \\
457.44 \\
457.15\end{array}$ \\
\hline
\end{tabular}


Appendix A: December 1993 Water Level Measurement Data (Sheet 12 of 22)

\begin{tabular}{|c|c|c|c|c|}
\hline \multirow{2}{*}{ Well } & \multirow{2}{*}{ Notes } & \multirow{2}{*}{$\begin{array}{l}\text { Depth to } \\
\text { Water, ft }\end{array}$} & \multicolumn{2}{|c|}{ Elevation, ft above msl } \\
\hline & & & Adjusted Casing & Water Level \\
\hline $\begin{array}{l}299-W 27-2 \\
299-W 6-1 \\
299-W 6-10 \\
299-W 6-11 \\
299-W 6-12\end{array}$ & & $\begin{array}{l}220.33 \\
247.51 \\
254.95 \\
248.03 \\
236.60\end{array}$ & $\begin{array}{l}702.53 \\
712.48 \\
702.86 \\
692.51\end{array}$ & $\begin{array}{l}455.02 \\
457.53 \\
454.83 \\
455.91\end{array}$ \\
\hline $\begin{array}{l}299-W 6-2 \\
299-W 6-3 \\
299-W 6-4 \\
299-W 6-6 \\
299-W 6-7\end{array}$ & $\begin{array}{l}N \\
N \\
N \\
N\end{array}$ & $\begin{array}{l}235.39 \\
242.91 \\
243.73 \\
256.03 \\
256.27\end{array}$ & $\begin{array}{l}692.45 \\
699.83 \\
701.25 \\
710.00 \\
710.28\end{array}$ & $\begin{array}{l}457.06 \\
456.92 \\
457.52 \\
453.97 \\
454.01\end{array}$ \\
\hline $\begin{array}{l}299-W 6-9 \\
299-W 7-1 \\
299-W 7-10 \\
299-W 7-11 \\
299-W 7-12\end{array}$ & $\begin{array}{l}N \\
N \\
N\end{array}$ & $\begin{array}{l}238.59 \\
233.00 \\
233.28 \\
224.01 \\
230.18\end{array}$ & $\begin{array}{l}710.00 \\
690.71 \\
689.66 \\
681.45 \\
687.93\end{array}$ & $\begin{array}{l}471.41 \\
457.71 \\
456.38 \\
457.44 \\
457.75\end{array}$ \\
\hline $\begin{array}{l}299-W 7-2 \\
299-W 7-3 \\
299-W 7-4 \\
299-W 7-5 \\
299-W 7-6\end{array}$ & $N$ & $\begin{array}{l}218.94 \\
220.54 \\
213.21 \\
216.87 \\
222.59\end{array}$ & $\begin{array}{l}675.59 \\
676.14 \\
671.69 \\
673.05 \\
678.64\end{array}$ & $\begin{array}{l}456.65 \\
455.60 \\
458.48 \\
456.18 \\
456.05\end{array}$ \\
\hline $\begin{array}{l}299-W 7-7 \\
299-W 7-8 \\
299-W 7-9 \\
299-W 8-1 \\
299-W 9-1\end{array}$ & & $\begin{array}{l}218.69 \\
232.48 \\
233.94 \\
243.19 \\
277.23\end{array}$ & $\begin{array}{l}674.94 \\
687.35 \\
692.09 \\
701.33 \\
737.73\end{array}$ & $\begin{array}{l}456.25 \\
454.87 \\
458.15 \\
458.14 \\
460.50\end{array}$ \\
\hline $\begin{array}{l}399-1-1 \\
399-1-10 A \\
399-1-11 \\
399-1-12 \\
399-1-13 A\end{array}$ & & $\begin{array}{l}34.27 \\
31.15 \\
35.41 \\
42.33 \\
46.52\end{array}$ & $\begin{array}{l}376.66 \\
373.57 \\
377.72 \\
384.41 \\
388.62\end{array}$ & $\begin{array}{l}342.39 \\
342.42 \\
342.31 \\
342.08 \\
342.10\end{array}$ \\
\hline $\begin{array}{l}399-1-14 A \\
399-1-15 \\
399-1-16 A \\
399-1-16 B \\
399-1-16 C\end{array}$ & & $\begin{array}{r}40.87 \\
37.10 \\
39.38 \\
38.90 \\
8.09\end{array}$ & $\begin{array}{l}383.18 \\
379.53 \\
381.47 \\
381.08 \\
382.23\end{array}$ & $\begin{array}{l}342.31 \\
342.43 \\
342.09 \\
342.18 \\
374.14\end{array}$ \\
\hline
\end{tabular}


Appendix A: December 1993 Water Level Measurement Data (Sheet 13 of 22)

\begin{tabular}{|c|c|c|c|c|}
\hline \multirow{2}{*}{ Well } & \multirow{2}{*}{ Notes } & \multirow{2}{*}{$\begin{array}{l}\text { Depth to } \\
\text { Water, ft }\end{array}$} & \multicolumn{2}{|c|}{ Elevation, ft above msl } \\
\hline & & & Adjusted Casing & Water Level \\
\hline $\begin{array}{l}399-1-17 A \\
399-1-17 B \\
399-1-17 C \\
399-1-18 A \\
399-1-18 B\end{array}$ & $\begin{array}{l}\mathrm{N} \\
\mathrm{N} \\
\mathrm{N}\end{array}$ & $\begin{array}{r}35.45 \\
35.72 \\
2.18 \\
47.93 \\
46.72\end{array}$ & $\begin{array}{l}377.44 \\
377.80 \\
378.06 \\
390.78 \\
389.89\end{array}$ & $\begin{array}{l}341.99 \\
342.08 \\
375.88 \\
342.85 \\
343.17\end{array}$ \\
\hline $\begin{array}{l}399-1-18 C \\
399-1-19 \\
399-1-2 \\
399-1-21 A \\
399-1-21 B\end{array}$ & $\begin{array}{l}N \\
N \\
N \\
N\end{array}$ & $\begin{array}{l}44.36 \\
32.52 \\
45.01 \\
40.34 \\
41.30\end{array}$ & $\begin{array}{l}388.01 \\
374.64 \\
384.52 \\
382.39 \\
383.33\end{array}$ & $\begin{array}{l}343.65 \\
342.12 \\
339.51 \\
342.05 \\
342.03\end{array}$ \\
\hline $\begin{array}{l}399-1-3 \\
399-1-4 \\
399-1-5 \\
399-1-6 \\
399-1-7\end{array}$ & $\begin{array}{l}N \\
N\end{array}$ & $\begin{array}{l}42.94 \\
38.27 \\
37.68 \\
31.27 \\
43.52\end{array}$ & $\begin{array}{l}384.71 \\
380.58 \\
379.77 \\
373.81 \\
385.60\end{array}$ & $\begin{array}{l}341.77 \\
342.31 \\
342.09 \\
342.54 \\
342.08\end{array}$ \\
\hline $\begin{array}{l}399-1-8 \\
399-1-9 \\
399-2-1 \\
399-2-2 \\
399-2-3\end{array}$ & $\begin{array}{l}N \\
Q\end{array}$ & $\begin{array}{l}42.81 \\
27.63 \\
33.15 \\
35.43 \\
33.34\end{array}$ & $\begin{array}{l}384.88 \\
384.76 \\
375.23 \\
377.52 \\
375.42\end{array}$ & $\begin{array}{l}342.07 \\
357.13 \\
342.08 \\
342.09 \\
342.08\end{array}$ \\
\hline $\begin{array}{l}399-3-1 \\
399-3-10 \\
399-3-12 \\
399-3-6 \\
399-3-9\end{array}$ & $N$ & $\begin{array}{l}42.39 \\
43.39 \\
46.34 \\
50.97 \\
46.25\end{array}$ & $\begin{array}{l}384.39 \\
385.35 \\
388.04 \\
392.80 \\
388.08\end{array}$ & $\begin{array}{l}342.00 \\
341.96 \\
341.70 \\
341.83 \\
341.83\end{array}$ \\
\hline $\begin{array}{l}399-4-1 \\
399-4-10 \\
399-4-11 \\
399-4-7 \\
399-4-9\end{array}$ & $N$ & $\begin{array}{l}53.79 \\
36.56 \\
62.61 \\
36.64 \\
40.16\end{array}$ & $\begin{array}{l}395.60 \\
378.54 \\
404.45 \\
378.56 \\
382.16\end{array}$ & $\begin{array}{l}341.81 \\
341.98 \\
341.84 \\
341.92 \\
342.00\end{array}$ \\
\hline $\begin{array}{l}399-5-1 \\
399-6-1 \\
399-8-1 \\
399-8-2 \\
399-8-3\end{array}$ & $N$ & $\begin{array}{l}53.63 \\
46.81 \\
54.14 \\
55.61 \\
52.58\end{array}$ & $\begin{array}{l}395.53 \\
388.79 \\
396.13 \\
398.02 \\
394.75\end{array}$ & $\begin{array}{l}341.90 \\
341.98 \\
341.99 \\
342.41 \\
342.17\end{array}$ \\
\hline
\end{tabular}


Appendix A: December 1993 Water Level Measurement Data

(Sheet 14 of 22)

\begin{tabular}{|c|c|c|c|c|}
\hline \multirow{2}{*}{ Well } & \multirow{2}{*}{ Notes } & \multirow{2}{*}{$\begin{array}{l}\text { Depth to } \\
\text { Water, ft }\end{array}$} & \multicolumn{2}{|c|}{ Elevation, $\mathrm{ft}$ above $\mathrm{msl}$} \\
\hline & & & Adjusted Casing & Water Level \\
\hline $\begin{array}{l}399-8-5 A \\
399-8-5 B \\
399-8-5 C \\
3099-47-18 B \\
699-101-48 B\end{array}$ & $N$ & $\begin{array}{l}57.95 \\
56.91 \\
32.29 \\
33.57 \\
12.58\end{array}$ & $\begin{array}{l}400.31 \\
399.85 \\
399.91 \\
390.15\end{array}$ & $\begin{array}{l}342.36 \\
342.94 \\
367.62 \\
377.57\end{array}$ \\
\hline $\begin{array}{l}699-20-20 \\
699-20-39 \\
699-20-41 P \\
699-20-41 Q \\
699-20-41 R\end{array}$ & $\begin{array}{l}P \\
P \\
P\end{array}$ & $\begin{array}{l}105.92 \\
138.58 \\
134.13 \\
135.16 \\
137.41\end{array}$ & $\begin{array}{l}505.58 \\
539.98\end{array}$ & $\begin{array}{l}399.66 \\
401.40\end{array}$ \\
\hline $\begin{array}{l}699-23-34 \\
699-24-33 \\
699-24-34 A \\
699-24-34 B \\
699-24-34 C\end{array}$ & & $\begin{array}{l}131.75 \\
123.20 \\
132.77 \\
132.36 \\
131.47\end{array}$ & $\begin{array}{l}532.86 \\
524.21 \\
533.89 \\
533.50 \\
532.58\end{array}$ & $\begin{array}{l}401.11 \\
401.01 \\
401.12 \\
401.14 \\
401.11\end{array}$ \\
\hline $\begin{array}{l}699-24-35 \\
699-25-33 A \\
699-25-34 A \\
699-25-34 B \\
699-25-34 C\end{array}$ & & $\begin{array}{l}137.66 \\
128.00 \\
129.23 \\
129.17 \\
134.35\end{array}$ & $\begin{array}{l}538.81 \\
528.97 \\
530.31 \\
529.40 \\
535.46\end{array}$ & $\begin{array}{l}401.15 \\
400.97 \\
401.08 \\
400.23 \\
401.11\end{array}$ \\
\hline $\begin{array}{l}699-25-34 D \\
699-25-55 \\
699-25-70 \\
699-26-33 \\
699-26-34\end{array}$ & & $\begin{array}{l}136.78 \\
264.59 \\
183.92 \\
134.63 \\
127.24\end{array}$ & $\begin{array}{l}537.91 \\
676.55 \\
629.78 \\
535.49 \\
528.40\end{array}$ & $\begin{array}{l}401.13 \\
411.96 \\
445.86 \\
400.86 \\
401.16\end{array}$ \\
\hline $\begin{array}{l}699-26-34 B \\
699-26-35 A \\
699-26-35 C \\
699-26-83 B P \\
699-26-83 B Q\end{array}$ & $\begin{array}{l}N \\
P \\
P\end{array}$ & $\begin{array}{l}129.13 \\
131.55 \\
131.51 \\
233.49 \\
233.13\end{array}$ & $\begin{array}{l}530.27 \\
532.66 \\
532.68\end{array}$ & $\begin{array}{l}401.14 \\
401.11 \\
401.17\end{array}$ \\
\hline $\begin{array}{l}699-26-83 B R \\
699-28-40 \\
699-28-52 A \\
699-29-70 A P \\
699-29-70 A Q\end{array}$ & $P$ & $\begin{array}{l}237.32 \\
158.05 \\
281.69 \\
191.11 \\
184.39\end{array}$ & $\begin{array}{l}559.44 \\
684.67 \\
629.75 \\
630.15\end{array}$ & $\begin{array}{l}401.39 \\
402.98 \\
438.64 \\
445.76\end{array}$ \\
\hline
\end{tabular}


Appendix A: December 1993 Water Level Measurement Data (Sheet 15 of 22)

\begin{tabular}{|c|c|c|c|c|}
\hline \multirow{2}{*}{ Well } & \multirow{2}{*}{ Notes } & \multirow{2}{*}{$\begin{array}{l}\text { Depth to } \\
\text { Water, ft }\end{array}$} & \multicolumn{2}{|c|}{ Elevation, ft above msl } \\
\hline & & & Adjusted Casing & Water Level \\
\hline $\begin{array}{l}699-29-70 C P \\
699-29-70 C Q \\
699-29-70 C R \\
699-29-70 C S \\
699-29-70 C T\end{array}$ & $\begin{array}{l}P \\
P \\
P \\
P \\
P\end{array}$ & $\begin{array}{l}229.96 \\
232.76 \\
231.07 \\
233.73 \\
234.65\end{array}$ & $\begin{array}{l}630.94 \\
630.99 \\
631.15 \\
631.31 \\
631.48\end{array}$ & $\begin{array}{l}400.98 \\
398.23 \\
400.08 \\
397.58 \\
396.83\end{array}$ \\
\hline $\begin{array}{l}699-29-70 C U \\
699-29-70 D P \\
699-29-78 \\
699-31-31 \\
699-31-65\end{array}$ & $\begin{array}{l}P \\
P\end{array}$ & $\begin{array}{l}235.78 \\
208.56 \\
187.75 \\
128.27 \\
243.99\end{array}$ & $\begin{array}{l}631.64 \\
632.42 \\
647.05 \\
529.32 \\
683.09\end{array}$ & $\begin{array}{l}395.86 \\
423.86 \\
459.30 \\
401.05 \\
439.10\end{array}$ \\
\hline $\begin{array}{l}699-31-84 B \\
699-32-43 \\
699-32-62 \\
699-32-70 B \\
699-32-72\end{array}$ & $N$ & $\begin{array}{l}204.47 \\
114.92 \\
278.82 \\
217.88 \\
217.50\end{array}$ & $\begin{array}{l}625.12 \\
516.62 \\
707.09 \\
666.68 \\
668.16\end{array}$ & $\begin{array}{l}420.65 \\
401.70 \\
428.27 \\
448.80 \\
450.66\end{array}$ \\
\hline $\begin{array}{l}699-32-77 \\
699-33-42 \\
699-33-56 \\
699-34-39 A \\
699-34-41 B\end{array}$ & & $\begin{array}{l}195.81 \\
114.98 \\
314.37 \\
135.60 \\
169.10\end{array}$ & $\begin{array}{l}653.74 \\
516.00 \\
717.03 \\
537.07 \\
570.89\end{array}$ & $\begin{array}{l}457.93 \\
401.02 \\
402.66 \\
401.47 \\
401.79\end{array}$ \\
\hline $\begin{array}{l}699-34-42 \\
699-34-51 \\
699-35-66 \\
699-35-70 \\
699-35-78 A\end{array}$ & & $\begin{array}{l}138.32 \\
334.79 \\
287.76 \\
244.94 \\
198.03\end{array}$ & $\begin{array}{l}540.20 \\
736.76 \\
725.65 \\
693.72 \\
660.65\end{array}$ & $\begin{array}{l}401.88 \\
401.97 \\
437.89 \\
448.78 \\
462.62\end{array}$ \\
\hline $\begin{array}{l}699-36-61 A \\
699-37-43 \\
699-37-82 A \\
699-38-65 \\
699-38-70\end{array}$ & & $\begin{array}{l}341.61 \\
287.19 \\
172.22 \\
324.41 \\
260.61\end{array}$ & $\begin{array}{l}748.11 \\
690.17 \\
636.75 \\
753.33 \\
710.67\end{array}$ & $\begin{array}{l}406.50 \\
402.98 \\
464.53 \\
428.92 \\
450.06\end{array}$ \\
\hline $\begin{array}{l}699-39-39 \\
699-39-79 \\
699-40-33 A \\
699-40-36 \\
699-40-39\end{array}$ & & $\begin{array}{l}125.17 \\
210.29 \\
107.63 \\
118.12 \\
129.71\end{array}$ & $\begin{array}{l}536.65 \\
673.52 \\
518.05 \\
528.92 \\
541.84\end{array}$ & $\begin{array}{l}411.48 \\
463.23 \\
410.42 \\
410.80 \\
412.13\end{array}$ \\
\hline
\end{tabular}


Appendix A: December 1993 Water Level Measurement Data (Sheet 16 of 22)

\begin{tabular}{|c|c|c|c|c|}
\hline \multirow{2}{*}{ Well } & \multirow{2}{*}{ Notes } & \multirow{2}{*}{$\begin{array}{l}\text { Depth to } \\
\text { Water, ft }\end{array}$} & \multicolumn{2}{|c|}{ Elevation, ft above msl } \\
\hline & & & Adjusted Casing & Water Level \\
\hline $\begin{array}{l}699-40-40 A \\
699-40-40 B \\
699-40-62 \\
699-41-35 \\
699-41-40\end{array}$ & $\begin{array}{l}N \\
N\end{array}$ & $\begin{array}{l}130.16 \\
130.81 \\
343.17 \\
108.63 \\
131.00\end{array}$ & $\begin{array}{l}541.21 \\
542.18 \\
747.78 \\
520.38 \\
545.94\end{array}$ & $\begin{array}{l}411.05 \\
411.37 \\
404.61 \\
411.75 \\
414.94\end{array}$ \\
\hline $\begin{array}{l}699-41-42 \\
699-42-37 \\
699-42-39 A \\
699-42-39 B \\
699-42-40 A\end{array}$ & $\begin{array}{l}N \\
N\end{array}$ & $\begin{array}{l}232.72 \\
105.09 \\
139.77 \\
140.27 \\
123.77\end{array}$ & $\begin{array}{l}643.91 \\
519.42 \\
558.14 \\
558.32 \\
545.53\end{array}$ & $\begin{array}{l}411.19 \\
414.33 \\
418.37 \\
418.05 \\
421.76\end{array}$ \\
\hline $\begin{array}{l}699-42-40 B \\
699-42-40 C \\
699-42-41 \\
699-42-42 B \\
699-43-40\end{array}$ & $\begin{array}{l}P \\
N \\
N\end{array}$ & $\begin{array}{l}124.56 \\
133.84 \\
146.54 \\
166.05 \\
123.73\end{array}$ & $\begin{array}{l}546.46 \\
546.16 \\
567.30 \\
583.23 \\
542.20\end{array}$ & $\begin{array}{l}421.90 \\
412.32 \\
420.76 \\
417.18 \\
418.47\end{array}$ \\
\hline $\begin{array}{l}699-43-42 J \\
699-43-43 \\
699-43-45 \\
699-43-91 A P \\
699-43-91 A Q\end{array}$ & $\begin{array}{l}P \\
P\end{array}$ & $\begin{array}{l}162.87 \\
164.40 \\
194.78 \\
231.68 \\
231.71\end{array}$ & $\begin{array}{l}581.68 \\
579.37 \\
597.68 \\
671.51 \\
671.94\end{array}$ & $\begin{array}{l}418.81 \\
414.97 \\
402.90 \\
439.83 \\
440.23\end{array}$ \\
\hline $\begin{array}{l}699-43-91 D \\
699-44-39 B \\
699-44-42 \\
699-44-43 B \\
699-44-64\end{array}$ & $P$ & $\begin{array}{r}262.11 \\
95.34 \\
158.55 \\
164.77 \\
319.76\end{array}$ & $\begin{array}{l}671.99 \\
513.40 \\
579.22 \\
580.12 \\
725.60\end{array}$ & $\begin{array}{l}409.88 \\
418.06 \\
420.67 \\
415.35 \\
405.84\end{array}$ \\
\hline $\begin{array}{l}699-44-91 P \\
699-44-91 Q \\
699-44-91 R \\
699-44-91 S \\
699-44-91 T\end{array}$ & $\begin{array}{l}p \\
p \\
p \\
p \\
P\end{array}$ & $\begin{array}{l}268.26 \\
268.52 \\
267.33 \\
274.50 \\
275.73\end{array}$ & $\begin{array}{l}672.15 \\
672.33 \\
672.49 \\
672.68 \\
672.84\end{array}$ & $\begin{array}{l}403.89 \\
403.81 \\
405.16 \\
398.18 \\
397.11\end{array}$ \\
\hline $\begin{array}{l}699-44-91 U \\
699-45-42 \\
699-45-69 \\
699-47-35 A \\
699-47-35 B\end{array}$ & $\begin{array}{l}P \\
Q\end{array}$ & $\begin{array}{r}276.02 \\
161.23 \\
279.69 \\
63.20 \\
63.47\end{array}$ & $\begin{array}{l}673.01 \\
577.33 \\
476.36 \\
476.65\end{array}$ & $\begin{array}{l}396.99 \\
416.10 \\
413.16 \\
413.18\end{array}$ \\
\hline
\end{tabular}


Appendix A: December 1993 Water Level Measurement Data (Sheet 17 of 22)

\begin{tabular}{|c|c|c|c|c|}
\hline \multirow{2}{*}{ Well } & \multirow{2}{*}{ Notes } & \multirow{2}{*}{$\begin{array}{l}\text { Depth to } \\
\text { Water, ft }\end{array}$} & \multicolumn{2}{|c|}{ Elevation, $\mathrm{ft}$ above $\mathrm{ms}$} \\
\hline & & & Adjusted Casing & Water Level \\
\hline $\begin{array}{l}699-47-46 A \\
699-47-50 \\
699-47-60 \\
699-47-80 A P \\
699-47-80 A Q\end{array}$ & $\begin{array}{l}p \\
p\end{array}$ & $\begin{array}{l}176.99 \\
181.13 \\
249.96 \\
272.42 \\
262.78\end{array}$ & $\begin{array}{l}580.14 \\
584.22 \\
651.52 \\
713.03 \\
713.51\end{array}$ & $\begin{array}{l}403.15 \\
403.09 \\
401.56 \\
440.61 \\
450.73\end{array}$ \\
\hline $\begin{array}{l}699-47-80 C P \\
699-47-80 C Q \\
699-47-80 C R \\
699-47-80 C S \\
699-47-80 C T\end{array}$ & $\begin{array}{l}p \\
p \\
p \\
p \\
P\end{array}$ & $\begin{array}{l}307.99 \\
309.02 \\
308.97 \\
314.89 \\
315.30\end{array}$ & $\begin{array}{l}712.58 \\
712.75 \\
712.92 \\
713.10 \\
713.25\end{array}$ & $\begin{array}{l}404.59 \\
403.73 \\
403.95 \\
398.21 \\
397.95\end{array}$ \\
\hline $\begin{array}{l}699-47-80 C U \\
699-47-80 D P \\
699-48-50 \\
699-48-71 \\
699-49-55 A\end{array}$ & $\begin{array}{l}P \\
P \\
N\end{array}$ & $\begin{array}{l}315.45 \\
296.34 \\
170.88 \\
243.96 \\
129.37\end{array}$ & $\begin{array}{l}713.43 \\
574.06 \\
688.15 \\
531.03\end{array}$ & $\begin{array}{l}397.98 \\
403.18 \\
444.19 \\
401.66\end{array}$ \\
\hline $\begin{array}{l}699-49-55 B \\
699-49-57 A \\
699-49-57 B \\
699-49-79 \\
699-50-42\end{array}$ & $\begin{array}{l}P \\
N \\
N\end{array}$ & $\begin{array}{r}129.39 \\
152.04 \\
154.41 \\
234.00 \\
56.66\end{array}$ & $\begin{array}{l}531.12 \\
553.52 \\
555.99 \\
689.20 \\
466.84\end{array}$ & $\begin{array}{l}401.73 \\
401.48 \\
401.58 \\
455.20 \\
410.18\end{array}$ \\
\hline $\begin{array}{l}699-50-45 \\
699-50-48 B \\
699-50-53 A \\
699-50-53 B \\
699-50-85\end{array}$ & $\begin{array}{l}N \\
N\end{array}$ & $\begin{array}{r}43.73 \\
145.36 \\
155.89 \\
155.83 \\
284.66\end{array}$ & $\begin{array}{l}451.41 \\
550.39 \\
557.46 \\
557.62 \\
739.35\end{array}$ & $\begin{array}{l}407.68 \\
405.03 \\
401.57 \\
401.79 \\
454.69\end{array}$ \\
\hline $\begin{array}{l}699-51-46 \\
699-51-63 \\
699-51-75 \\
699-52-46 A \\
699-52-48\end{array}$ & $\begin{array}{l}P \\
P\end{array}$ & $\begin{array}{r}38.22 \\
167.83 \\
193.11 \\
47.78 \\
62.13\end{array}$ & $\begin{array}{l}444.63 \\
571.84 \\
641.51 \\
455.61 \\
466.06\end{array}$ & $\begin{array}{l}406.41 \\
404.01 \\
448.40 \\
407.83 \\
403.93\end{array}$ \\
\hline $\begin{array}{l}699-52-54 \\
699-52-57 \\
699-53-35 \\
699-53-47 A \\
699-53-47 B\end{array}$ & $\begin{array}{l}N \\
N\end{array}$ & $\begin{array}{r}167.11 \\
160.71 \\
130.56 \\
32.85 \\
33.17\end{array}$ & $\begin{array}{l}568.45 \\
561.80 \\
530.99 \\
438.28 \\
438.58\end{array}$ & $\begin{array}{l}401.34 \\
401.09 \\
400.43 \\
405.43 \\
405.41\end{array}$ \\
\hline
\end{tabular}


Appendix A: December 1993 Water Level Measurement Data (Sheet 18 of 22)

\begin{tabular}{|c|c|c|c|c|}
\hline \multirow{2}{*}{ Well } & \multirow{2}{*}{ Notes } & \multirow{2}{*}{$\begin{array}{l}\text { Depth to } \\
\text { Water, ft }\end{array}$} & \multicolumn{2}{|c|}{ Elevation, ft above $\mathrm{msl}$} \\
\hline & & & Adjusted Casing & Water Level \\
\hline $\begin{array}{l}699-53-48 A \\
699-53-48 B \\
699-53-50 \\
699-53-55 A \\
699-53-55 B\end{array}$ & $\begin{array}{l}p \\
p\end{array}$ & $\begin{array}{r}39.40 \\
38.31 \\
40.85 \\
175.34 \\
176.06\end{array}$ & $\begin{array}{l}442.45 \\
442.71 \\
444.21 \\
576.56 \\
576.84\end{array}$ & $\begin{array}{l}403.05 \\
404.40 \\
403.36 \\
401.22 \\
400.78\end{array}$ \\
\hline $\begin{array}{l}699-53-55 C \\
699-54-42 \\
699-54-48 \\
699-54-57 \\
699-55-40\end{array}$ & $P$ & $\begin{array}{r}175.07 \\
115.30 \\
56.06 \\
175.04 \\
134.46\end{array}$ & $\begin{array}{l}576.13 \\
511.49 \\
457.02 \\
576.24 \\
543.13\end{array}$ & $\begin{array}{l}401.06 \\
396.19 \\
400.96 \\
401.20 \\
408.67\end{array}$ \\
\hline $\begin{array}{l}699-55-50 C \\
699-55-70 \\
699-55-76 \\
699-56-43 \\
699-56-53\end{array}$ & $\begin{array}{l}P \\
P\end{array}$ & $\begin{array}{r}43.48 \\
137.56 \\
139.74 \\
132.55 \\
32.98\end{array}$ & $\begin{array}{l}444.43 \\
569.03 \\
583.24 \\
540.42 \\
434.34\end{array}$ & $\begin{array}{l}400.95 \\
431.47 \\
443.50 \\
407.87 \\
401.36\end{array}$ \\
\hline $\begin{array}{l}699-57-25 A \\
699-57-29 A \\
699-57-29 B \\
699-57-59 \\
699-57-83 A\end{array}$ & $\begin{array}{l}N \\
N \\
N\end{array}$ & $\begin{array}{r}51.22 \\
54.33 \\
55.05 \\
175.28 \\
145.51\end{array}$ & $\begin{array}{l}414.57 \\
408.47 \\
416.18 \\
576.26 \\
577.96\end{array}$ & $\begin{array}{l}363.35 \\
354.14 \\
361.13 \\
400.98 \\
432.45\end{array}$ \\
\hline $\begin{array}{l}699-57-83 B P \\
699-57-83 B Q \\
699-57-83 B R \\
699-57-83 C \\
699-58-24\end{array}$ & $\begin{array}{l}Q \\
Q \\
Q \\
Q\end{array}$ & $\begin{array}{r}179.19 \\
179.59 \\
179.61 \\
173.78 \\
57.78\end{array}$ & $\begin{array}{l}578.56 \\
578.73 \\
578.89 \\
579.60 \\
418.80\end{array}$ & $\begin{array}{l}399.37 \\
399.14 \\
399.28 \\
405.82 \\
361.02\end{array}$ \\
\hline $\begin{array}{l}699-59-32 \\
699-59-58 \\
699-59-80 B \\
699-60-32 \\
699-60-57\end{array}$ & $N$ & $\begin{array}{r}62.95 \\
97.01 \\
153.70 \\
64.11 \\
68.53\end{array}$ & $\begin{array}{l}424.29 \\
497.77 \\
583.25 \\
425.30 \\
469.64\end{array}$ & $\begin{array}{l}361.34 \\
400.76 \\
429.55 \\
361.19 \\
401.11\end{array}$ \\
\hline $\begin{array}{l}699-60-59 \\
699-60-60 \\
699-61-37 \\
699-61-41 \\
699-61-62\end{array}$ & $\begin{array}{l}Q \\
N \\
N\end{array}$ & $\begin{array}{r}105.29 \\
111.32 \\
61.73 \\
33.45 \\
96.71\end{array}$ & $\begin{array}{l}512.03 \\
442.94 \\
428.92 \\
497.51\end{array}$ & $\begin{array}{l}400.71 \\
381.21 \\
395.47 \\
400.80\end{array}$ \\
\hline
\end{tabular}


Appendix A: December 1993 Water Level Measurement Data (Sheet 19 of 22)

\begin{tabular}{|c|c|c|c|c|}
\hline \multirow{2}{*}{ Well } & \multirow{2}{*}{ Notes } & \multirow{2}{*}{$\begin{array}{l}\text { Depth to } \\
\text { Water, ft }\end{array}$} & \multicolumn{2}{|c|}{ Elevation, $\mathrm{ft}$ above $\mathrm{msl}$} \\
\hline & & & Adjusted Casing & Water Level \\
\hline $\begin{array}{l}699-61-66 \\
699-62-31 \\
699-62-43 A \\
699-63-25 A \\
699-63-51\end{array}$ & & $\begin{array}{r}122.19 \\
72.98 \\
36.57 \\
34.20 \\
25.81\end{array}$ & $\begin{array}{l}522.18 \\
434.12 \\
432.30 \\
395.15 \\
424.54\end{array}$ & $\begin{array}{l}399.99 \\
361.14 \\
395.73 \\
360.95 \\
398.73\end{array}$ \\
\hline $\begin{array}{l}699-63-55 \\
699-63-58 \\
699-63-90 \\
699-63-92 \\
699-64-27\end{array}$ & $\begin{array}{l}\mathbf{N} \\
\mathbf{N}\end{array}$ & $\begin{array}{r}27.20 \\
92.23 \\
113.46 \\
100.54 \\
53.16\end{array}$ & $\begin{array}{l}426.54 \\
491.90 \\
509.73 \\
497.50 \\
414.29\end{array}$ & $\begin{array}{l}399.34 \\
399.67 \\
396.27 \\
396.96 \\
361.13\end{array}$ \\
\hline $\begin{array}{l}699-64-62 \\
699-65-22 \\
699-65-50 \\
699-65-59 A \\
699-65-72\end{array}$ & & $\begin{array}{r}100.84 \\
31.32 \\
68.36 \\
107.56 \\
142.87\end{array}$ & $\begin{array}{l}500.25 \\
391.10 \\
467.06 \\
506.96 \\
540.28\end{array}$ & $\begin{array}{l}399.41 \\
359.78 \\
398.70 \\
399.40 \\
397.41\end{array}$ \\
\hline $\begin{array}{l}699-65-83 \\
699-65-95 \\
699-66-103 \\
699-66-23 \\
699-66-38\end{array}$ & $\begin{array}{l}P \\
N\end{array}$ & $\begin{array}{l}88.98 \\
55.63 \\
66.74 \\
28.98 \\
34.03\end{array}$ & $\begin{array}{l}485.63 \\
452.26 \\
463.01 \\
389.01 \\
436.24\end{array}$ & $\begin{array}{l}396.65 \\
396.63 \\
396.27 \\
360.03 \\
402.21\end{array}$ \\
\hline $\begin{array}{l}699-66-39 \\
699-66-58 \\
699-66-64 \\
699-66-91 \\
699-67-51\end{array}$ & $N$ & $\begin{array}{r}49.89 \\
105.05 \\
107.19 \\
70.40 \\
125.83\end{array}$ & $\begin{array}{l}453.78 \\
503.33 \\
505.92 \\
467.75 \\
524.59\end{array}$ & $\begin{array}{l}403.89 \\
398.28 \\
398.73 \\
397.35 \\
398.76\end{array}$ \\
\hline $\begin{array}{l}699-67-86 \\
699-67-98 \\
699-68-105 \\
699-69-38 \\
699-69-450\end{array}$ & & $\begin{array}{l}76.02 \\
57.65 \\
57.44 \\
20.93 \\
89.17\end{array}$ & $\begin{array}{l}472.39 \\
455.47 \\
451.85 \\
424.10 \\
487.18\end{array}$ & $\begin{array}{l}396.37 \\
397.82 \\
394.41 \\
403.17 \\
398.01\end{array}$ \\
\hline $\begin{array}{l}699-70-23 \\
699-70-68 \\
699-71-30 \\
699-71-52 \\
699-71-77\end{array}$ & & $\begin{array}{r}30.02 \\
128.54 \\
30.53 \\
124.93 \\
77.03\end{array}$ & $\begin{array}{l}391.71 \\
526.21 \\
400.68 \\
523.04 \\
472.28\end{array}$ & $\begin{array}{l}361.69 \\
397.67 \\
370.15 \\
398.11 \\
395.25\end{array}$ \\
\hline
\end{tabular}


Appendix A: December 1993 Water Level Measurement Data (Sheet 20 of 22)

\begin{tabular}{|c|c|c|c|c|}
\hline \multirow{2}{*}{ Wel1 } & \multirow{2}{*}{ Notes } & \multirow{2}{*}{$\begin{array}{l}\text { Depth to } \\
\text { Water, ft }\end{array}$} & \multicolumn{2}{|c|}{ Elevation, ft above msl } \\
\hline & & & Adjusted Casing & Water Level \\
\hline $\begin{array}{l}699-72-73 \\
699-72-88 \\
699-72-92 \\
699-73-61 \\
699-74-44\end{array}$ & & $\begin{array}{r}87.12 \\
38.70 \\
54.44 \\
133.67 \\
48.85\end{array}$ & $\begin{array}{l}482.57 \\
437.37 \\
452.22 \\
531.53 \\
445.18\end{array}$ & $\begin{array}{l}395.45 \\
398.67 \\
397.78 \\
397.86 \\
396.33\end{array}$ \\
\hline $\begin{array}{l}699-74-48 \\
699-77-36 \\
699-77-54 \\
699-78-62 \\
699-80-435\end{array}$ & & $\begin{array}{l}90.13 \\
36.56 \\
84.33 \\
75.78 \\
25.32\end{array}$ & $\begin{array}{l}487.18 \\
412.28 \\
480.59 \\
469.88 \\
412.52\end{array}$ & $\begin{array}{l}397.05 \\
375.72 \\
396.26 \\
394.10 \\
387.20\end{array}$ \\
\hline $\begin{array}{l}699-81-38 \\
699-81-58 \\
699-82-45 A \\
699-83-36 \\
699-83-47\end{array}$ & $\mathbf{N}$ & $\begin{array}{l}27.49 \\
46.93 \\
25.15 \\
41.17 \\
46.94\end{array}$ & $\begin{array}{l}406.47 \\
439.55 \\
413.73 \\
418.63 \\
435.27\end{array}$ & $\begin{array}{l}378.98 \\
392.62 \\
388.58 \\
377.46 \\
388.33\end{array}$ \\
\hline $\begin{array}{l}699-84-35 A \\
699-86-42 \\
699-87-42 A \\
699-87-55 \\
699-88-41\end{array}$ & $P$ & $\begin{array}{r}7.18 \\
25.13 \\
32.84 \\
73.05 \\
34.03\end{array}$ & $\begin{array}{l}400.05 \\
409.92 \\
416.53 \\
458.63 \\
416.04\end{array}$ & $\begin{array}{l}392.87 \\
384.79 \\
383.69 \\
385.58 \\
382.01\end{array}$ \\
\hline $\begin{array}{l}699-89-35 \\
699-90-45 \\
699-91-37 \\
699-91-46 \\
699-92-49\end{array}$ & $N$ & $\begin{array}{l}27.36 \\
37.74 \\
50.55 \\
33.36 \\
48.95\end{array}$ & $\begin{array}{l}397.46 \\
421.54 \\
422.93 \\
417.06 \\
431.88\end{array}$ & $\begin{array}{l}370.10 \\
383.80 \\
372.38 \\
383.70 \\
382.93\end{array}$ \\
\hline $\begin{array}{l}699-93-48 \\
699-96-43 \\
699-96-49 \\
699-97-43 \\
699-97-51 A\end{array}$ & $\begin{array}{l}N \\
N\end{array}$ & $\begin{array}{l}55.33 \\
42.89 \\
38.13 \\
43.44 \\
21.57\end{array}$ & $\begin{array}{l}437.79 \\
421.84 \\
419.23 \\
421.84 \\
402.27\end{array}$ & $\begin{array}{l}382.46 \\
378.95 \\
381.10 \\
378.40 \\
380.70\end{array}$ \\
\hline $\begin{array}{l}699-98-49 A \\
699-S 18-E 2 A \\
699-S 19-E 13 \\
699-S 19-E 14 \\
699-S 22-E 9 A\end{array}$ & $N$ & $\begin{array}{l}21.31 \\
75.49 \\
51.12 \\
30.57 \\
29.86\end{array}$ & $\begin{array}{l}401.80 \\
434.85 \\
394.51 \\
373.86 \\
374.19\end{array}$ & $\begin{array}{l}380.49 \\
359.36 \\
343.39 \\
343.29 \\
344.33\end{array}$ \\
\hline
\end{tabular}


Appendix A: December 1993 Water Level Measurement Data (Sheet 21 of 22)

\begin{tabular}{|c|c|c|c|c|}
\hline \multirow{2}{*}{ Well } & \multirow{2}{*}{ Notes } & \multirow{2}{*}{$\begin{array}{l}\text { Depth to } \\
\text { Water, ft }\end{array}$} & \multicolumn{2}{|c|}{ Elevation, ft above msl } \\
\hline & & & Adjusted Casing & Water Level \\
\hline $\begin{array}{l}699-S 22-E 9 B \\
699-S 27-E 14 \\
699-S 27-E 9 A \\
699-S 27-E 9 B \\
699-S 27-E 9 C\end{array}$ & & $\begin{array}{r}28.69 \\
57.92 \\
42.16 \\
41.03 \\
6.58\end{array}$ & $\begin{array}{l}373.71 \\
399.68 \\
390.26 \\
390.42 \\
390.56\end{array}$ & $\begin{array}{l}345.02 \\
341.76 \\
348.10 \\
349.39 \\
383.98\end{array}$ \\
\hline $\begin{array}{l}699-S 28-E 12 \\
699-S 29-E 12 \\
699-S 29-E 16 A \\
699-S 29-E 16 B \\
699-S 29-E 16 C\end{array}$ & & $\begin{array}{r}44.96 \\
41.26 \\
37.66 \\
37.80 \\
3.69\end{array}$ & $\begin{array}{l}389.76 \\
387.96 \\
379.73 \\
379.88 \\
379.48\end{array}$ & $\begin{array}{l}344.80 \\
346.70 \\
342.07 \\
342.08 \\
375.79\end{array}$ \\
\hline $\begin{array}{l}\text { 699-S30-E1OA } \\
699-S 30-E 10 B \\
699-S 30-E 15 A \\
699-S 31-1 \\
699-S 31-E 10 A\end{array}$ & & $\begin{array}{l}41.50 \\
40.87 \\
59.31 \\
82.54 \\
33.71\end{array}$ & $\begin{array}{l}392.29 \\
392.07 \\
400.14 \\
460.00 \\
384.57\end{array}$ & $\begin{array}{l}350.79 \\
351.20 \\
340.83 \\
377.46 \\
350.86\end{array}$ \\
\hline $\begin{array}{l}\text { 699-S31-E1OB } \\
699-S 31-E 10 C \\
699-S 31-E 10 D \\
699-S 31-E 8 \\
699-S 32-E 13 A\end{array}$ & & $\begin{array}{l}32.37 \\
31.62 \\
29.32 \\
19.18 \\
43.55\end{array}$ & $\begin{array}{l}383.71 \\
382.91 \\
380.58 \\
374.75 \\
390.46\end{array}$ & $\begin{array}{l}351.34 \\
351.29 \\
351.26 \\
355.57 \\
346.91\end{array}$ \\
\hline $\begin{array}{l}\text { 699-S32-E13B } \\
699-\text { S32-E8 } \\
699-S 34-E 10 \\
699-S 34-E 15 \\
699-S 36-E 12 B\end{array}$ & & $\begin{array}{l}46.54 \\
14.25 \\
26.09 \\
53.45 \\
41.82\end{array}$ & $\begin{array}{l}394.72 \\
375.50 \\
382.37 \\
408.04 \\
399.04\end{array}$ & $\begin{array}{l}348.18 \\
361.25 \\
356.28 \\
354.59 \\
357.22\end{array}$ \\
\hline $\begin{array}{l}\text { 699-S36-E13A } \\
699-S 37-E 11 \\
699-S 37-E 14 \\
699-S 38-E 11 \\
699-S 38-E 12 A\end{array}$ & & $\begin{array}{l}42.56 \\
41.49 \\
50.92 \\
40.33 \\
46.78\end{array}$ & $\begin{array}{l}399.30 \\
399.30 \\
408.28 \\
398.60 \\
404.95\end{array}$ & $\begin{array}{l}356.74 \\
357.81 \\
357.36 \\
358.27 \\
358.17\end{array}$ \\
\hline $\begin{array}{l}\text { 699-S38-E12B } \\
699-S 40-E 14 \\
699-S 41-E 11 \\
699-S 41-E 12 \\
699-S 41-E 13 A\end{array}$ & & $\begin{array}{l}46.80 \\
42.31 \\
43.26 \\
44.19 \\
51.17\end{array}$ & $\begin{array}{l}405.00 \\
402.85 \\
401.36 \\
401.93 \\
410.56\end{array}$ & $\begin{array}{l}358.20 \\
360.54 \\
358.10 \\
357.74 \\
359.39\end{array}$ \\
\hline
\end{tabular}


Appendix A: December 1993 Water Level Measurement Data (Sheet 22 of 22)

\begin{tabular}{c|c|c|c|c}
\hline \multirow{2}{*}{ We11 } & \multirow{2}{*}{ Notes } & \multirow{2}{*}{$\begin{array}{c}\text { Depth to } \\
\text { Water, ft }\end{array}$} & \multicolumn{2}{|c}{ Elevation, ft above ms 1 } \\
\cline { 3 - 5 } & & & Adjusted Casing & Water Leve1 \\
\hline 699-S41-E13B & & 50.69 & 410.10 & 359.41 \\
$699-S 41-$ E13C & & 51.56 & 410.67 & 359.11 \\
$699-S 43-E 12$ & & 48.69 & 405.60 & 356.91
\end{tabular}

Notes: $N=$ Well not included on map

$P=$ Well in confined aquifer, not included on map

$Q=$ Questionable data, not included on map 
WHC-EP-0394-8

\section{APPENDIX B}

WATER LEVEL DIFFERENCE, 200 AREAS 
Appendix B: Water Level Difference, 200 Areas, December 1988 to December 1993 (Sheet 1 of 5)

\begin{tabular}{|c|c|c|c|}
\hline Well & $\begin{array}{c}12 / 88 \\
\text { Water level }\end{array}$ & $\begin{array}{c}12 / 93 \\
\text { Water level }\end{array}$ & Change \\
\hline $\begin{array}{l}299-E 13-10 \\
299-E 17-1 \\
299-E 17-10 \\
299-E 17-14 \\
299-E 17-16\end{array}$ & $\begin{array}{l}405.85 \\
405.50 \\
406.08 \\
406.83 \\
405.87\end{array}$ & $\begin{array}{l}401.79 \\
401.57 \\
401.50 \\
401.44 \\
401.22\end{array}$ & $\begin{array}{l}-4.06 \\
-3.93 \\
-4.58 \\
-5.39 \\
-4.65\end{array}$ \\
\hline $\begin{array}{l}299-E 17-17 \\
299-E 17-18 \\
299-E 17-20 \\
299-E 17-5 \\
299-E 17-6\end{array}$ & $\begin{array}{l}404.57 \\
404.68 \\
405.74 \\
404.57 \\
405.95\end{array}$ & $\begin{array}{l}401.34 \\
401.36 \\
401.58 \\
400.95 \\
401.42\end{array}$ & $\begin{array}{l}-3.23 \\
-3.32 \\
-4.16 \\
-3.62 \\
-4.53\end{array}$ \\
\hline $\begin{array}{l}299-E 17-9 \\
299-E 18-1 \\
299-E 18-3 \\
299-E 23-1 \\
299-E 23-2\end{array}$ & $\begin{array}{l}405.42 \\
406.03 \\
406.02 \\
406.07 \\
405.99\end{array}$ & $\begin{array}{l}401.20 \\
401.89 \\
401.32 \\
401.53 \\
401.27\end{array}$ & $\begin{array}{l}-4.22 \\
-4.14 \\
-4.70 \\
-4.54 \\
-4.72\end{array}$ \\
\hline $\begin{array}{l}299-E 24-2 \\
299-E 24-7 \\
299-E 25-11 \\
299-E 25-19 \\
299-E 25-26\end{array}$ & $\begin{array}{l}405.71 \\
406.08 \\
406.23 \\
406.18 \\
408.05\end{array}$ & $\begin{array}{l}401.96 \\
401.29 \\
401.93 \\
401.91 \\
402.12\end{array}$ & $\begin{array}{l}-3.75 \\
-4.79 \\
-4.30 \\
-4.27 \\
-5.93\end{array}$ \\
\hline $\begin{array}{l}299-E 25-28 \\
299-E 25-32 P \\
299-E 25-34 \\
299-E 25-35 \\
299-E 27-10\end{array}$ & $\begin{array}{l}407.28 \\
407.53 \\
407.96 \\
406.39 \\
406.56\end{array}$ & $\begin{array}{l}402.14 \\
402.06 \\
402.04 \\
401.37 \\
401.96\end{array}$ & $\begin{array}{l}-5.14 \\
-5.47 \\
-5.92 \\
-5.02 \\
-4.60\end{array}$ \\
\hline $\begin{array}{l}299-E 27-8 \\
299-E 27-9 \\
299-E 28-26 \\
299-E 28-27 \\
299-E 32-1\end{array}$ & $\begin{array}{l}406.45 \\
406.01 \\
406.38 \\
405.54 \\
405.40\end{array}$ & $\begin{array}{l}401.64 \\
401.74 \\
401.45 \\
401.36 \\
401.57\end{array}$ & $\begin{array}{l}-4.81 \\
-4.27 \\
-4.93 \\
-4.18 \\
-3.83\end{array}$ \\
\hline $\begin{array}{l}299-E 32-2 \\
299-E 32-3 \\
299-E 32-4 \\
299-E 33-28 \\
299-E 33-29\end{array}$ & $\begin{array}{l}405.29 \\
405.36 \\
405.42 \\
405.67 \\
405.79\end{array}$ & $\begin{array}{l}401.20 \\
401.36 \\
401.19 \\
401.45 \\
401.45\end{array}$ & $\begin{array}{l}-4.09 \\
-4.00 \\
-4.23 \\
-4.22 \\
-4.34\end{array}$ \\
\hline
\end{tabular}


Appendix B: Water Level Difference, 200 Areas, December 1988 to December 1993 (Sheet 2 of 5)

\begin{tabular}{|c|c|c|c|}
\hline Well & $\begin{array}{l}12 / 88 \\
\text { Water level }\end{array}$ & $\begin{array}{c}12 / 93 \\
\text { Water level }\end{array}$ & Change \\
\hline $\begin{array}{l}299-E 33-30 \\
299-E 33-5 \\
299-E 34-2 \\
299-E 34-3 \\
299-E 34-5\end{array}$ & $\begin{array}{l}405.48 \\
405.39 \\
405.99 \\
406.53 \\
406.76\end{array}$ & $\begin{array}{l}401.42 \\
401.76 \\
401.63 \\
401.86 \\
402.78\end{array}$ & $\begin{array}{l}-4.06 \\
-3.63 \\
-4.36 \\
-4.67 \\
-3.98\end{array}$ \\
\hline $\begin{array}{l}299-E 34-6 \\
299-W 10-13 \\
299-W 10-14 \\
299-W 10-2 \\
299-W 10-8\end{array}$ & $\begin{array}{l}405.98 \\
466.34 \\
465.94 \\
466.76 \\
465.75\end{array}$ & $\begin{array}{l}401.67 \\
461.42 \\
461.06 \\
461.21 \\
460.83\end{array}$ & $\begin{array}{l}-4.31 \\
-4.92 \\
-4.88 \\
-5.55 \\
-4.92\end{array}$ \\
\hline $\begin{array}{l}299-W 11-10 \\
299-W 11-7 \\
299-W 15-12 \\
299-W 15-15 \\
299-W 15-18\end{array}$ & $\begin{array}{l}458.24 \\
465.01 \\
468.72 \\
468.94 \\
470.16\end{array}$ & $\begin{array}{l}453.04 \\
460.00 \\
462.63 \\
463.34 \\
463.96\end{array}$ & $\begin{array}{l}-5.20 \\
-5.01 \\
-6.09 \\
-5.60 \\
-6.20\end{array}$ \\
\hline $\begin{array}{l}299-W 15-5 \\
299-W 18-15 \\
299-W 18-22 \\
299-W 18-23 \\
299-W 18-24\end{array}$ & $\begin{array}{l}470.66 \\
471.56 \\
468.38 \\
469.27 \\
470.44\end{array}$ & $\begin{array}{l}464.99 \\
463.17 \\
462.91 \\
463.39 \\
464.11\end{array}$ & $\begin{array}{l}-5.67 \\
-8.39 \\
-5.47 \\
-5.88 \\
-6.33\end{array}$ \\
\hline $\begin{array}{l}299-W 19-15 \\
299-W 19-20 \\
299-W 19-21 \\
299-W 22-26 \\
299-W 23-11\end{array}$ & $\begin{array}{l}467.53 \\
461.53 \\
472.12 \\
464.59 \\
467.86\end{array}$ & $\begin{array}{l}460.16 \\
455.18 \\
464.65 \\
457.90 \\
460.18\end{array}$ & $\begin{array}{l}-7.37 \\
-6.35 \\
-7.47 \\
-6.69 \\
-7.68\end{array}$ \\
\hline $\begin{array}{l}299-W 23-4 \\
299-W 6-2 \\
299-W 7-1 \\
299-W 7-2 \\
299-W 7-3\end{array}$ & $\begin{array}{l}469.96 \\
461.62 \\
462.61 \\
460.89 \\
459.62\end{array}$ & $\begin{array}{l}460.96 \\
457.06 \\
457.71 \\
456.65 \\
455.60\end{array}$ & $\begin{array}{l}-9.00 \\
-4.56 \\
-4.90 \\
-4.24 \\
-4.02\end{array}$ \\
\hline $\begin{array}{l}299-W 7-4 \\
299-W 7-5 \\
299-W 7-6 \\
299-W 8-1 \\
299-W 9-1\end{array}$ & $\begin{array}{l}463.92 \\
460.10 \\
460.02 \\
462.32 \\
464.88\end{array}$ & $\begin{array}{l}458.48 \\
456.18 \\
456.05 \\
458.14 \\
460.50\end{array}$ & $\begin{array}{l}-5.44 \\
-3.92 \\
-3.97 \\
-4.18 \\
-4.38\end{array}$ \\
\hline
\end{tabular}


Appendix B: Water Level Difference, 200 Areas, December 1988 to December 1993 (Sheet 3 of 5)

\begin{tabular}{|c|c|c|c|}
\hline Well & $\begin{array}{c}12 / 88 \\
\text { Water Tevel }\end{array}$ & $\begin{array}{c}12 / 93 \\
\text { Water level }\end{array}$ & Change \\
\hline $\begin{array}{l}699-24-33 \\
699-24-34 B \\
699-24-35 \\
699-25-34 A \\
699-25-34 C\end{array}$ & $\begin{array}{l}404.94 \\
404.95 \\
404.83 \\
405.00 \\
405.46\end{array}$ & $\begin{array}{l}401.01 \\
401.14 \\
401.15 \\
401.08 \\
401.11\end{array}$ & $\begin{array}{l}-3.93 \\
-3.81 \\
-3.68 \\
-3.92 \\
-4.35\end{array}$ \\
\hline $\begin{array}{l}699-25-55 \\
699-25-70 \\
699-26-33 \\
699-26-34 \\
699-26-35 A\end{array}$ & $\begin{array}{l}413.50 \\
449.43 \\
405.14 \\
405.18 \\
405.17\end{array}$ & $\begin{array}{l}411.96 \\
445.86 \\
400.86 \\
401.16 \\
401.11\end{array}$ & $\begin{array}{l}-1.54 \\
-3.57 \\
-4.28 \\
-4.02 \\
-4.06\end{array}$ \\
\hline $\begin{array}{l}699-28-40 \\
699-28-52 A \\
699-29-78 \\
699-31-31 \\
699-32-43\end{array}$ & $\begin{array}{l}405.53 \\
406.79 \\
465.14 \\
405.02 \\
405.90\end{array}$ & $\begin{array}{l}401.39 \\
402.98 \\
459.30 \\
401.05 \\
401.70\end{array}$ & $\begin{array}{l}-4.14 \\
-3.81 \\
-5.84 \\
-3.97 \\
-4.20\end{array}$ \\
\hline $\begin{array}{l}699-32-62 \\
699-32-70 B \\
699-32-72 \\
699-32-77 \\
699-33-42\end{array}$ & $\begin{array}{l}429.41 \\
452.66 \\
455.08 \\
463.77 \\
406.00\end{array}$ & $\begin{array}{l}428.27 \\
448.80 \\
450.66 \\
457.93 \\
401.02\end{array}$ & $\begin{array}{l}-1.14 \\
-3.86 \\
-4.42 \\
-5.84 \\
-4.98\end{array}$ \\
\hline $\begin{array}{l}699-34-39 A \\
699-34-41 B \\
699-34-42 \\
699-34-51 \\
699-35-66\end{array}$ & $\begin{array}{l}405.89 \\
406.09 \\
406.03 \\
406.45 \\
440.03\end{array}$ & $\begin{array}{l}401.47 \\
401.79 \\
401.88 \\
401.97 \\
437.89\end{array}$ & $\begin{array}{l}-4.42 \\
-4.30 \\
-4.15 \\
-4.48 \\
-2.14\end{array}$ \\
\hline $\begin{array}{l}699-35-70 \\
699-35-78 A \\
699-36-61 A \\
699-37-82 A \\
699-38-65\end{array}$ & $\begin{array}{l}452.64 \\
468.36 \\
409.62 \\
468.61 \\
430.88\end{array}$ & $\begin{array}{l}448.78 \\
462.62 \\
406.50 \\
464.53 \\
428.92\end{array}$ & $\begin{array}{l}-3.86 \\
-5.74 \\
-3.12 \\
-4.08 \\
-1.96\end{array}$ \\
\hline $\begin{array}{l}699-38-70 \\
699-39-79 \\
699-40-33 A \\
699-40-62 \\
699-42-40 B\end{array}$ & $\begin{array}{l}454.60 \\
469.11 \\
411.37 \\
407.68 \\
423.41\end{array}$ & $\begin{array}{l}450.06 \\
463.23 \\
410.42 \\
404.61 \\
421.90\end{array}$ & $\begin{array}{r}-4.54 \\
-5.88 \\
-.95 \\
-3.07 \\
-1.51\end{array}$ \\
\hline
\end{tabular}


Appendix B: Water Level Difference, 200 Areas, December 1988 to December 1993 (Sheet 4 of 5)

\begin{tabular}{|c|c|c|c|}
\hline Well & $\begin{array}{c}12 / 88 \\
\text { Water level }\end{array}$ & $\begin{array}{c}12 / 93 \\
\text { Water level }\end{array}$ & Change \\
\hline $\begin{array}{l}699-42-42 B \\
699-43-42 J \\
699-43-43 \\
699-44-42 \\
699-44-64\end{array}$ & $\begin{array}{l}418.32 \\
420.59 \\
416.23 \\
422.07 \\
408.33\end{array}$ & $\begin{array}{l}417.18 \\
418.81 \\
414.97 \\
420.67 \\
405.84\end{array}$ & $\begin{array}{l}-1.14 \\
-1.78 \\
-1.26 \\
-1.40 \\
-2.49\end{array}$ \\
\hline $\begin{array}{l}699-45-42 \\
699-47-46 A \\
699-47-60 \\
699-48-71 \\
699-49-79\end{array}$ & $\begin{array}{l}419.16 \\
407.18 \\
405.06 \\
447.08 \\
457.90\end{array}$ & $\begin{array}{l}416.10 \\
403.15 \\
401.56 \\
444.19 \\
455.20\end{array}$ & $\begin{array}{l}-3.06 \\
-4.03 \\
-3.50 \\
-2.89 \\
-2.70\end{array}$ \\
\hline $\begin{array}{l}699-50-42 \\
699-50-85 \\
699-51-63 \\
699-51-75 \\
699-53-35\end{array}$ & $\begin{array}{l}411.59 \\
456.43 \\
406.83 \\
450.59 \\
397.71\end{array}$ & $\begin{array}{l}410.18 \\
454.69 \\
404.01 \\
448.40 \\
400.43\end{array}$ & $\begin{array}{r}-1.41 \\
-1.74 \\
-2.82 \\
-2.19 \\
2.72\end{array}$ \\
\hline $\begin{array}{l}699-53-47 B \\
699-53-48 A \\
699-53-55 B \\
699-53-55 C \\
699-54-48\end{array}$ & $\begin{array}{l}406.44 \\
404.62 \\
404.01 \\
404.26 \\
404.17\end{array}$ & $\begin{array}{l}405.41 \\
403.05 \\
400.78 \\
401.06 \\
400.96\end{array}$ & $\begin{array}{l}-1.03 \\
-1.57 \\
-3.23 \\
-3.20 \\
-3.21\end{array}$ \\
\hline $\begin{array}{l}699-55-40 \\
699-55-50 C \\
699-55-70 \\
699-55-76 \\
699-57-83 A\end{array}$ & $\begin{array}{l}409.78 \\
404.18 \\
432.73 \\
444.90 \\
432.95\end{array}$ & $\begin{array}{l}408.67 \\
400.95 \\
431.47 \\
443.50 \\
432.45\end{array}$ & $\begin{array}{l}-1.11 \\
-3.23 \\
-1.26 \\
-1.40 \\
-.50\end{array}$ \\
\hline $\begin{array}{l}699-59-32 \\
699-59-58 \\
699-59-80 B \\
699-60-32 \\
699-60-60\end{array}$ & $\begin{array}{l}361.87 \\
403.37 \\
427.78 \\
361.70 \\
403.99\end{array}$ & $\begin{array}{l}361.34 \\
400.76 \\
429.55 \\
361.19 \\
400.71\end{array}$ & $\begin{array}{r}-.53 \\
-2.61 \\
1.77 \\
-.51 \\
-3.28\end{array}$ \\
\hline $\begin{array}{l}699-61-37 \\
699-51-41 \\
699-61-62 \\
699-61-66 \\
699-62-31\end{array}$ & $\begin{array}{l}382.15 \\
396.40 \\
402.90 \\
402.78 \\
361.62\end{array}$ & $\begin{array}{l}381.21 \\
395.47 \\
400.80 \\
399.99 \\
361.14\end{array}$ & $\begin{array}{r}-.94 \\
-.93 \\
-2.10 \\
-2.79 \\
-.48\end{array}$ \\
\hline
\end{tabular}


Appendix B: Water Level Difference, 200 Areas, December 1988 to December 1993 (Sheet 5 of 5)

\begin{tabular}{l|c|c|c}
\hline \multicolumn{1}{c|}{ We11 } & $\begin{array}{c}12 / 88 \\
\text { Water level }\end{array}$ & $\begin{array}{c}12 / 93 \\
\text { Water level }\end{array}$ & Change \\
\hline $699-62-43 A$ & 396.74 & 395.73 & -1.01 \\
$699-63-51$ & 400.71 & 398.73 & -1.98 \\
$699-63-58$ & 402.28 & 399.67 & -2.61 \\
$699-64-62$ & 401.90 & 399.41 & -2.49 \\
$699-65-50$ & 400.70 & 398.70 & -2.00 \\
$699-65-59 A$ & 401.79 & 399.40 & -2.39 \\
$699-65-72$ & 399.36 & 397.41 & -1.95
\end{tabular}


WHC-EP-394-8

\section{DISTRIBUTION}

Number of copies

offsite

1

\section{U.S. Geological Survey}

B. W. Drost

1201 Pacific Ave. Suite 600

Tacoma, WA 98402

Onsite

3

U.S. Department of Energy. Richland Operations office

M. J. Furman

R3-80

A. J. Lassila

S7 -52

K. M. Thompson

A5-19

2

Washington State Department of Ecology

S. Leja

$\mathrm{N} 1-08$

M. A. Selby

$\mathrm{N} 1-08$

2

U.S. Environmental Protection Agency

D. R. Sherwood (2)

B5-01

13

Pacific Northwest Laboratory

M. P. Bergeron

K6-77

J. V. Borghese

K6-96

R. W. Bryce

M. A. Chamness

K6-96

K6-84

M. D. Freshley

$\mathrm{K} 6-77$

S. M. Goodwin

K6-84

E. L. Hilty

K6-96

G. V. Last

K6-84

S. P. Luttrell

K6-96

D. R. Newcomer

K6-96

R. M. Smith

F. A. Spane

K6-96

K6-96

W. D. Webber

K6-96

73

Westinghouse Hanford Company
M. R. Adams
D. J. Alexander
D. B. Barnett
M. A. Buckmaster

H6-01

H6-06

H6-06

H6-03 
Westinghouse Hanford Company (cont)

J. A. Caggiano

H6-06

L. B. Collard

H6-01

J. D. Davis

L. P. Diediker

H6-01

J. J. Dorian

T1-30

G. L. Dunford

H6-30

R. S. Edrington

$\mathrm{R} 1-51$

D. B. Erb

H6-06

J. W. Fassett

H6-03

K. R. Fecht

H6-06

M. A. Frank

H6-06

B. H. Ford

H6-03

M. G. Gardner

M. J. Hartman (5)

H6-06

N3-06

F. D. Hodges

H6-06

D. G. Horton

H6-06

R. L. Jackson

H6-06

V. G. Johnson

H6-06

W. L. Johnson

H6-06

W. A. Jordan

H6-04

G. L. Kasza (5)

H6-06

A. J. Knepp

H6-06

M. J. Lauterbach

H6-06

A. G. Law

H6-01

J. W. Lindberg

H6-06

K. A. Lindsey

H6-06

A. H. Lu

C. J. Lynch

H6-06

HO-36

W. J. McMahon

H6-01

R. B. Mercer

H6-06

R. D. Miller

H6-06

D. J. Moak

H6-06

S. E. Myers

N3-05

N3-06

S. M. Narbutovskih

H6-06

R. S. Paulina

R3-45

R. E. Peterson

H6-06

S. M. Price

H6-23

W. H. Price

N3-05

R. F. Raidl

H6-06

S. P. Reidel

H6-06

K. D. Reynolds

H6-06

V. J. Rohay

A. L. Schatz

H6-06

J. S. Schmid

N3-05

J. A. Serkowski

H6-06

K. R. Simpson

H6-06

L. C. Swanson

H6-06

M. D. Sweeney

H6-06

K. J. Swett

H6-06

R. R. Thompson

H6-06

E. C. Thornton

$\mathrm{H} 6-32$

S. J. Trent

H6-06

H6-06 
WHC-EP-394-8

Westinghouse Hanford Company (cont)

D. K. Tyler

H6-06

P. J. Valcich

H6-06

D. C. Weekes

C. D. Wittreich

H6-06

Central Files (2)

H6-03

EPIC (2)

Public Reading Room

L8-04

H6-08

Al-65 

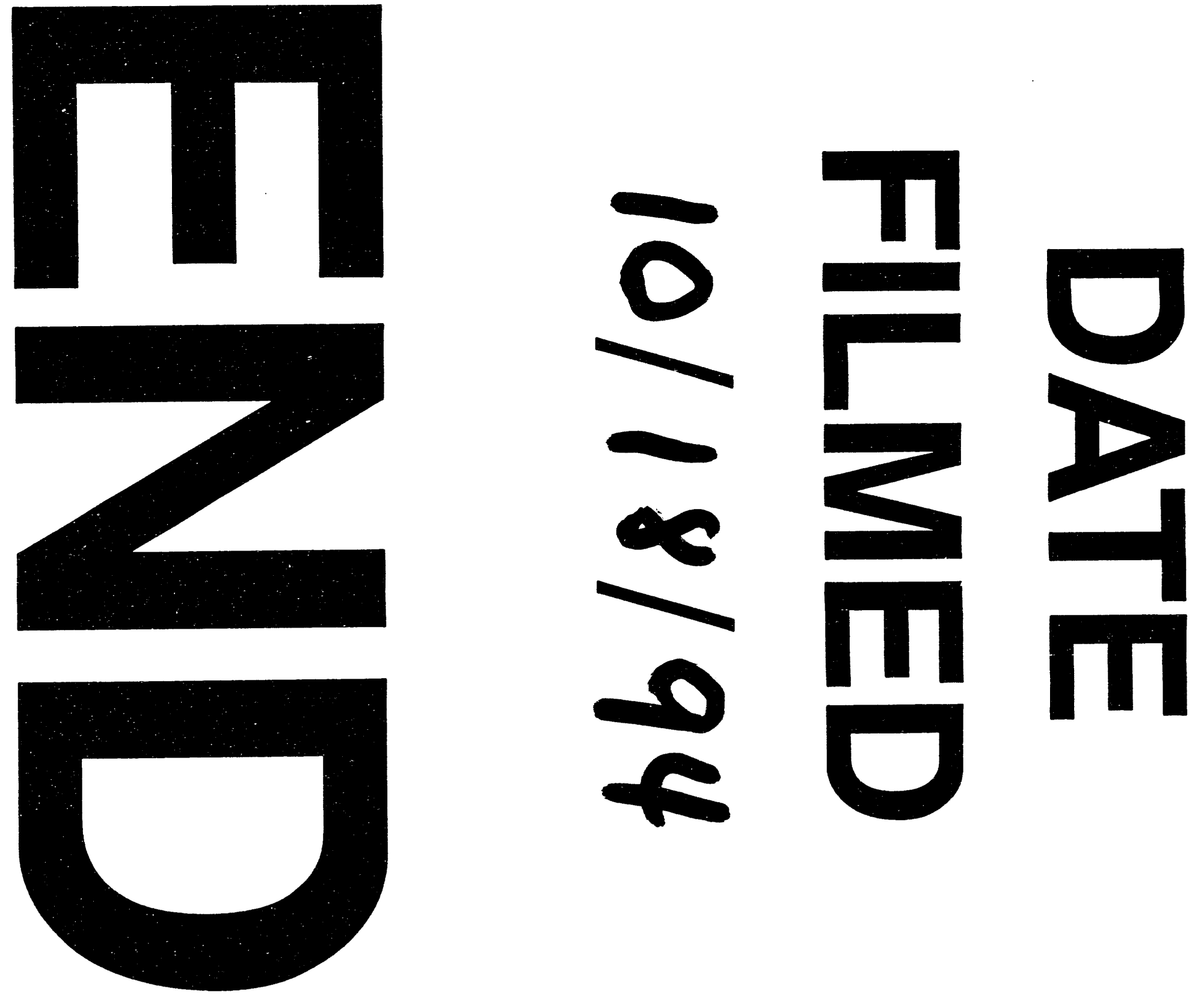
